\title{
BREAST IMMOBILIZATION DEVICE OPTIMIZATION FOR DUAL MODALITY TOMOSYNTHESIS
}

\author{
A thesis \\ presented to \\ the faculty of the School of Engineering and Applied Science \\ University of Virginia
}

\author{
In partial fulfillment \\ of the requirements for the degree \\ Master of Science in Biomedical Engineering
}

By

Kelly Lynn Klanian

December 2012 
APPROVAL SHEET

The thesis

is submitted in partial fulfillment of the requirements

for the degree of

Master of Science

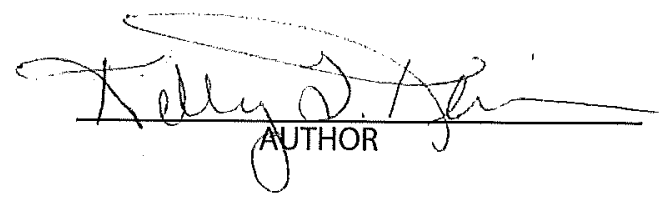

The thesis has been read and approved by the examining committee:

Dr. Mark Williams

\begin{tabular}{c}
\hline Advisor \\
Dr. Jason Papin \\
\hline Dr. Robert Ribando \\
\hline
\end{tabular}

Accepted for the School of Engineering and Applied Science:

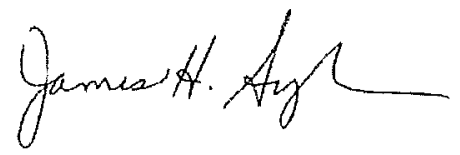

Dean, School of Engineering and Applied Science

December

2012 


\begin{abstract}
Conventional x-ray mammography continues to be the mainstay for breast cancer screening. However, traditional mammography has a multitude of limitations, including low sensitivity for the detection of early malignancies and high false positive rates. Breast compression using a flat compression paddle is commonly used to immobilize the breast as 2-D $\mathrm{X}$-ray images are acquired during a clinical scan. Although strong compression is used to flatten the breast and thus reduce the amount of superimposed tissue that can mask tumors in the twodimensional image, small masses can still be occult, especially in radio-dense breasts.
\end{abstract}

These challenges have motivated researchers to explore other imaging modalities beyond conventional x-ray mammography to aid in the fight against breast cancer. For example, our dual modality tomosynthesis (DMT) scanner performs both x-ray breast tomosynthesis (XBT) and molecular imaging breast tomosynthesis (MBIT) with the breast in a single configuration in order to assure that accurate co-registration can be obtained between the two resulting 3-D image sets. Both XBT and MBIT use a tomosynthesis image acquisition approach, in which multiple views of the breast are obtained over a range of viewing angles. XBT provides anatomical information and can reliably resolve objects on a sub-millimeter scale. MBIT provides functional information through the use of an intravenously injected tracer tagged with a radioisotope and has a spatial resolution on the order of 2.5 to $5.0 \mathrm{~mm}$. Standard, flat paddle compression has been used to immobilize the breast during pilot DMT studies. The 3-D nature of the tomosynthesis image reduces the problem of tumor masking since thin slices from the reconstructed volume can be displayed. Thus, some of the motivation for vigorous breast 
compression is eliminated. This, in part, has motivated our investigation of alternative strategies for immobilizing the breast during DMT scanning.

The overall goal of this project was to develop an innovative breast immobilization device that can be integrated into the DMT scanner to improve image quality as well as patient comfort. Our hypothesis was that MBIT image quality could be improved by reducing the radial distance between the breast itself and the gamma camera at large viewing angles (away from the direction of compression). In addition, we hypothesized that compressing the breast in a more natural configuration would increase the level of patient comfort during a scan. Preliminary experimental studies showed that our optimized breast immobilization device is capable of improving both image contrast and signal-to-noise ratio (SNR) as compared to standard, flat paddle compression. Furthermore, qualitative assessments were made with respect to patient comfort and noticeable improvements were observed with one exception. The single exception was that when evaluating the prototype immobilization device, women with large breasts expressed discomfort because the prototype was not wide enough to fully contain all of their breast tissue. Accordingly, we are in the process of fabricating similar immobilization devices with a range of sizes to be evaluated for their ability to comfortably accommodate a broad range of breast sizes, while still providing improved image contrast and SNR. 


\section{ACKNOWLEDEGMENTS}

First and foremost, I would like to acknowledge and thank all those who have supported me and offered constructive suggestions during the development of this research work. I am particularly grateful for the professional guidance extended to me by my advisor, Dr. Mark Williams. I wish to recognize the help provided by those who work tirelessly at the University of Virginia Breast Care Center and the Physics Machine Shop. In addition, assistance provided to me by my colleagues and friends was greatly appreciated. Lastly, I would like to thank my family for their love, support, patience and understanding throughout the duration of my studies. 


\section{TABLE OF CONTENTS}

$\begin{array}{ll}\text { Abstract } & \text { i }\end{array}$

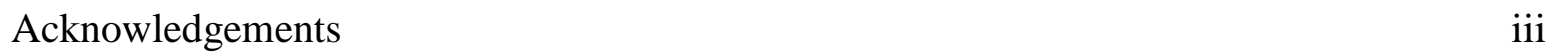

Table of Contents $\quad$ iv

List of Figures $\quad$ vi

List of Tables $\quad$ viii

$\begin{array}{ll}\text { List of Abbreviations } & \text { ix }\end{array}$

$\begin{array}{ll}\text { Chapter 1: Introduction } & 1\end{array}$

1.1 Current Practice: X-ray Mammography 2

1.2 Alternative Approaches to X-ray Mammography 3

$\begin{array}{ll}\text { 1.2.1 Breast Ultrasound (US) } & 4\end{array}$

1.2.2 Magnetic Resonance Imaging (MRI) 4

1.2.3 X-ray Breast Tomosynthesis (XBT) 5

$\begin{array}{ll}\text { 1.2.4 Nuclear Medicine Imaging (NM) } & 6\end{array}$

1.2.5 Diffuse Optical Tomography (DOT) 6

1.2.6 Dual Modality Tomosynthesis (DMT) 7

1.3 Breast Immobilization Device Design Limitations 9

Chapter 2: Breast Immobilization Device Optimization $\quad 11$

2.1 Design Evolution and Material Selection 12

2.2 Curvature Quantification 16

$\begin{array}{ll}2.3 \text { Truncation Calculations } & 19\end{array}$

2.4 Detailed Device Description and Fabrication 24

Chapter 3: Gelatin Breast Phantom and Gamma Camera Trajectory Studies 27

3.1 Study Design 28

3.1.1 Experimental Set-Up 28 
3.1.3 Trajectory Calculations 32

3.2 Reconstruction Algorithm Description 37

$\begin{array}{ll}3.3 \text { Results } & 38\end{array}$

3.4 Discussion

Chapter 4: Prototype Evaluation and Redesign 44

4.1 Optimized Breast Immobilization Device Design Limitations 47

$\begin{array}{ll}4.2 \text { Support System Limitations } & 48\end{array}$

4.3 Optimized Breast Immobilization Device Alternative Design 49

4.4 Support System Alternative Design $\quad 52$

Chapter 5: DMT Scanner Optimization $\quad 55$

5.1 Aluminum Arm Deficiencies $\quad 56$

5.2 Aluminum Arm Modifications $\quad 57$

5.3 X-ray Detector Support Deficiencies $\quad 57$

5.4 X-ray Detector Support Modifications $\quad 58$

Chapter 6: Summary, Conclusions, and Future Direction $\quad 61$

$\begin{array}{ll}6.1 \text { Summary } & 61\end{array}$

$\begin{array}{ll}6.2 \text { Conclusions } & 62\end{array}$

6.3 Future Direction $\quad 63$

Appendix A: Elliptical coordinates of optimized immobilization device 64

Appendix B: Optimized immobilization device truncation point calculations 74

Appendix C: Optimized immobilization device \& support drawings 76

Appendix D: Gamma camera trajectory coordinates 81

Appendix E: Image quality analysis files (Mat lab source code \& Excel files) 86

Appendix F: Enlarged optimized immobilization device \& support drawings 96

Appendix G: X-ray detector hinge \& lead brick weight sub-assembly drawings 106

$\begin{array}{ll}\text { References } & 115\end{array}$ 


\section{LIST OF FIGURES}

Figure 1-1: X-ray Mammogram: Standard Compression Illustration

Figure 1-2: $\quad$ Drawing of the Dual Modality Tomosynthesis Scanner

Figure 1-3: $\quad$ Photograph of the Dual Modality Tomosynthesis Scanner

Figure 2-1: $\quad$ Bolx I \& II Bolus Material (JRT Associates)

Figure 2-2: $\quad$ Breast Cancer Incidence

Figure 2-3: $\quad$ Breast Anatomy

Figure 2-4: Photograph of "tongue-and-groove" alternative design

Figure 2-5: "Tongue-and-groove" tomographic image slice

Figure 2-6: Breast Pump

Figure 2-7: $\quad$ Molecular Structure of Polycarbonate

Figure 2-8: Compressive Strength of Polycarbonate

Figure 2-9: Photograph of Experimental Set-up to determine semi-major and semi-minor axis of an ellipse defining the breast immobilization device

Figure 2-10: Schematic of an ellipse used to derive the $\mathrm{x}$ - and $\mathrm{y}$-coordinates of the breast immobilization device

Figure 2-11: Illustration of truncation locations and area within the truncated breast immobilization device

Figure 2-12: Trigonometric Substitution Triangle

Figure 2-13: Photograph of Optimized Breast Immobilization Device

Figure 3-1: $\quad$ Photograph of Experimental Set-up

Figure 3-2: $\quad$ K-max GUI

Figure 3-3: "Flat Paddle" Trajectory Schematic

Figure 3-4: Optimized Breast Immobilization Device Trajectory Schematic

Figure 3-5: In-plane Slice Image Used to Determine Lesion Intensity (parallel to the x-y plane of the gelatin breast phantom)

Figure 3-6: In-plane Slice Image Used to Determine Background Intensity (parallel to the x-y plane of the gelatin breast phantom) 
Figure 3-7: Lesion Contrast. Error Bars indicate standard deviation of 5 repeated trials of the experiment

Figure 3-8: $\quad$ SNR. Error Bars indicate standard deviation of 5 repeated trials of the experiment

Figure 3-9: Box and whiskers plot comparing contrast (shallow lesion)

Figure 3-10: Box and whiskers plot comparing contrast (deep lesion)

Figure 3-11: Box and whiskers plot comparing SNR (shallow lesion)

Figure 3-12: $\quad$ Box and whiskers plot comparing SNR (deep lesion)

Figure 4-1: $\quad$ Pregnancy bell cast kit

Figure 4-2: $\quad$ Mold of breast compressed with optimized immobilization device

Figure 4-3: Cross-sectional area of truncated optimized breast immobilization device

Figure 4-4: $\quad$ Example acquired image of a large breast

Figure 5-1: $\quad$ Photograph of aluminum arm

Figure 5-2: $\quad$ Photograph of $\mathrm{x}$-ray detector housing

Figure 5-3: $\quad$ Photograph of x-ray detector \& patient arm collision 


\section{LIST OF TABLES}

Table 1-1: $\quad$ Incidence and Mortality Rates in the United States

Table 3-1: $\quad$ Spreadsheet calculating background and lesion activity

Table 3-2: $\quad$ Spreadsheet calculating image acquisition time

Table 3-3: $\quad$ P-values associated with contrast and SNR according to lesion depth 


\section{LIST OF ABBREVIATIONS}

${ }^{99 \mathrm{~m}} \mathrm{Tc}=$ technetium $99 \mathrm{~m}$

$\mathrm{Al}=$ aluminum

ACR = American College of Radiology

ANOVA $=$ analysis of variance

$\mathrm{AOR}=$ axis-of-rotation

$\mathrm{Be}=$ beryllium

$\mathrm{Ci}=$ curie

CMOS $=$ complementary metal-oxide-semiconductor

$\mathrm{CsI}=$ cesium iodide

DCIS $=$ ductal carcinoma in situ

DMT = dual-modality tomographic

DOI $=$ diffuse optical imaging

FOV $=$ field-of-view

FWHM = full-width-at-half-maximum

$\mathrm{kVp}=$ kilovolt peak

MBIT = molecular imaging breast tomosynthesis

$\mathrm{ML}=$ medial-lateral

$\mathrm{MLO}=$ medial-lateral oblique

MRI = magnetic resonance imaging

$\mathrm{NIH}=$ national institute of health

$\mathrm{NM}=$ nuclear medicine imaging

$\mathrm{PC}=$ polycarbonate

$\mathrm{PMT}=$ photomultiplier tube

PSPMT $=$ position sensitive photomultiplier tube 
$\mathrm{QQ}=$ quantile-quantile

$\mathrm{Rh}=$ rhodium

ROI $=$ region of interest

SBI $=$ Society of Breast Imaging

SNR = signal-to-noise ratio

US $=$ ultrasound

$\mathrm{W}=$ tungsten

$\mathrm{XBT}=\mathrm{x}$-ray breast tomosynthesis 
CHAPTER 1

INTRODUCTION

Apart from non-melanoma skin cancer, breast cancer is the most common cancer among women in the United States and is also one of the leading causes of cancer death among women of all races around the world [10]. Breast cancer incidence rates vary depending on a number of different factors including gender, age, race, and ethnicity [10]. The Susan G. Komen foundation recently estimated that the number of new cases of breast cancer (both invasive and non-invasive) among U.S. women in 2012 will total nearly 300,000, while approximately 40,000 deaths will be attributed to the disease [27]. Gender specific incidence and mortality rates in the United States are reported in Table 1-1. Risk factors abound for breast cancer, though the two most important are gender and age [27]. 


\begin{tabular}{|l|c|c|}
\hline & Men & Women \\
\hline $\begin{array}{l}\text { Incidence (new } \\
\text { cases) }\end{array}$ & 1.2 per 100,000 & 125.7 per 100,000 \\
\hline $\begin{array}{l}\text { Mortality } \\
\text { (deaths) }\end{array}$ & 0.3 per 100,000 & 22.2 per 100,000 \\
\hline
\end{tabular}

\section{Table 1-1: Incidence and Mortality Rates in the United States [10]}

Most health care professionals would agree that the best defense against breast cancer is early detection. As such, the American College of Radiology (ACR) and the Society of Breast Imaging (SBI) recently released recommendations on breast cancer screening in The Journal of the American College of Radiology. These recommendations suggest that those women who are at average risk for breast cancer should start annual mammograms at age 40 , while those who have a higher risk should start breast cancer screening at least by age 30 [5].

\subsection{Current Practice: X-ray Mammography}

X-ray mammography is the traditionally accepted modality for breast cancer screening. During a mammogram, a woman's breast is positioned by a radiation technologist and exposed to ionizing radiation to produce an image. The breast is immobilized with a flat compression paddle as illustrated in Figure 1-1. On average, twenty pounds force is typically applied to compress a woman's breast during a clinical exam. Most women who have experienced a clinical mammogram express angst and discomfort. In addition, mammography has reportedly missed $10-15 \%$ of breast cancers [11] and contributes to false positive readings. As a result, patients experience a sense of heightened anxiety and apprehension. Additional diagnostic tests 
are oftentimes required to definitively determine disease presence, which also augments inflationary health care costs. Consequently, researchers and clinicians alike are expressing interest in the use of alternative imaging modalities in conjunction with mammography to combat these ever persistent problems.

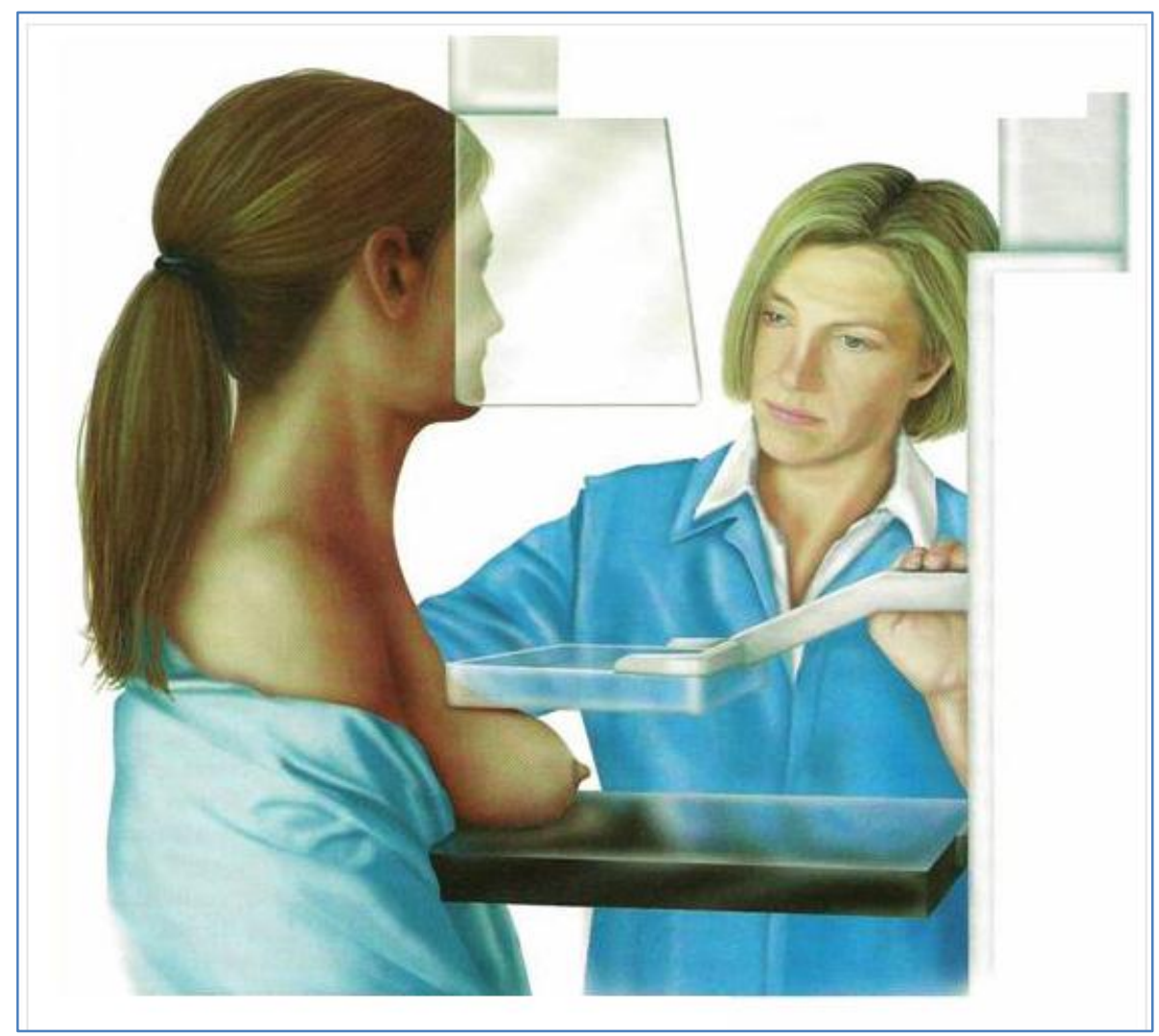

Figure 1-1: X-ray Mammogram: Standard Compression Illustration [14]

\subsection{Alternative Approaches to X-ray Mammography}

Alternative imaging modalities currently used in clinical practice include: Breast Ultrasound (US), Magnetic resonance imaging (MRI) of the breast, X-ray Breast tomosynthesis (XBT), and Nuclear Medicine Imaging (NM). Those alternatives currently under development include: Diffuse Optical Tomography (DOT) and Dual Modality Tomosynthesis (DMT). A 
brief description as well as the advantages and disadvantages of each of these alternatives will be discussed in the paragraphs that follow.

\subsubsection{Breast Ultrasound (US)}

Ultrasound imaging also referred to as ultrasound scanning or sonography, involves exposing the breast to high-frequency sound waves to produce a picture of the internal structure [9]. An ultrasound image is acquired by sweeping a transducer across the breast after a clear gel is applied to the skin [9]. Ultrasound imaging is oftentimes touted as an adjunct screening tool for women with very dense breasts or silicone breast implants [9]. It is also used to screen women who may be pregnant and do not want to be exposed to x-rays [9]. However, it has been reported that the sensitivity of ultrasound alone is $39.5 \%$, while the sensitivity of mammography and ultrasound together is $48.8 \%$ [17]. Breast ultrasound screening has not been widely accepted because of issues related to reproducibility, high-false-positive rates, operator dependencies of the examination, and inability to image most DCIS cases [18].

\subsubsection{Magnetic Resonance Imaging (MRI)}

A powerful magnetic field, radio waves and a computer are used in MRI to produce detailed pictures of the breast [8]. Hundreds of images are generated during each exam and in most cases, the imaging physician requests a contrast agent (dye) to improve the quality of the images [8]. An image of the breast is acquired by having the patient lay in the prone position on a table with a special device called a coil which is also used to improve image quality [8]. MRI is performed, in addition to annual mammography, for "women with $>20 \%$ lifetime risk for the development of breast cancer" [18]. It has been reported that the sensitivity of MRI is $90.7 \%$, while the sensitivity of mammography and MRI together is $93 \%$ [17]. As emphasized by the 
ACS, screening with MRI is "inappropriate for women at $<15 \%$ lifetime risk for breast cancer" [18]. The ACS also suggests that breast MRI is not meant to replace mammography because there are cases, particularly of ductal carcinoma in situ (DCIS), which are only detectable by mammography alone [18]. Furthermore, the addition of breast MRI to the screening algorithm for women at greatest risk is considerably expensive [18]. As such, it is important to consider its use on a case-by-case basis.

\subsubsection{X-ray Breast Tomosynthesis (XBT)}

$\mathrm{X}$-ray tomosynthesis acquisition is similar to conventional mammography with regard to breast positioning and compression, but differs in that the X-ray tube takes multiple low-dose exposures as it moves through a limited (e.g., 30) arc of motion [23]. The resulting projection images are reconstructed into a 3D tomographic image of the breast, sectioned in the orientation of acquisition [23]. XBT is the most recent modality to be implemented in the clinical environment, and experts agree that it does provide some significant advantages over conventional mammography. These advantages include "permitted depth localization, improved conspicuity of structures by removing the visual clutter associated with overlying anatomy, and improved local structure contrast by restricting the overall image dynamic range to that of a single slice" [12]. It has been reported that the use of tomosynthesis as an adjunct to digital screening mammography leads to a decrease in the recall rate by nearly half [23]; however, these results must be validated as XBT becomes more widely accepted by clinicians and researchers alike. 


\subsubsection{Nuclear Medicine Imaging (NM)}

Unlike traditional $\mathrm{x}$-ray examinations, nuclear medicine uses radiation that is emitted from a patient's body after a radioactive material is introduced [15]. This radiation is in the form of gamma rays, which are a lot like x-rays, but have a shorter wavelength [15]. Gamma ray signals are measured by a gamma camera which consists of a crystal array (called scintillation crystals) [15]. The crystals detect the emitted photon and convert it into light, which is then transformed into an electric signal and digitized (converted into a computer signal) to reconstruct an image that can be easily interpreted [15]. One of the unique things that nuclear medicine tests add in terms of disease detection is extreme sensitivity to abnormalities in an organ's function [25]. However, nuclear medicine imaging cannot yet provide spatial resolution on the order of microns, as is commonly observed with conventional x-ray imaging techniques.

\subsubsection{Diffuse Optical Tomography (DOT)}

Diffuse optical tomography can be used to screen for breast cancer and should be able to provide quantitative images of tissue absorption and diffusion coefficient maps [22]. As suggested by its name, diffuse optical tomography uses multiple projection images in conjunction with a reconstruction algorithm to improve spatial resolution over conventional diaphanography [22]. The optical source of light for the imaging system is a diode laser [22]. The light signal is delivered to and from the tissue via fiber optic cables, and light detection is accomplished with a single photomultiplier tube (PMT) [22]. Both a change in blood volume and a change in blood oxygenation can alter the light amplitude signal [3]. This method is currently being explored as a breast screening tool because of its ability to reveal the aforementioned changes that are specific to early stage cancer [3]. DOT is an attractive imaging method because 
it is inexpensive, portable, and fast [3]. That being said, because it has low spatial resolution, DOT could benefit from structural guidance by combining it with other higher spatial resolution imaging tools [3].

\subsubsection{Dual Modality Tomosynthesis (DMT)}

Note: significant portions of Section 1.2.6 were taken with permission from reference [16]

Dual modality tomosynthesis differs significantly from the clinical and developmental imaging techniques mentioned herein. The Dual Modality Tomosynthesis Scanner, as depicted in Figures 1-2 and 1-3, acquires both x-ray and gamma-ray projection images across a limited angle range. The $\mathrm{x}$-ray tube, $\mathrm{x}$-ray detector, and gamma camera rotate around a central axis of rotation (AOR) to perform both XBT and MBIT with the breast in a single configuration. This ensures that the resulting 3-D image sets can be properly co-registered. The gantry itself was built by Dexela Ltd., a PerkinElmer Company (London, England). A Dexela 2923MAM flat panel complementary metal-oxide-semiconductor (CMOS) x-ray detector was mounted to the gantry arm, as was a gamma camera, provided by Jefferson Labs. Angular rotation of the gantry arm is controlled by a software program written by Dexela.

In addition to the CMOS detector, the x-ray system is comprised of a high output, oilcooled tungsten (W) target X-ray tube (Varian model RAD 70) with a nominal focal spot size of $0.3 \mathrm{~mm}$ in the left-right direction and $0.43 \mathrm{~mm}$ in the anode-cathode direction. The system also contains a $0.76 \mathrm{~mm}$ beryllium $(\mathrm{Be})$ window and $0.050 \mathrm{~mm}$ rhodium $(\mathrm{Rh})$ filter. The array size of the x-ray detector is $3072 \times 3888$, with a pitch of $75 \mu \mathrm{m}$ in $1 \times 1$ binning mode. The active area of the detector is 230 x $290 \mathrm{~mm}$. Its high frame rate (up to 26 frames/sec) and its low noise properties make it ideal for tomosynthesis applications. 
The gamma camera consists of a 3 x 4 array of Hamamatsu H8500 position sensitive photomultiplier tubes (PSPMTs) that are optically coupled to a NaI(TI) crystal array with a crystal pitch of $2.2 \mathrm{~mm}$ and a crystal thickness of $6 \mathrm{~mm}$. The camera is also equipped with a lead parallel hole collimator that has an associated efficiency of $2.34 \times 10^{-4}$, while the acquisition is controlled by software written in K-max (Sparrow Corporation, Port Orange, FL). The camera, as previously mentioned, is mounted to the gantry arm via a motor controlled Velmex bi-slide linear translation stage system which permits the gamma camera to move up and down using an analog joystick. The gamma camera is moved manually along two slider rails towards the patient in the posterior direction to acquire gamma images after $\mathrm{x}$-ray image acquisition has been completed.

Though there are marked differences between this system and most of those previously mentioned one obvious similarity is worth noting. Flat paddle compression is the traditionally accepted format for rendering the breast immobile during image acquisition. Much like its clinical counterparts, the DMT breast immobilization device is comprised of a flat breast support and compression paddle, where the radial distance between the compression paddle and the AOR is adjustable. Polycarbonate (PC) is used to manufacture compression paddles because it exhibits both strength and ductility, important properties when considering patient safety. However, the flat nature of the current technology does present some significant limitations, especially for tomographic applications. 


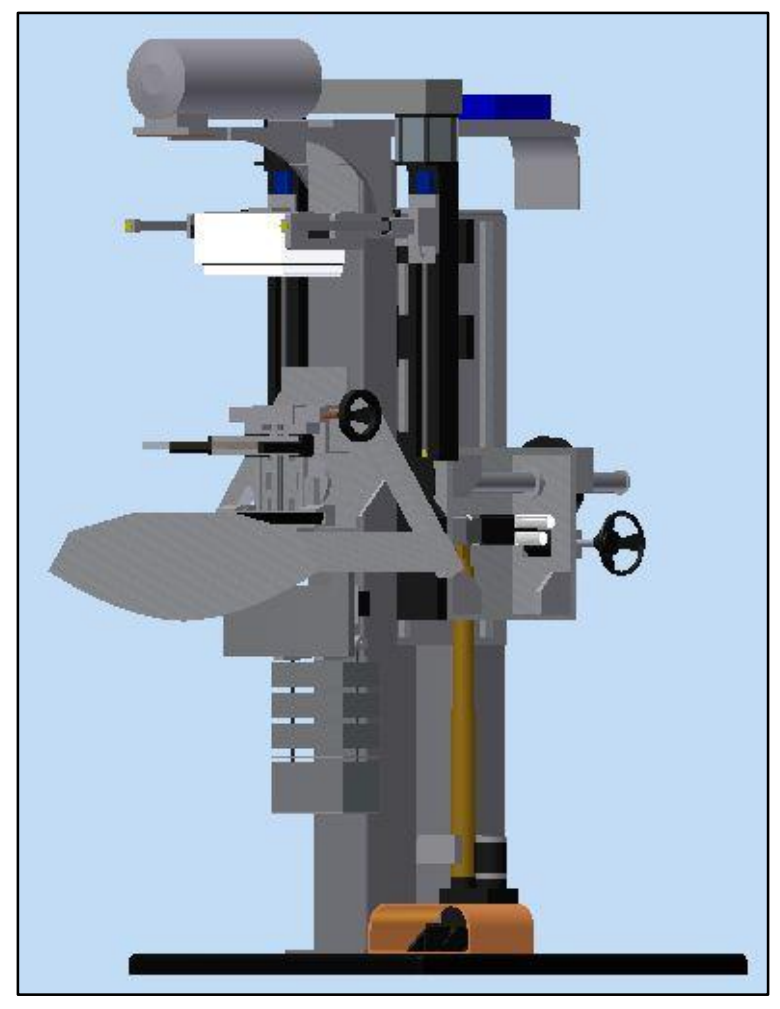

Figure 1-2: Drawing of the Dual Modality Tomosynthesis Scanner

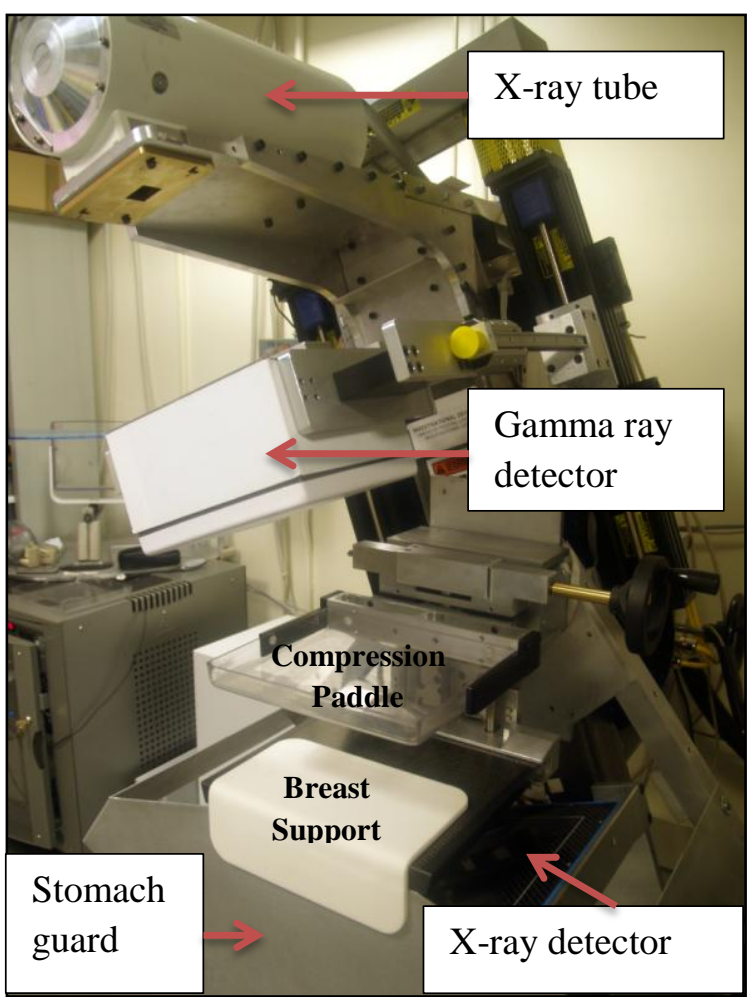

Figure 1-3: Photograph of the Dual Modality Tomosynthesis Scanner

\subsection{Breast Immobilization Device Design Limitations}

Flat paddle compression is primarily used to hold the breast firmly in place during image acquisition. During compression, superimposed normal breast tissue obscures malignancies, making them difficult to detect. This is especially true for women with radio dense breasts, as healthy dense tissue and diseased tissue have a similar appearance in the acquired structural image. Additionally, flat paddle compression restricts the viewing angle range for imaging system positioning. Acquiring images over a broader range of viewing angles would result in an image set that more accurately depicts the breast structure itself and any cancerous lesions that may be present. Most tomographic imaging systems operate within a very limited angular range 
due to the restrictive nature of the hardware and the associated risk of system component collisions. In addition, the flat nature of the compression paddle does not permit close proximity between the breast and the imaging system, particularly at large angles away from the zero degree view. As the radial distance between the breast and the imaging system increases, the quality of the image itself decreases and it becomes increasingly difficult to accurately resolve malignancies, especially those in the early stages of disease progression. All of these design limitations have motivated us to optimize the breast immobilization device to improve image quality and provide our patients with a more pleasant imaging experience. 


\section{BREAST IMMOBILIZATION DEVICE OPTIMIZATION}

Having investigated a number of different alternatives to flat paddle compression, including the use of flexible materials and breast suctioning, we believe that the best way to immobilize the breast and achieve our technical goals is to implement a design that consists of a rigid yet curved compression paddle and breast support. Both the compression paddle and support are mounted on a mechanical positioning system that permits their separation to be adjusted in order to accommodate a variety of breast thicknesses. The technical attributes associated with the breast immobilization device include secure breast placement, proper shape for imaging the full breast, and contoured design to permit close access to the breast by the imaging device, while simultaneously maximizing patient comfort. The evolution of the design was driven by the need to create enough compressive force to prevent the breast from moving during the scan and ensure that the resulting image contains all of the necessary breast tissue. In 
addition, both the compression paddle and breast support are crafted from a low-attenuation material (polycarbonate), thereby permitting minimal reduction in the signal intensity.

\subsection{Design Evolution and Material Selection}

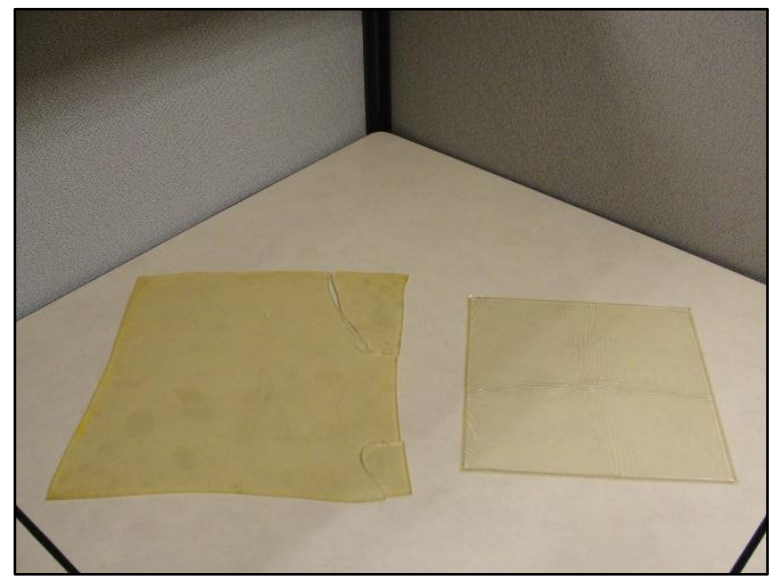

Figure 2-1: Bolx I \& II Bolus Material (JRT Associates)
A multitude of flexible materials were explored as the design process ensued, and of those considered, Bolx I and Bolx II (manufactured by JRT Associates) showed the most promise. Both are approved for patient use because they are non-toxic and non-allergenic. Bolx I, as seen on the right hand side of Figure 21, has a layer of thin, plastic "skin" such that it can be used repeatedly. Conversely, Bolx II as seen on the left hand side of Figure 2-1 is only meant for single patient use. Though the transparent nature of these flexible materials was initially encouraging, they were ultimately abandoned as realistic design alternatives because neither material provided enough downward force when applied to secure the breast or pectoral muscle in place and prevent patient movement. Multiple sources indicate that approximately $50 \%$ of breast cancers occur in the superior lateral (upper outer) quadrant of the breast as indicated in Figure 2-2 [26, 20]. Consequently, it is extremely important to ensure that the breast tissue in this quadrant is held securely in place by the immobilization device. Of equal importance, the breast immobilization device must also generate enough compressive force to secure the pectoral muscle in place so as to guarantee that the breast tissue lining the chest wall will be included in the resulting image. The anatomy of the breast is illustrated in Figure 2-3. 
Providing adequate compressive force motivated us to explore other options above and beyond those discussed thus far.

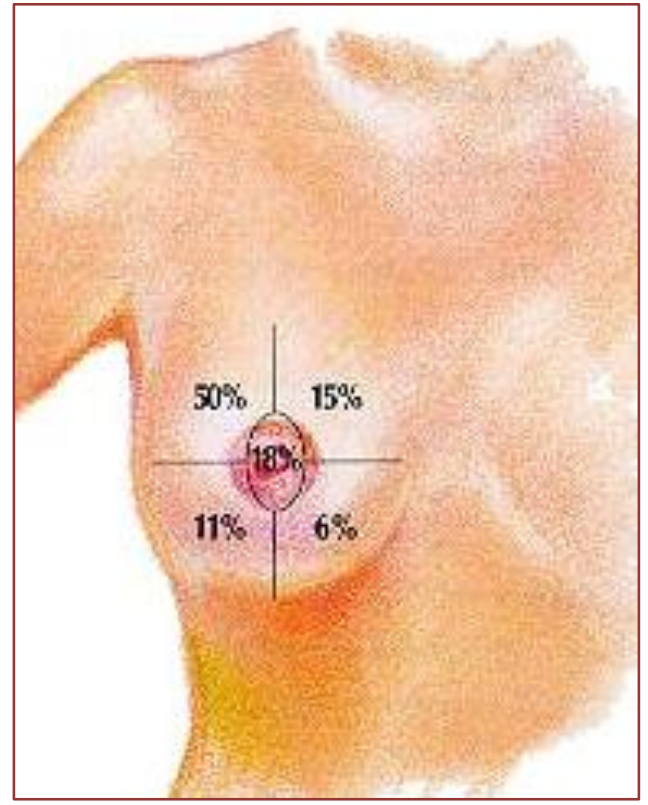

Figure 2-2: Breast Cancer Incidence [26]

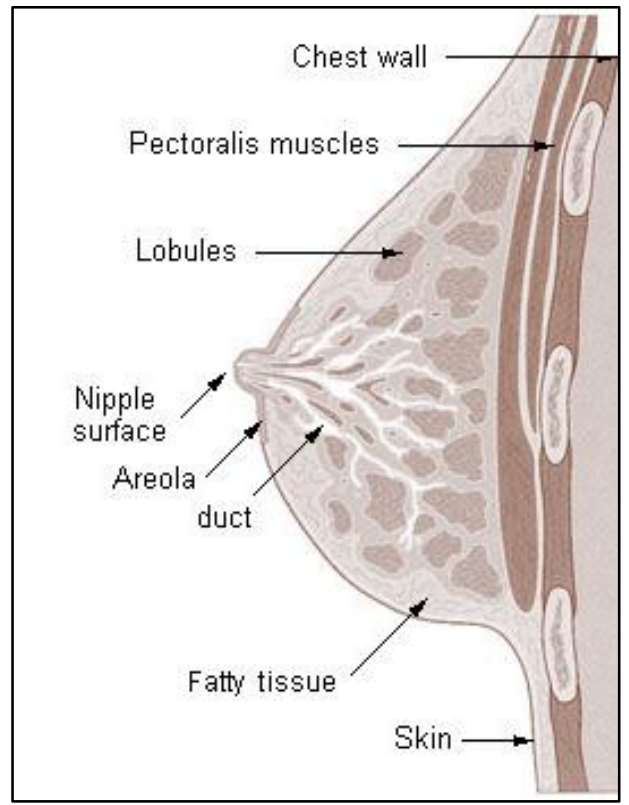

Figure 2-3: Breast Anatomy [21]

\section{A rigid "tongue-and-groove"}

alternative flat paddle design was

temporarily considered as shown in

Figure 2-4. The design consisted of

two-inch thick strips of plastic fastened

together (much like the planks of a

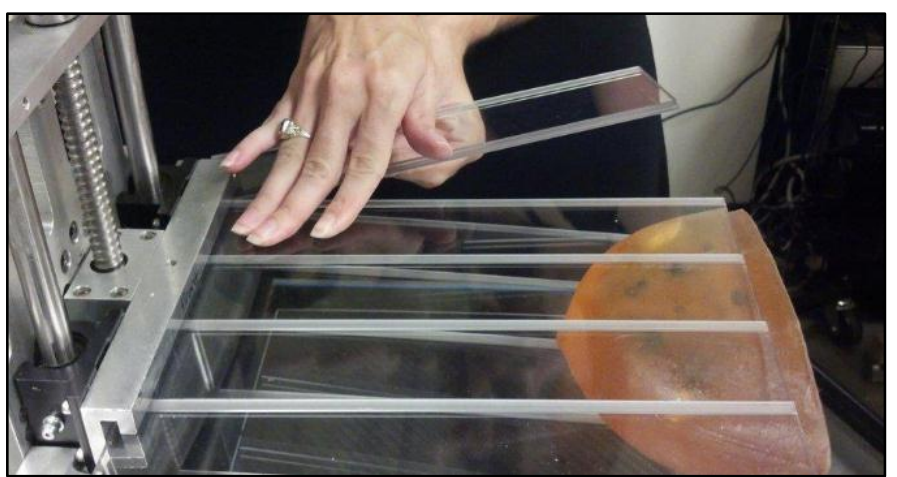

Figure 2-4: Photograph of "tongue-and-groove" alternative design conventional hardwood floor) to form both the compression paddle and the breast support. The goal of this eccentric design was to compress the breast and have the ability to remove the plastic strips that were not above (or below) the breast tissue itself, so that close proximity between the breast and the gamma camera could be obtained at wide-angles. This design alternative was 
eventually abandoned because it could prove unsafe and because the air gaps between the plastic strips were very apparent in the resulting reconstructed image (see Figure 2-5).

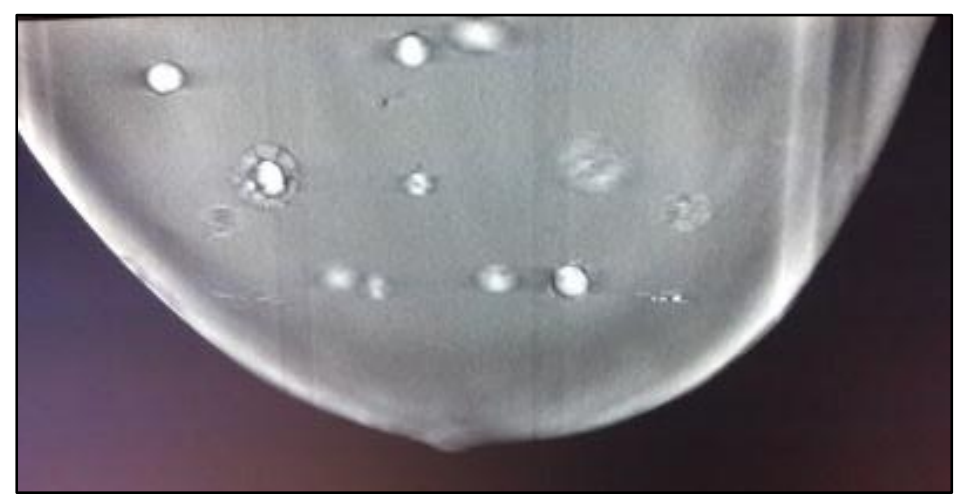

Figure 2-5: "Tongue-and-groove" tomographic image slice

Breast suctioning is oftentimes associated with obstetrics more so than radiology because it is

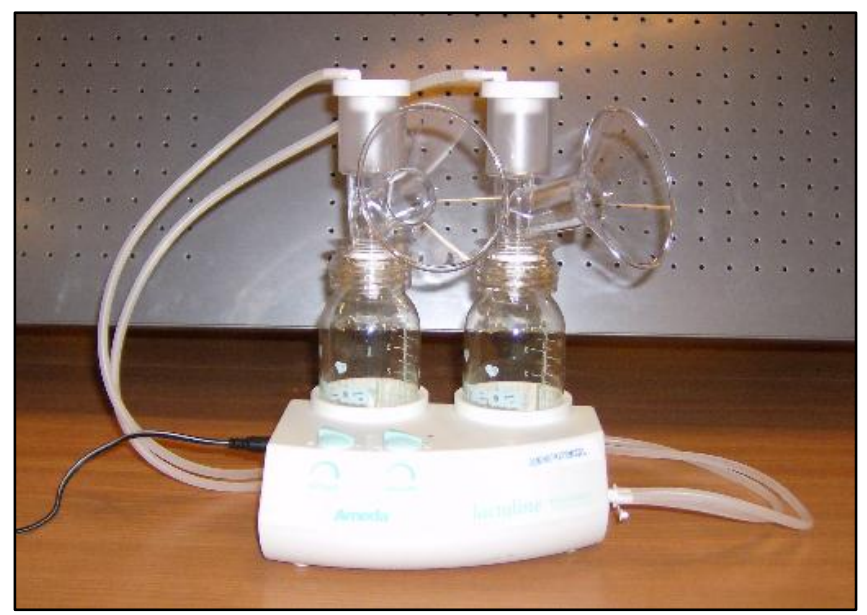

used to pump milk from a woman's breast after she has given birth. A typical breast pump is shown in Figure 2-6. We briefly considered adapting the breast pump to suit our needs; however, two distinct design flaws prevented us from doing so. First, the

Figure 2-6: Breast Pump [1] suction cups themselves are not radiotranslucent, meaning they would show up in the resulting image potentially hindering the radiologist's ability to read the image correctly. Second, the suctioning process does not provide the downward force necessary to include the pectoral muscle (and subsequently the breast tissue at the chest wall) in the acquired image. As a result, this design prospect was discarded in favor of a rigid, curved alternative. 
Though the curved breast immobilization device differs in shape from its flat clinical counterparts, it is manufactured from the same material. Polycarbonate was chosen because of its high strength and ductility. The molecular structure of polycarbonate is fundamentally important to our understanding, characterization, and use of the polymer itself. From a structural standpoint, polycarbonate is comprised of a bisphenol A component and a carbonate group [6]. Figure 2-7 provides good insight into the molecular makeup of polycarbonate. The two

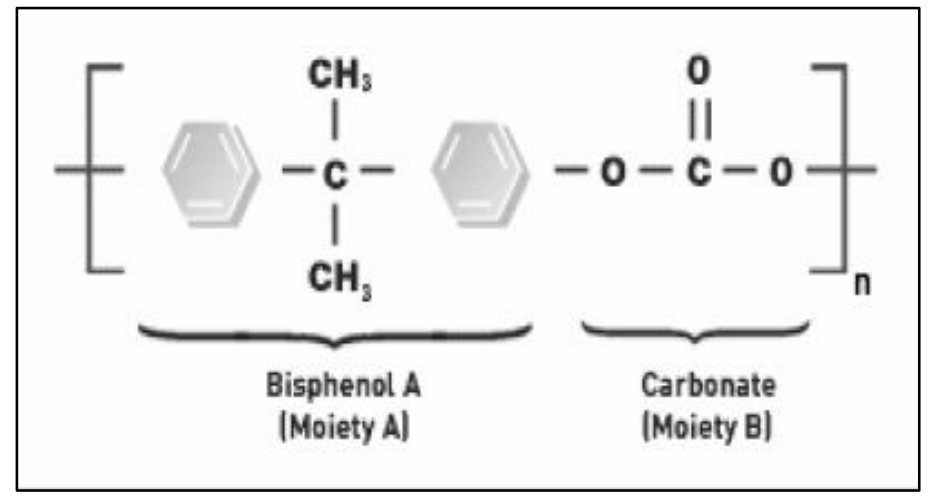

Figure 2-7: Molecular structure of polycarbonate [6] hexagons commonly referred to as

phenyl groups, play a significant role in polycarbonate's ability to deform and flex when subjugated to an applied load. It has been reported that the remarkable ductility of polycarbonate is attributed to a variety of "large-amplitude molecular

motions" including a $180^{\circ}$ phenyl ring flip [24]. The flexibility of polycarbonate is validated by the fact that it has a relatively low Young's modulus, in the vicinity of 2.6 GPa [28]. Young's modulus measures the resistance of a material to elastic (recoverable) deformation under load [7]. A stiff material has a relatively high Young's modulus and changes its shape only slightly when subjected to an elastic load, while a flexible material has a relatively low Young's modulus and changes shape significantly under elastic loads [7].

Stiffness, however, should not be confused with strength. Stiff materials require higher loads to elastically deform, [7] where as strong materials require higher loads to deform permanently [7]. The room temperature compressive strength of polycarbonate is oftentimes reported on industrial websites to approximate $12,000 \mathrm{psi}(\sim 80 \mathrm{MPa})[4,19]$. One particular 
report suggests that the compressive strength of polycarbonate at room temperature is somewhat dependent upon strain rate until high strain rates are realized [2]. Figure 2-8 confirms these findings and validates the compressive strength of polycarbonate as advertised by most suppliers.

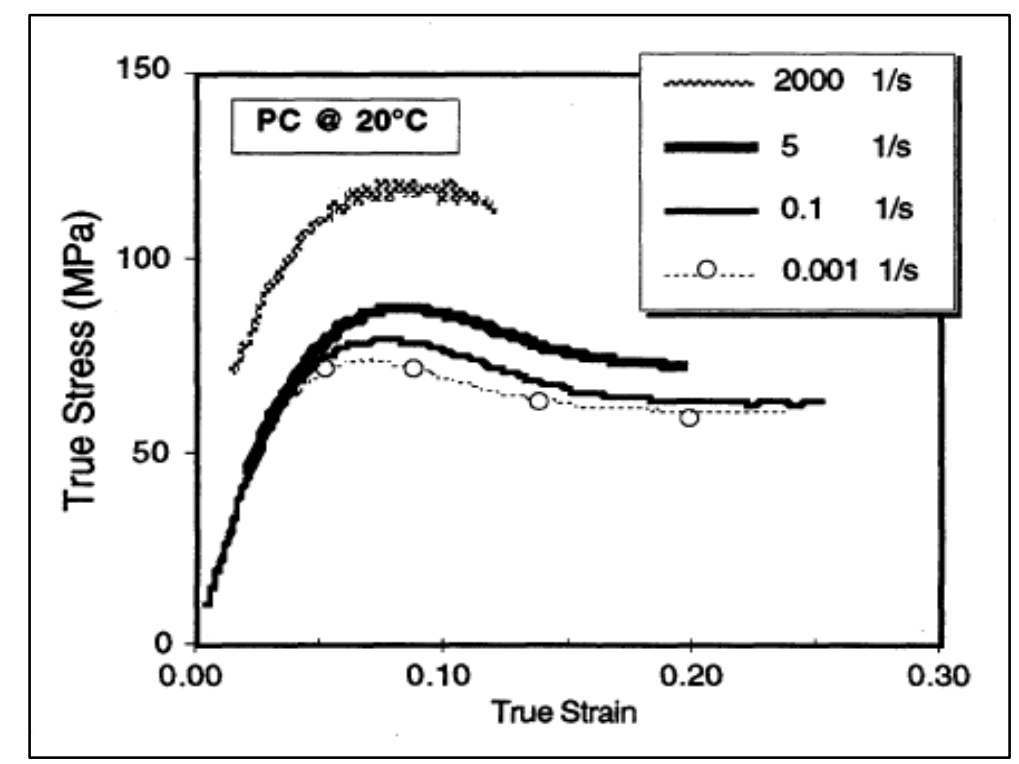

Figure 2-8: Compressive Strength of Polycarbonate [2]

\subsection{Curvature Quantification}

The curvature of the optimized breast immobilization device was empirically derived using an $1100 \mathrm{~mL}$ gelatin breast phantom, a twenty pound ankle weight, and a crudely fabricated curved segment of polycarbonate to quantify the area of an ellipse associated with the phantom breast. To date, our lab has accrued fifty-four human subjects to participate in clinical trial IRBHSB \#8825. The largest breast volume observed thus far is approximately $1100 \mathrm{~mL}$. This volume was calculated as the product of the maximum compressed thickness of the breast observed in the DMT pilot study and an image-based assessment of the maximum projected breast area observed in that study. We chose to use $1100 \mathrm{~mL}$ to quantify the $\mathrm{x}-$ and $\mathrm{y}$ coordinates of an ellipse because this value realistically defines the upper bound breast size 
realized in the pilot DMT study. In addition, we chose to use a twenty pound ankle weight for two main reasons. First, as previously suggested, the average compressive force generated during a clinical exam is twenty pounds. Second, it is important to simulate uniform force distribution to the best of our ability. Figure 2-9 demonstrates the experimental set-up that was used to determine the semi-major and semi-minor axes of an ellipse, ultimately quantifying the $\mathrm{x}$ - and $\mathrm{y}$-coordinates of both the breast support and the compression paddle. An elliptical shape was chosen because it naturally follows the contours of a breast, more so than other geometries.

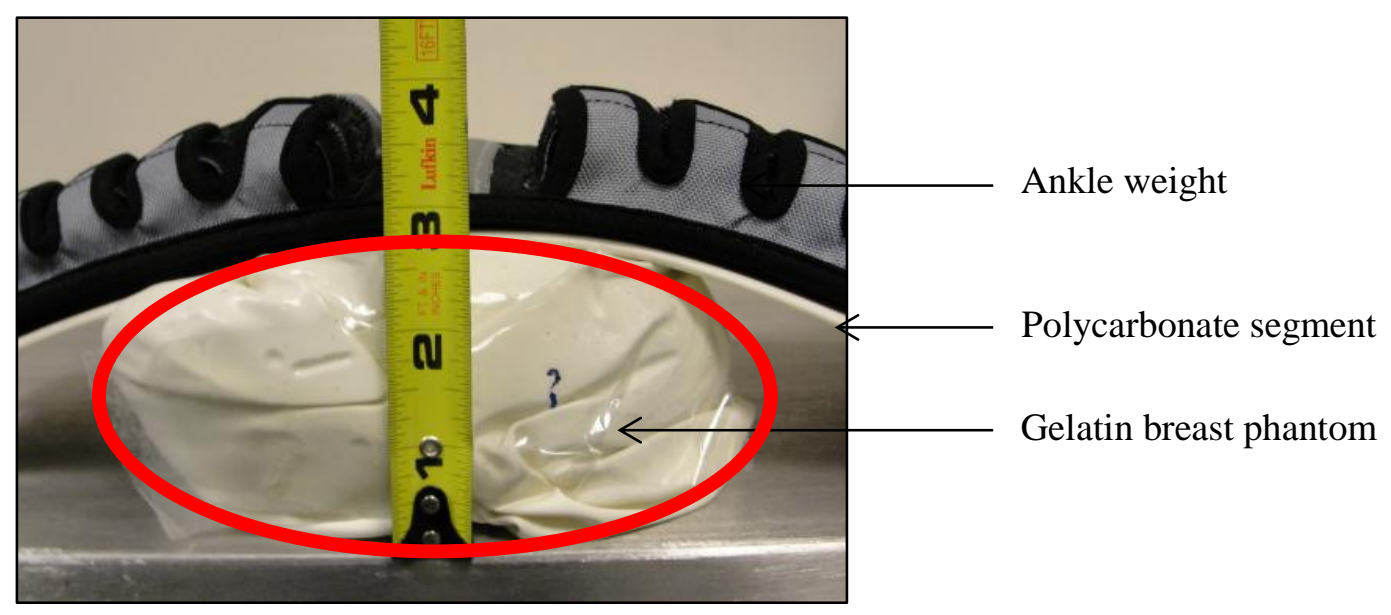

Figure 2-9: Photograph of experimental set-up to determine semi-major and semi-minor axis of an ellipse defining the breast immobilization device

In polar coordinates relative to the center where the angular coordinate $\theta$ is measured from the major axis, the equation of an ellipse described by semi-major axis $a$ and semi-minor axis $b$ can be expressed as,

$$
r(\theta)=\frac{a * b}{\sqrt{(b \cos \theta)^{2}+(a \sin \theta)^{2}}}
$$




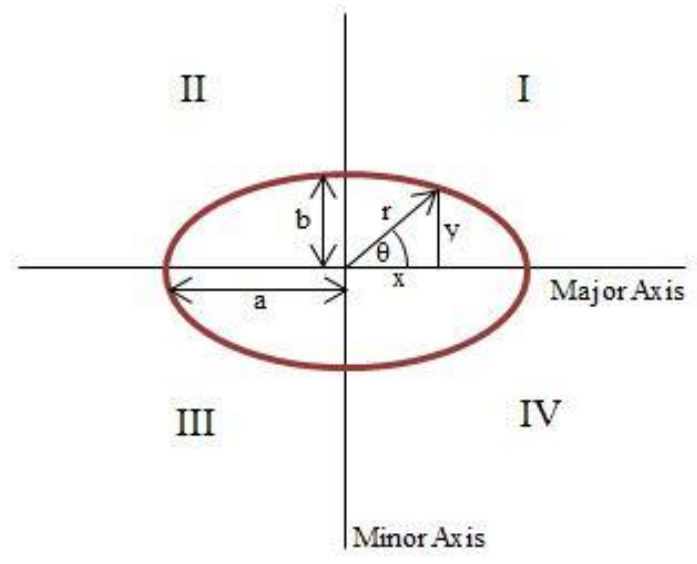

Figure 2-10: Schematic of an ellipse used to derive the $x$ - and $y$-coordinates of the breast immobilization device

From geometry, the $\mathrm{x}$ - and $\mathrm{y}$-coordinates are described as,

$$
\begin{aligned}
& x(\theta)=r \cos \theta \\
& y(\theta)=r \sin \theta
\end{aligned}
$$

Substituting the value of r, equations (2) and (3) can be re-written as,

$$
\begin{aligned}
& x(\theta)=\frac{a * b}{\sqrt{(b \cos \theta)^{2}+(a \sin \theta)^{2}}} \cos \theta \\
& y(\theta)=\frac{a * b}{\sqrt{(b \cos \theta)^{2}+(a \sin \theta)^{2}}} \sin \theta
\end{aligned}
$$

The semi-major axis measured during the experiment is $8.6 \mathrm{~cm}$ (3.39 inches), while the semiminor axis is $4.4 \mathrm{~cm}$ (1.73 inches). Equations (4) and (5) are used in conjunction with parameters $a$ and $b$ to determine the x-and y-coordinates of an ellipse as reported in Appendix A. The coordinate values in quadrants I and II, as defined in Figure 2-10, correspond to points on the curved compression paddle, while those in quadrants III and IV correspond to points on the curved breast support, in effect dividing the ellipse into two equivalent halves down the major 
axis. These semi-elliptical constructs are truncated at either end to accommodate a variety of different breast sizes. Had we not chosen to truncate the optimized breast immobilization device, it would only compress a very limited range of breast sizes and could potentially cause discomfort due to tissue pinching.

\subsection{Truncation Calculations}

In order to truncate the breast immobilization device, the same methods described in Section 2.2 were employed to determine the semi-major and semi-minor axes of an $840 \mathrm{~mL}$ gelatin breast phantom. This value is the average breast volume observed during the pilot DMT study, and as such, it was used to determine the truncation points of the breast immobilization device. The semi-major axis associated with the $840 \mathrm{~mL}$ gelatin breast phantom was measured to be $8.1 \mathrm{~cm}$, while the semi-minor axis was $4.25 \mathrm{~cm}$. For the purposes of this calculation, we required elliptical area conservation, as the area (much like the volume) does not change when compressive force is applied, though the phantom breast will experience reshaping. As such, the semi-major and semi-minor axes of the $840 \mathrm{~mL}$ gelatin breast phantom were used to calculate the area $\left(\mathrm{A}_{1}\right)$ of the associated ellipse. In general, the area of an ellipse can be described as,

$$
A=\pi * a * b
$$

The area, $\mathrm{A}_{1}$, of an ellipse with a semi-major axis equal to $8.1 \mathrm{~cm}$ and a semi-minor axis equal to $4.25 \mathrm{~cm}$ is $108 \mathrm{~cm}^{2}$. The area within the truncated breast immobilization device, $\mathrm{A}_{2}$, must be equal to the area, $\mathrm{A}_{1}$, of the ellipse defined by the $840 \mathrm{~mL}$ gelatin breast phantom (see Figure 2-11). In other words, the truncation points along the major-axis are dictated by the calculated area under the curve, $\mathrm{A}_{2}$, where the red line representing the breast immobilization device (defined by an $1100 \mathrm{~mL}$ gelatin breast phantom) is shifted down to the green line (defined 
by an $840 \mathrm{~mL}$ gelatin breast phantom) in quadrants I and II of Figure 2-11 and shifted up to the green line in quadrants III and IV. The goal of the calculation is to equate $\mathrm{A}_{2}$ to the crosssectional area of the green ellipse $\left(\mathrm{A}_{1}=108 \mathrm{~cm}^{2}\right)$, truncating the breast immobilization device such that the area of the average compressed breast is equal to the area of the optimized device.

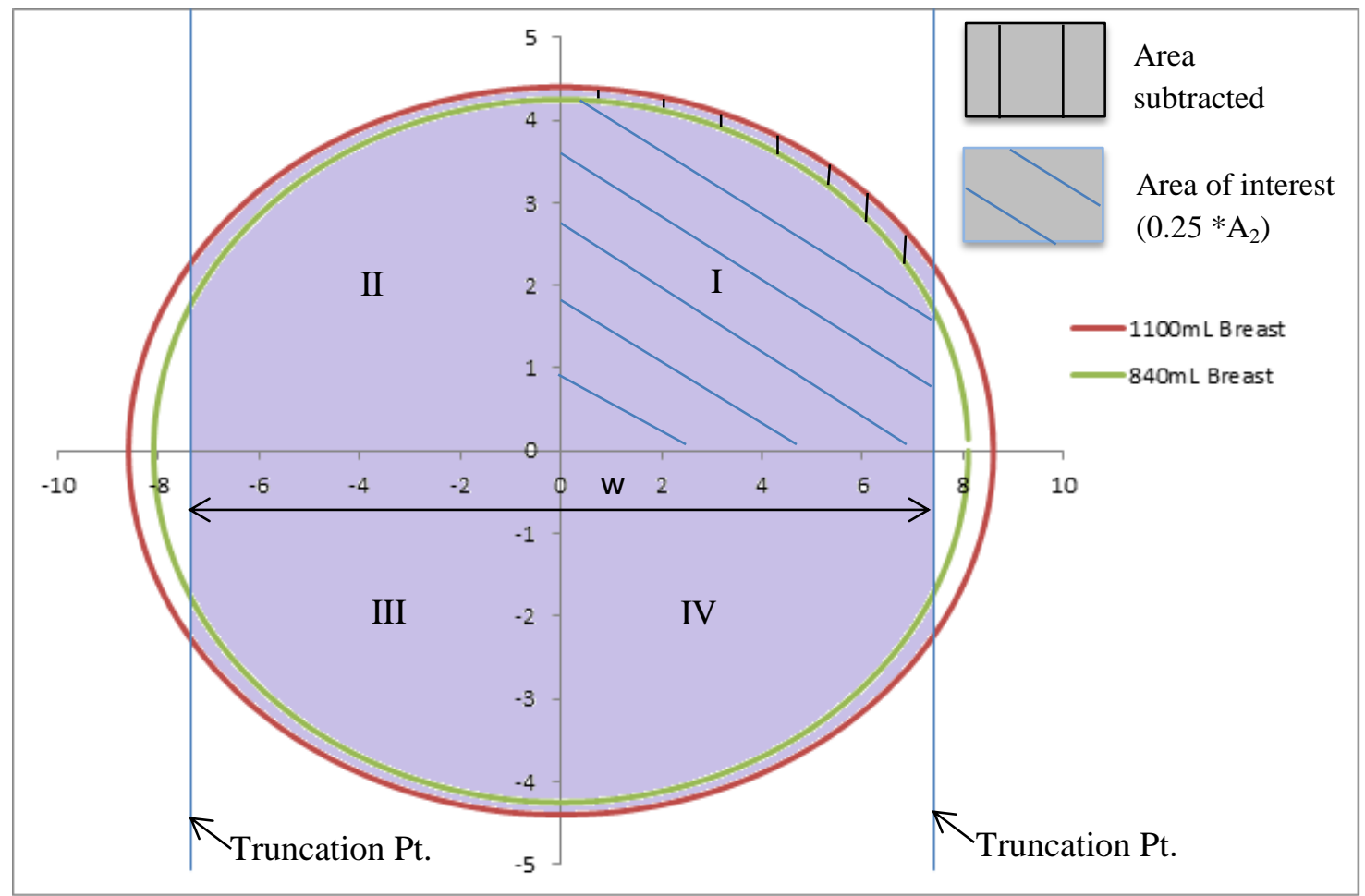

Figure 2-11: Illustration of truncation locations and area within the truncated breast immobilization device

As evident in Figure 2-11, the area under the curve is calculated in quadrant I and subsequently multiplied by four. The equation of an ellipse in Cartesian coordinates is used to calculate the area under the curve and is expressed as,

$$
\left(\frac{x}{a}\right)^{2}+\left(\frac{y}{b}\right)^{2}=1
$$

Isolating the variable $y$ results in the following equation: 


$$
y=\sqrt{1-\frac{x^{2}}{a^{2}}} * b
$$

A delta $(\Delta=0.15 \mathrm{~cm})$, representing the difference between the semi-minor axis associated with the $1100 \mathrm{~mL}$ gelatin breast phantom and the $840 \mathrm{~mL}$ gelatin breast phantom, is incorporated into equation (8) as follows:

$$
y=\left(\sqrt{1-\frac{x^{2}}{a^{2}}} * b\right)-\Delta
$$

Integrating equation (9) from 0 to $w / 2$, where $w$ is the width of the truncated breast immobilization device, we obtain

$$
\int y d x=\int_{0}^{w / 2}\left(\sqrt{1-\frac{x^{2}}{a^{2}}} * b-\Delta\right) d x
$$

Equation (10) can be re-written as

$$
\int y d x=\int_{0}^{w / 2}\left(\sqrt{1-\frac{x^{2}}{a^{2}}} * b\right) d x-\int_{0}^{w / 2} \Delta d x
$$

Simplified further, equation (11) becomes

$$
\int y d x=b \int_{0}^{w / 2}\left(\sqrt{1-\frac{x^{2}}{a^{2}}}\right) d x-\left(\Delta *\left(\frac{w}{2}\right)\right)
$$

From algebra,

$$
\sqrt{1-\frac{x^{2}}{a^{2}}}=\frac{\sqrt{a^{2}-x^{2}}}{a}
$$

Equation (12) can then be expressed as

$$
\int y d x=b \int_{0}^{w / 2}\left(\frac{\sqrt{a^{2}-x^{2}}}{a}\right) d x-\left(\Delta *\left(\frac{w}{2}\right)\right)
$$

Using trigonometric substitution and considering the following triangle: 


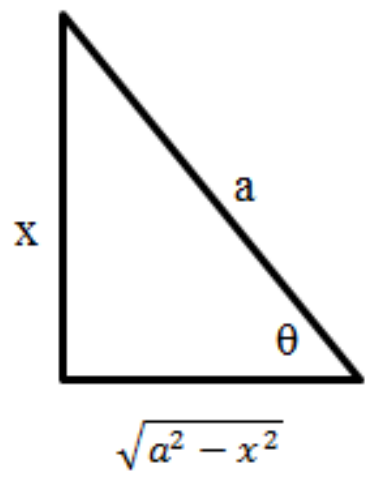

Figure 2-12: Trigonometric Substitution Triangle

Equation (14) can be re-written as

$$
\int y d x=b \int \cos \theta a \cos \theta d \theta-\left(\Delta *\left(\frac{w}{2}\right)\right)
$$

Where $\cos \theta=\frac{\sqrt{a^{2}-x^{2}}}{a}, x=a \sin \theta, d x=a \cos \theta d \theta$, and $\theta=\sin ^{-1}\left(\frac{x}{a}\right)$

Rearranging constants, equation (15) becomes

$$
\int y d x=a b \int \cos ^{2} \theta d \theta-\left(\Delta *\left(\frac{w}{2}\right)\right)
$$

Where $\cos ^{2} \theta=\frac{(1+\cos 2 \theta)}{2}$ such that equation (16) is modified as follows:

$$
\int y d x=a b \int \frac{(1+\cos 2 \theta)}{2} d \theta-\left(\Delta *\left(\frac{w}{2}\right)\right)
$$

Rearranging equation (17), we obtain 


$$
\int y d x=\frac{a b}{2} \int(1+\cos 2 \theta) d \theta-\left(\Delta *\left(\frac{w}{2}\right)\right)
$$

Equation (18) then becomes,

$$
\int y d x=\frac{a b}{2}\left[\theta+\frac{1}{2} \sin 2 \theta\right]-\left(\Delta *\left(\frac{w}{2}\right)\right)
$$

Where $\sin 2 \theta=2 \sin \theta \cos \theta$, such that equation (19) can be re-written as,

$$
\int y d x=\frac{a b}{2}\left[\theta+\frac{1}{2}(2) \sin \theta \cos \theta\right]-\left(\Delta *\left(\frac{w}{2}\right)\right)
$$

Equation (20) can then be expressed in terms of $x$ by,

$$
\int y d x=\frac{a b}{2}\left[\sin ^{-1}\left(\frac{x}{a}\right)+\frac{1}{2}(2)\left(\frac{x}{a}\right)\left(\frac{\sqrt{a^{2}-x^{2}}}{a}\right)\right]_{0}^{w / 2}-\left(\Delta *\left(\frac{w}{2}\right)\right)
$$

$\mathrm{A}_{2}$ is finally expressed as,

$$
A_{2}=4 *\left[\frac{a b}{2} \sin ^{-1}\left(\frac{w}{2 a}\right)+\frac{b w}{4}\left(\sqrt{1-\frac{w^{2}}{4 a^{2}}}\right)-\left(\Delta *\left(\frac{w}{2}\right)\right)\right]
$$

Where $w$ is the truncated width of the breast immobilization device, $\mathrm{a}=8.6 \mathrm{~cm}$ and $\mathrm{b}=4.4 \mathrm{~cm}$.

The points of truncation along the major axis of the ellipse at $+/-w / 2$ were calculated by setting $\mathrm{A}_{2}$ equal to $\mathrm{A}_{1}$. Appendix $\mathrm{B}$ clearly illustrates that $\mathrm{A}_{2}=\mathrm{A}_{1}=108 \mathrm{~cm}^{2}$ when $w / 2=+/-7.5$ $\mathrm{cm}$. As such, the total width of the truncated breast immobilization device $(w)$ is $15 \mathrm{~cm}$. Referring to Appendix A, the theta values ( $\theta$ ) that correspond to $w / 2=+/-7.5 \mathrm{~cm}$ are easily realized. These results suggest that the curved compression paddle should be truncated at $\theta=+16^{\circ}$ and $\theta=+164^{\circ}$ from the positive $\mathrm{x}$-axis, while the curved breast support should be truncated at $\theta=+196^{\circ}$ and $\theta=+344^{\circ}$ from the positive $\mathrm{x}$-axis. 


\subsection{Detailed Device Description and Fabrication}

A prototype breast immobilization device was fabricated by Virginia Industrial Plastics Incorporated (Elkton, Virginia) and is shown in Figure 2-13. As previously described, the

prototype is

comprised of a

curved compression

paddle and a curved

breast support, both

of which are

vacuum formed

from 0.125 inch

thick polycarbonate.

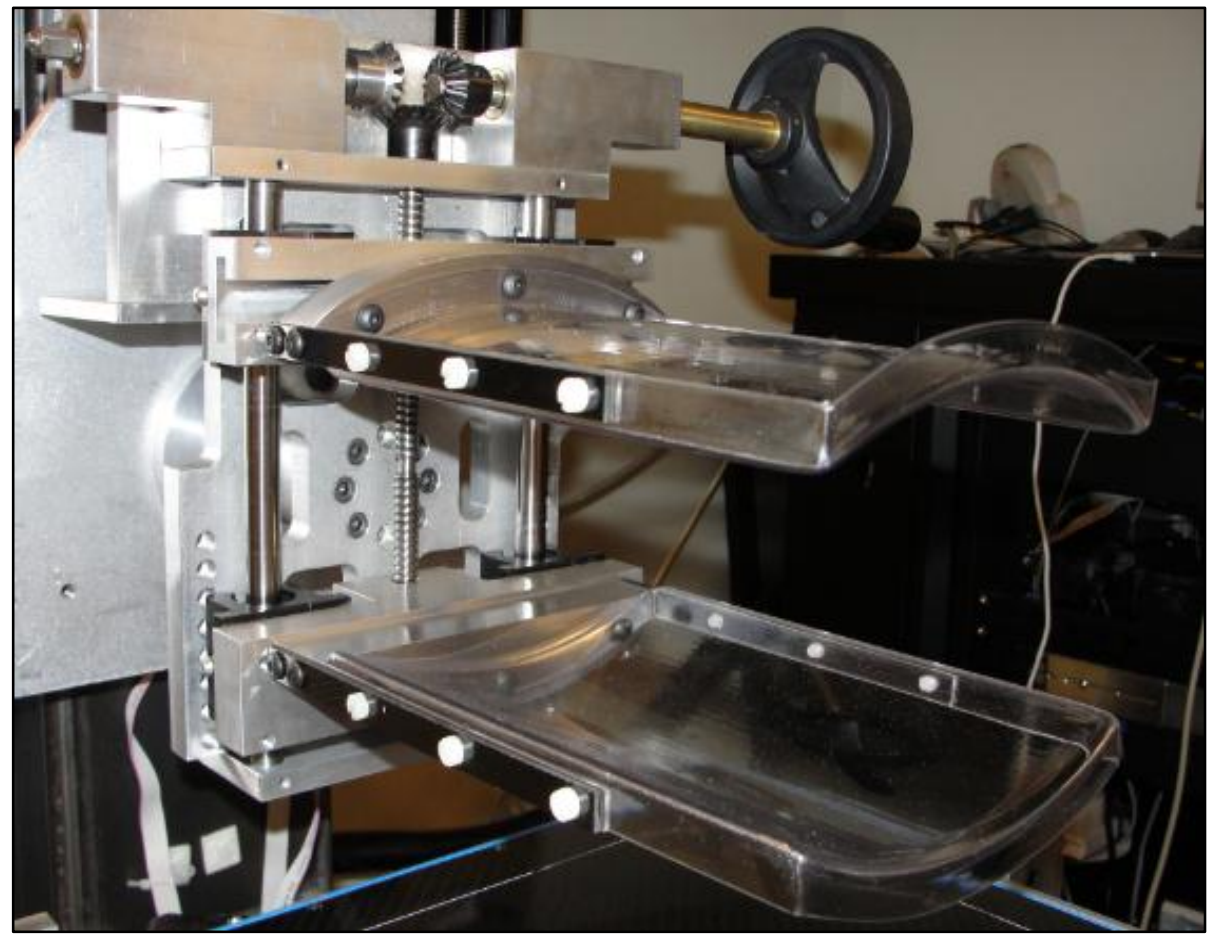

The vacuum

Figure 2-13: Photograph of Optimized Breast Immobilization Device

forming process

involves the following steps. First, a mold is made for the component part [31]. Then, the mold is placed into a machine and the polycarbonate sheet clamped into the frame [31]. Heat is then applied to the polycarbonate until it becomes pliable [31]. Finally, the softened polycarbonate sheet is lowered onto the mold, at which time suction is applied [31]. Upon completion of the component part, it is dismounted from the machine and precision trimmed [31].

The overall dimensions of the curved compression paddle and the curved breast support are 7.75 inches long x 5.91 inches wide x 1.26 inches high. Though the width (x-dimension) of the device was dictated by the location of the truncation points and the height was dictated by 
both the semi-minor axis of the ellipse and the location of the truncation points, the length (ydimension) of the device was chosen in keeping with the length of the standard flat paddle used to compress the breast during the pilot DMT study. To ensure that all parts of the breast anatomy are held securely in place during the scan, rigidity was added into the design by including a 0.375 inch high lip along the perimeter edge of both component parts. A detailed drawing of the breast immobilization device is included in Appendix C.

The curved compression paddle and curved breast support are mounted to the gantry by identical support mechanisms facing in opposite directions and centered on the axis of rotation (AOR) of the DMT scanner. The compression paddle is oriented such that it is concave downwards (in the negative z-direction), while the breast support is oriented such that it is concave upwards (in the positive z-direction). The support mechanisms are fabricated from aluminum and rigid carbon fiber components. The overall dimensions of the support pieces are dictated by the dimensions of the breast immobilization device itself and are disclosed in the support system drawings included in Appendix C. The curvature of the aluminum part is determined by the semi-major and semi-minor axes of the ellipse that defines the shape of the breast immobilization device. The truncation points for the aluminum part are the same as those outlined for the breast immobilization device.

The support mechanism that integrates the compression paddle into the gantry is mounted to a right handed ball lead screw, while the support mechanism that integrates the breast support into the gantry is mounted to a left handed ball lead screw whose pitch is the same as that of the right handed lead screw. The right handed ball lead screw is coaxial with and rigidly attached to the left handed ball lead screw. The lead screw axes intersect the AOR and are parallel to the zaxis. A single mechanical crank system translates the paddle in the negative z-direction while 
simultaneously translating the support in the positive z-direction. Thus, the paddle and the support move in opposite directions at equal speeds and by equal distances towards the AOR. The immobilized breast is centered on the AOR when fully immobilized. 
CHAPTER 3

\section{GELATIN BREAST PHANTOM AND GAMMA CAMERA TRAJECTORY STUDIES}

Gelatin breast phantom and gamma camera trajectory studies were performed to evaluate the effectiveness of the prototype breast immobilization device by comparing the reconstructed MBIT images associated with a camera trajectory dictated by the optimized breast immobilization device with those of standard, flat paddle compression. At the time of the experiment, the gamma camera used in the pilot DMT study was not available, because it was being repaired by our collaborators at West Virginia University. Therefore, a similar camera was employed for the purposes of this study. Figure 3-1 illustrates the experimental set-up that was used to obtain projection data for each of the aforementioned trajectories. Of particular importance, the immobilization devices themselves were not used in the set-up because Virginia 
Plastics Inc. was concurrently fabricating the optimized breast immobilization device. However, a thin-walled, low-attenuation acrylic box was used to simulate flat paddle compression.

\subsection{Study Design}

\subsubsection{Experimental Set-Up}

Note: significant portions of Section 3.1.1 were taken with permission from reference [13]

The gamma camera that was selected for use and built at Jefferson Labs (Newport News, VA), consists of a 3 x 4 array of Hamamatsu H8500 PSPMTs optically coupled to a NaI (TI) crystal array with a crystal pitch of $1.4 \mathrm{~mm}$ and a crystal thickness of $6 \mathrm{~mm}$. The camera is also equipped with a lead parallel hole collimator that has an associated efficiency of $1.10 \times 10^{-4}$, while the acquisition is controlled by software written in K-max (Sparrow Corporation, Port Orange, FL). The camera was positioned in front of a computer-controlled rotation stage mounted on a linear translation stage, which allowed us to vary both the viewing angle and the AOR-to-camera distance to simulate the motion of the gamma camera in the DMT system. COSMOS, a motor controller software program written by Velmex, was used to rotate as well as translate the gelatin breast phantom to specific locations so as to acquire the projection data needed to reconstruct the MBIT images. An $840 \mathrm{~mL}$ radioactive gelatin breast phantom was used in this experiment as illustrated in Figure 3-1. The phantom contained two plastic, thin-walled spherical lesions with an inside diameter of $15 \mathrm{~mm}$.

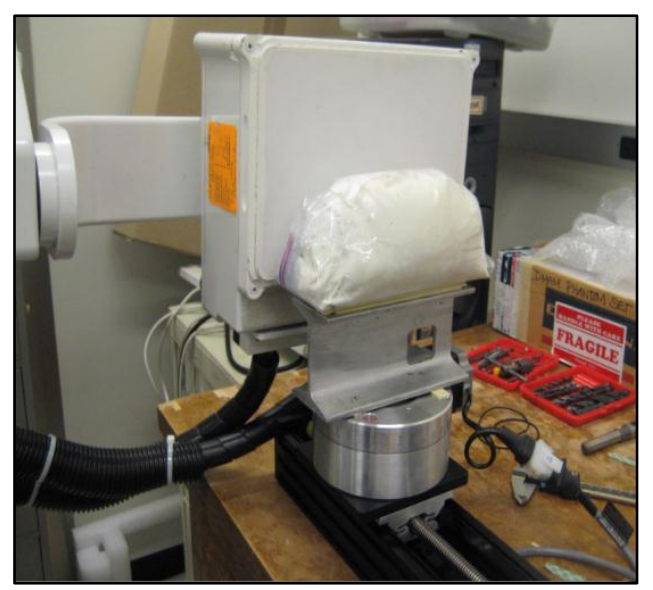

Figure 3-1: Photograph of Experimental Set-up 


\subsubsection{Activity and Acquisition Time Calculations}

During a two minute clinical scan, we typically see an activity concentration of 0.33

$\mu \mathrm{Ci} / \mathrm{mL}$ within the normal breast tissue. In the interest of efficiency, the desired background

activity concentration was doubled $(0.67 \mu \mathrm{Ci} / \mathrm{mL})$ for these experiments to reduce the

acquisition time associated with each projection view. The lesion-to-background activity

concentration ratio that was chosen for these experiments was 10:1. The total activity at the time

of the experiment was determined by multiplying the desired concentrations by the appropriate

\begin{tabular}{|c|c|c|c|c|c|c|c|c|c|c|c|c|c|}
\hline & \multicolumn{13}{|c|}{ Data as Input } \\
\hline & \multicolumn{13}{|c|}{ Calculated Data } \\
\hline & \multicolumn{13}{|c|}{ Required Quantities for experiment } \\
\hline & & & & & & & & & & & & & \\
\hline \multicolumn{2}{|c|}{$\begin{array}{c}\text { Breast } \\
\text { tissue vol. }\end{array}$} & \multicolumn{2}{|c|}{ Lesion vol. } & \multicolumn{3}{|c|}{ Desired Concentration } & \multicolumn{2}{|c|}{$\begin{array}{l}\text { Total Act. @ } \\
\text { time of exp. }\end{array}$} & \multicolumn{2}{|c|}{$\begin{array}{c}\text { Hours } \\
\text { Before } \\
\text { exp. }\end{array}$} & \multicolumn{2}{|c|}{$\begin{array}{l}\text { Total Act. to put } \\
\text { in }\end{array}$} & $\begin{array}{c}\text { Packets } \\
\text { of jello } \\
\text { Reqd. }\end{array}$ \\
\hline [cups] & {$[\mathrm{mL}]$} & [cups] & {$[\mathrm{mL}]$} & $\begin{array}{c}B k g \\
{[\mu C i / m L]}\end{array}$ & \begin{tabular}{|c|} 
Ratio \\
(Lesion \\
:Bkg)
\end{tabular} & $\begin{array}{c}\text { Lesion[ } \mu C i \\
/ m L]\end{array}$ & $\begin{array}{c}B k g \\
{[\mu C i]}\end{array}$ & $\begin{array}{c}\text { Lesion } \\
{[\mu \mathrm{Ci}]}\end{array}$ & hrs & $\min$ & $\begin{array}{c}B k g \\
{[\mu C i]}\end{array}$ & $\begin{array}{c}\text { Lesion } \\
{[\mu \mathrm{Ci}]}\end{array}$ & $B \mathrm{~kg}[\#]$ \\
\hline 3.5 & 840 & 0.5 & 120 & 0.67 & 10 & 6.7 & 563 & 804 & 5 & 0 & 1000.9 & 1429.8 & 5.25 \\
\hline
\end{tabular}

Table 3-1: Spreadsheet calculating background and lesion activity

volume, as illustrated in Table 3-1. Since the activity is injected into gelatin that is initially in liquid form, the experiments were not conducted until a few hours later, after the gelatin had set.

${ }^{99 \mathrm{~m}}$ Tc-pertecnitate, the radioactive isotope used in these studies, has a half-life of approximately

six hours. As such, we had to account for decay when calculating the initial amount of

radioactivity required for both the lesions and the phantom itself by estimating the requisite gelatin set time based on our intuition and experience. The Beer-Lambert Law, which can be expressed as,

$$
A(t)=A_{0} e^{-\lambda t}
$$


was used to compute the total initial activity needed by first rearranging the variables to obtain equation (24).

$$
\lambda=-\frac{L N\left(\frac{A_{\text {thalf }}}{A_{o}}\right)}{t_{\text {half }}}=-\frac{L N\left(\frac{1}{2}\right)}{t_{\text {half }}}=\frac{L N(2)}{t_{\text {half }}}=\frac{L N(2)}{6.02}
$$

Equation (24) is the half-life of ${ }^{99 \mathrm{~m}}$ Tc-pertecnitate and can be substituted back into equation (23) to subsequently solve for $A_{0}$, where $t=5$ hours and $A(t)$ is the total activity at the time of the experiment. The Beer-Lambert Law then becomes,

$$
A_{o}=A(t) e^{\frac{L N(2)}{6.02} t}
$$

Finally, the total number of gelatin packets needed to create the breast phantom was computed by multiplying 1.5 by the breast tissue volume (in cups). The lesions were filled with radioactive water rather than gelatin to facilitate easy cleanup at the end of the experiments.

After the gelatin phantom had set, the image acquisition time had to be calculated before beginning the experiments. Again, the Beer-Lambert Law was employed in Table 3-2 to

\begin{tabular}{|c|c|c|c|c|c|c|c|c|c|c|}
\hline \multicolumn{11}{|l|}{ Input: } \\
\hline & \multicolumn{9}{|c|}{ * Format of input: TIME(hours, minutes,sec) with hours in military time } & \\
\hline & & & & & & & & & & \\
\hline \multirow[t]{2}{*}{ Output: } & & \multicolumn{9}{|c|}{ Value to put into Kmax program for acquisition time for single projection } \\
\hline & & & & & & & Concen & ntration [ & $\mu \mathrm{Ci} / \mathrm{mL}]$ & \\
\hline $\begin{array}{c}\text { Phantom } \\
\text { Vol. }\end{array}$ & $\begin{array}{c}\text { Syringe } \\
\text { Act B4 } \\
\text { added } \\
\text { to jello } \\
\text { [ } \mu \mathrm{Ci}]\end{array}$ & $\begin{array}{c}\text { Syringe } \\
\text { Act after } \\
\text { added to } \\
\text { jello } \\
{[\mu \mathrm{Ci}]}\end{array}$ & $\begin{array}{c}\text { Total } \\
\text { Added } \\
\text { Activity } \\
{[\mu C i]}\end{array}$ & $\begin{array}{c}\text { Time } \\
\text { Act } \\
\text { Added* }\end{array}$ & $\begin{array}{c}\text { Time } \\
\text { Exper. } \\
\text { Started* }\end{array}$ & $\begin{array}{c}\text { Time } \\
\text { Elapsed }\end{array}$ & $\begin{array}{c}\text { When } \\
\text { added } \\
\text { to jello }\end{array}$ & $\begin{array}{c}\text { Exper. } \\
\text { Start }\end{array}$ & $\begin{array}{l}\text { Desired } \\
\text { Concen. } \\
{[\mu \mathrm{Ci} / \mathrm{ml}]}\end{array}$ & $\begin{array}{c}\text { Required } \\
\text { Time [s] }\end{array}$ \\
\hline 840 & 1001 & 0 & 1001 & 0.392 & 0.583 & 4.600 & 1.1917 & 0.7017 & 0.33 & 56.43692 \\
\hline
\end{tabular}
determine the concentration of activity in the gelatin phantom at the start of the experiment.

Table 3-2: Spreadsheet calculating image acquisition time 
The two parameters that were needed to compute $\mathrm{A}\left(\mathrm{t}_{\mathrm{exp}}\right)$ included the concentration of activity in the gelatin phantom at the time that it was added and the time that had elapsed between the addition of activity and the commencement of the experiments themselves. Once $\mathrm{A}\left(\mathrm{t}_{\mathrm{exp}}\right)$ had been determined, the desired concentration dictated by clinical observation, $0.33 \mu \mathrm{Ci} / \mathrm{mL}$, and clinical scan time, 120 seconds per image acquired, were used to calculate the acquisition time for the very first image obtained during the experiment. In order to account for decay over the time course of the entire experiment, this acquisition time was entered into the K-max GUI, as illustrated in Figure 3-2, to determine the associated number of counts, or total events, in the first image. This number was subsequently held constant for all of the remaining acquisitions.

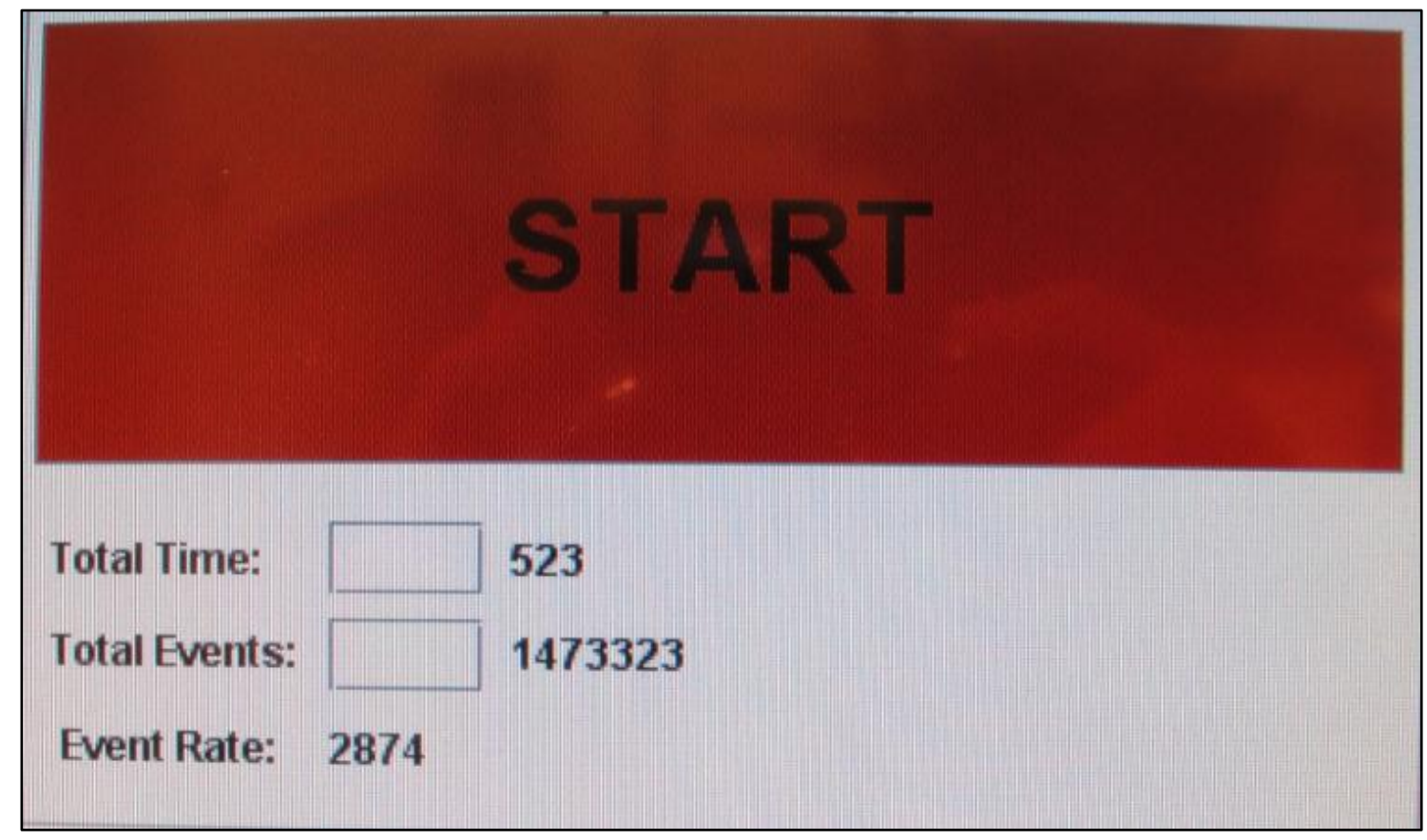

Figure 3-2: K-max GUI 


\subsubsection{Trajectory Calculations}

As previously mentioned, two gamma camera trajectories were calculated to determine the relative position of the camera with respect to the gelatin breast phantom. The first trajectory was dictated by the flat compression paddle and breast support that were used in the pilot DMT study, while the second trajectory was dictated by the optimized breast immobilization device. Two assumptions were made with respect to these trajectories. We assumed that the center normal of the detector always intersected the AOR, and that the projection of the detector surface is tangent to the immobilization device at every projection angle. These trajectories were evaluated between $-67.5^{\circ}$ and $67.5^{\circ}$ at angular increments of $5.625^{\circ}$. Twenty-five projection views were acquired during the scan, including a zero degree view, though only nine of these projection views were used to reconstruct the 3-D image. A series of Monte Carlo simulations were performed by Zongyi Gong, a friend and fellow colleague, to determine the optimal acquisition angle range $\left(+/-67.5^{\circ}\right)$ and number of projection views (9), as he perfected the MBIT reconstruction algorithm that he wrote and developed. Considering Figure 3-3, the "flat paddle" trajectory was calculated as follows:

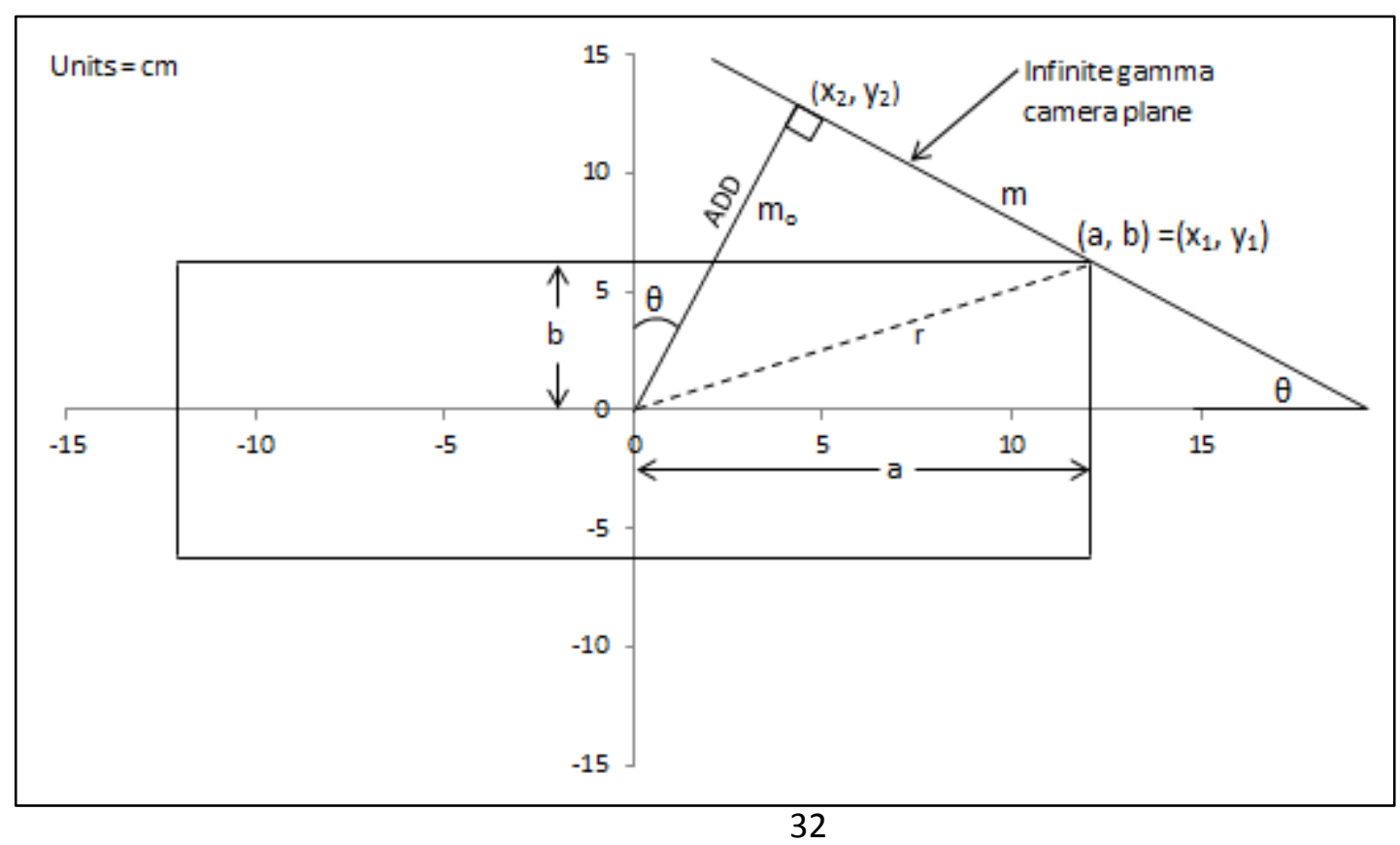

Figure 3-3: "Flat Paddle" Trajectory Schematic 
By definition, the slope of the line defining the gamma camera plane is,

$$
m=\tan (\theta)
$$

Furthermore, because the line defining the AOR to detector distance (ADD) is perpendicular to the line defining the gamma camera plane, its slope can be defined as the negative reciprocal of $m$.

$$
m_{o}=-\frac{1}{m}=-\frac{1}{\tan (\theta)}
$$

The slope-intercept form of a line can be used to describe ADD because both the slope and yintercept of the line are known.

$$
y=m_{o} x=-\frac{1}{\tan \theta} x
$$

The point-slope form of a line can be used to describe the camera detector plane because both the slope and the point $(\mathrm{a}, \mathrm{b})$ are known. Point $a$ is simply half of the width of the flat compression paddle, whereas point $b$ is half of the average compressed breast thickness observed during the pilot DMT study plus half the thickness of the breast support and compression paddle combined.

$$
y-b=m(x-a)
$$

Equation (29) can be expressed as,

$$
y=m x-m a+b
$$

The location $\left(\mathrm{x}_{2}, \mathrm{y}_{2}\right)$ on the camera can be found by setting equation (28) equal to (30).

$$
m_{0} x_{2}=m x_{2}-m a+b
$$


Equation (31) can be rearranged to solve for $x_{2}$ as follows:

$$
x_{2}=\frac{-m a+b}{m_{o}-m}=\left(\frac{-((\tan \theta * a)+b)}{-\frac{1}{\tan \theta}-\tan \theta}\right)
$$

Substituting equation (32) into equation (28) yields

$$
y_{2}=m_{o}\left(\frac{-m a+b}{m_{o}-m}\right)=-\frac{1}{\tan \theta}\left(\frac{-((\tan \theta * a)+b)}{-\frac{1}{\tan \theta}-\tan \theta}\right)
$$

Given $\theta$, the gamma camera trajectory values associated with "flat paddle" compression $\left(\mathrm{x}_{2}, \mathrm{y}_{2}\right)$ are presented in Appendix D. Similarly, the trajectory associated with the optimized breast immobilization device was computed by considering Figure 3-4.

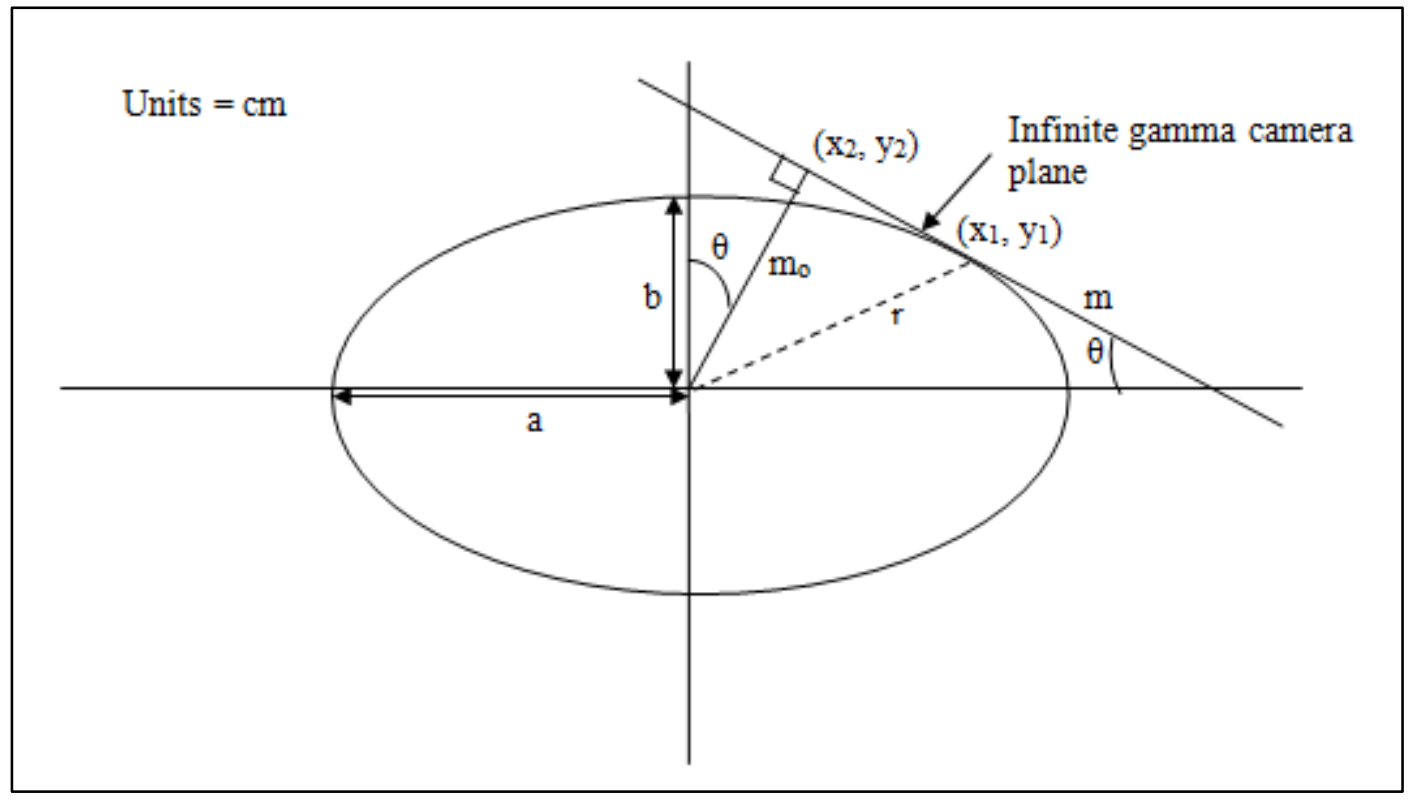

Figure 3-4: Optimized Breast Immobilization Device Trajectory Schematic

Again by definition, the slope of the line defining the camera detector plane is

$$
m=\tan (\theta)
$$


The slope of the line defining the AOR to detector distance (ADD) is once more,

$$
m_{o}=-\frac{1}{m}=-\frac{1}{\tan (\theta)}
$$

In order to calculate $x_{1}$, the equation of an ellipse in Cartesian coordinates is manipulated to yield equation (36) where $a$ is half of the width of the $1100 \mathrm{~mL}$ gelatin breast phantom plus the height of the lip of the optimized breast immobilization device and $b$ is half of the thickness of the 1100 mL gelatin breast phantom plus the height of the lip of the optimized breast immobilization device.

$$
y=\sqrt{1-\frac{x^{2}}{a^{2}}} * b
$$

Taking the derivative of equation (36) with respect to the variable $x$, we obtain the following:

$$
\frac{d y}{d x}=b\left(\frac{d}{d x}\left(\sqrt{1-\frac{x^{2}}{a^{2}}}\right)\right)
$$

Using the chain rule where $\frac{d \sqrt{u}}{d u} * \frac{d u}{d x}, u=1-\frac{x^{2}}{a^{2}}$ and $\frac{d \sqrt{u}}{d u}=\frac{1}{2} * u^{-\frac{1}{2}}=\frac{1}{2 \sqrt{u}}$, equation (37) can be expressed as,

$$
\frac{d y}{d x}=\frac{b\left(\frac{d}{d x}\left(1-\frac{x^{2}}{a^{2}}\right)\right)}{2 \sqrt{1-\frac{x^{2}}{a^{2}}}}
$$

Therefore, the derivative can be re-written as,

$$
\frac{d y}{d x}=\frac{-b(2 x)}{2 a^{2} \sqrt{1-\frac{x^{2}}{a^{2}}}}
$$


Finally, equation (39) becomes

$$
\frac{d y}{d x}=\frac{-b x}{a^{2} \sqrt{1-\frac{x^{2}}{a^{2}}}}
$$

Equation (39) is the slope of the line defining the gamma camera plane and can be re-written as follows:

$$
\frac{d y}{d x}=m=\tan (\theta)=\frac{-b x}{a^{2} \sqrt{1-\frac{x^{2}}{a^{2}}}}=\frac{-b x}{a\left[a^{2}\left(1-\frac{x^{2}}{a^{2}}\right)\right]^{\frac{1}{2}}}
$$

By squaring equation (40), we obtain

$$
m^{2}=\frac{b^{2} x^{2}}{a^{2}\left(a^{2}-x^{2}\right)}=\frac{b^{2} x^{2}}{a^{4}-a^{2} x^{2}}
$$

By rearranging the equation and isolating the variable $x$, equation (41) becomes

$$
x^{2}=\frac{m^{2} a^{4}}{m^{2} a^{2}+b^{2}}
$$

As a result, we obtain the following expression for $\mathrm{x}_{1}$.

$$
x_{1}= \pm \sqrt{\frac{m^{2} a^{4}}{m^{2} a^{2}+b^{2}}}
$$

Equation (43) can then be substituted into equation (36) to solve for $\mathrm{y}_{1}$. Subsequently, the parameters $\mathrm{x}_{2}$ and $\mathrm{y}_{2}$ can be computed as a function of $\mathrm{x}_{1}$ and $\mathrm{y}_{1}$, by setting the equation of a line describing $\operatorname{ADD}\left(\mathrm{y}_{2}=\mathrm{m}_{0} \mathrm{x}_{2}\right)$ equal to the equation of a line describing the gamma camera plane $\left(\mathrm{y}-\mathrm{y}_{1}\right)=\mathrm{m}\left(\mathrm{x}_{2}-\mathrm{x}_{1}\right)$

$$
m_{0} x_{2}=m x_{2}-m x_{1}+y_{1}
$$


Solving for $x_{2}$, we obtain

$$
x_{2}=\frac{-m x_{1}+y_{1}}{m_{o}-m}
$$

Equation (45) can then be substituted into $\mathrm{y}_{2}=\mathrm{m}_{0} \mathrm{x}_{2}$ to calculate $\mathrm{y}_{2}$. The gamma camera trajectory values associated with the optimized breast immobilization device $\left(\mathrm{x}_{2}, \mathrm{y}_{2}\right)$ are also presented in Appendix D.

\subsection{Reconstruction Algorithm Description}

Note: Significant portions of Section 3.2 were taken from a personal interview with Zongyi Gong (2012, June 19).

The reconstruction algorithm that was used to create the 3-D image from the projection data acquired during the experiment was written in Matlab by Zongyi Gong. The Maximum Likelihood Expectation Maximization (MLEM) algorithm is an iterative, statistics based approach to image reconstruction. This algorithm differs significantly from the popular analytical method called filter back projection. Filter back projection will not prevent the transfer of noise from the projection images into the 3-D volume. Because we deal with fairly noisy projection images, the MLEM method is preferred. Generally speaking, the algorithm iteratively finds the 3-D reconstructed volume that has the highest probability of returning the experimentally obtained projection images.

The MLEM approach involves three main steps. First, the algorithm calculates important geometries, such as phantom location, detector location, and AOR-to-detector distance. Second, it chooses an initial, uniform 3-D volume that will change with each successive iteration. Finally, it simulates forward projection by virtually rotating the detector to produce computergenerated projection images from the 3-D volume. These projection images may differ from those acquired during the experiment. These differences are incorporated into a "changing 
factor," which is then multiplied by the current 3-D volume to create a new 3-D volume. This iterative, forward projection approach is repeated until the "changing factor" is zero, in effect providing a true representation of the 3-D volume. Furthermore, the MLEM algorithm models the emitted photon as a Gaussian function instead of a delta function in the forward projection to provide attenuation correction and resolution recovery in the resulting 3-D image.

\subsection{Results}

A comparison study was conducted to evaluate the effectiveness of the optimized breast immobilization device once the MBIT images had been reconstructed using the MLEM algorithm. The image quality figures of merit included lesion contrast and signal-to-noise ratio (SNR). Lesion intensity and background intensity were measured in single slices of the 3-D reconstructed volume where the lesion intensity appeared brightest. Lesion intensity was defined as the average pixel value in a $4 \times 4$ pixel ROI centered on the lesion, while background intensity was defined as the average background pixel value in a $30 \times 20$ pixel ROI near the lesion of interest. Figures 3-5 and 3-6 respectively illustrate a lesion ROI and background ROI in a single slice image for the gamma camera trajectory defined by the optimized breast immobilization device. Similar images were acquired and analyzed for the gamma camera trajectory defined by the standard, flat compression paddle. A total of five trials were conducted for each trajectory. 


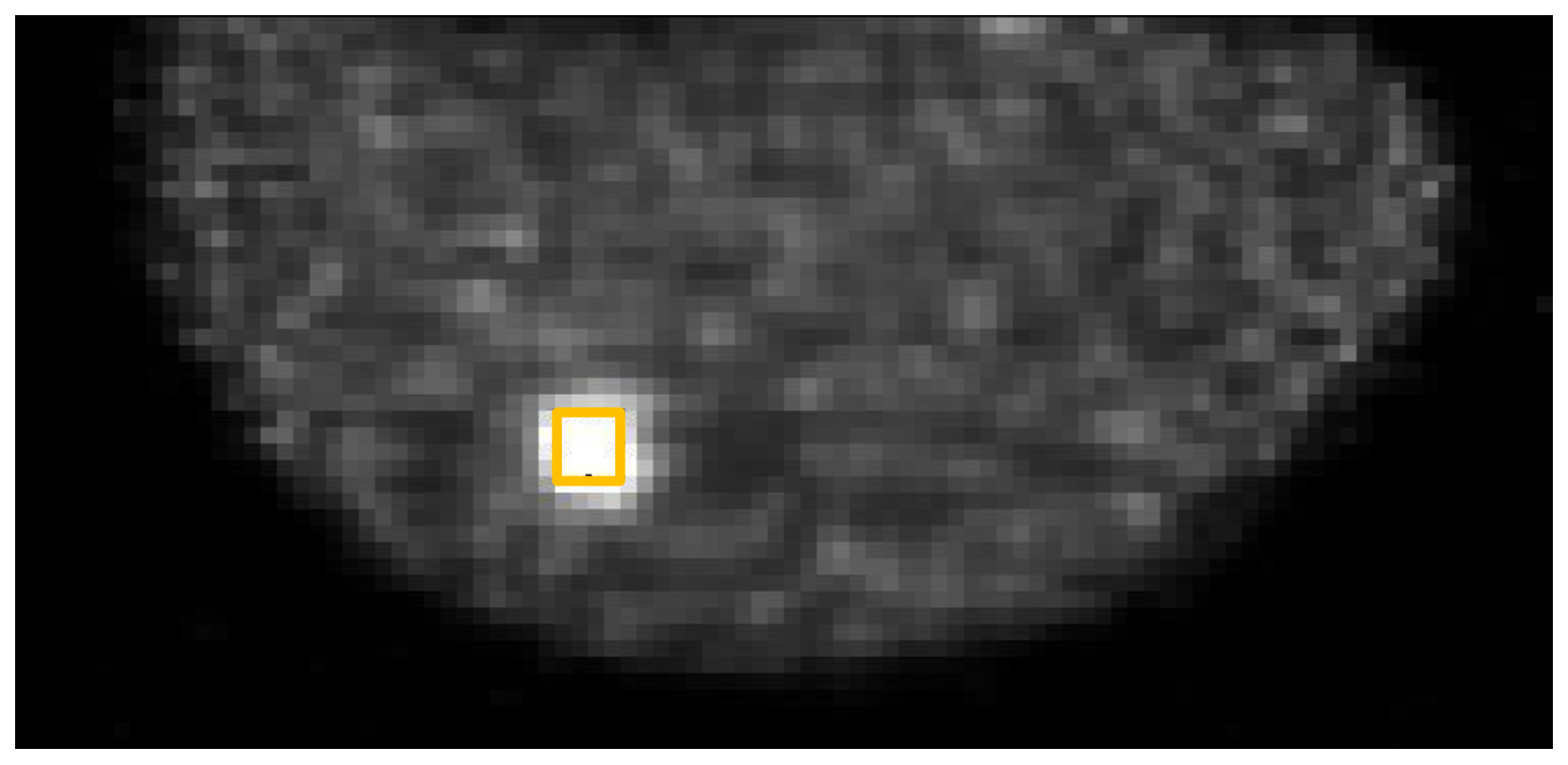

Figure 3-5: In-plane Slice Image Used to Determine Lesion Intensity (parallel to the $x-y$ plane of the gelatin breast phantom)

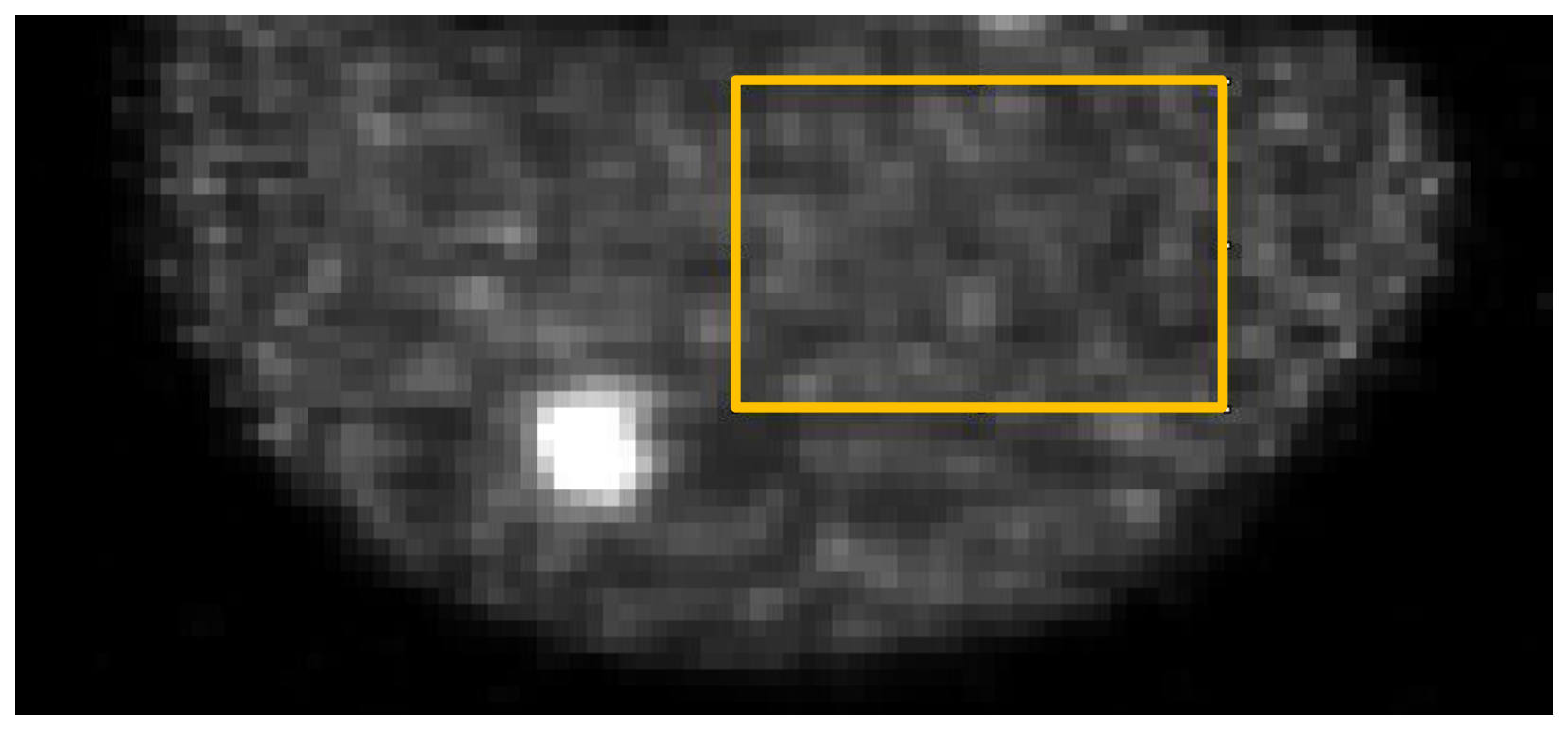

Figure 3-6: In-plane Slice Image Used to Determine Background Intensity (parallel to the $x-y$ plane of the gelatin breast phantom)

Lesion contrast and SNR were subsequently calculated for each trial as follows:

$$
\text { Contrast }=\frac{N_{L}-N_{B}}{N_{B}}
$$




$$
S N R=\frac{N_{L}-N_{B}}{\sigma_{B}}
$$

where $\mathrm{N}_{\mathrm{L}}$ is the average pixel value in the lesion $\mathrm{ROI}, \mathrm{N}_{\mathrm{B}}$ is the average pixel value in the background ROI, and $\sigma_{B}$ is the standard deviation of the pixel values in the background ROI. Of particular importance, the lesion contrast and SNR were calculated for both a "shallow" lesion and a "deep" lesion, located at depths of approximately $20 \mathrm{~mm}$ and $50 \mathrm{~mm}$ below the surface of the phantom, respectively. Image $\mathrm{J}$, a commonly used image processing program, was employed to determine the parameters necessary to evaluate both lesion contrast and SNR. Excel was used to calculate lesion contrast and SNR, and Matlab was used to plot the results which are presented in Figures 3-7 and 3-8. The Matlab source code and Excel files are included in Appendix E. In general, lesion contrast and SNR improvements were observed as graphically depicted below.

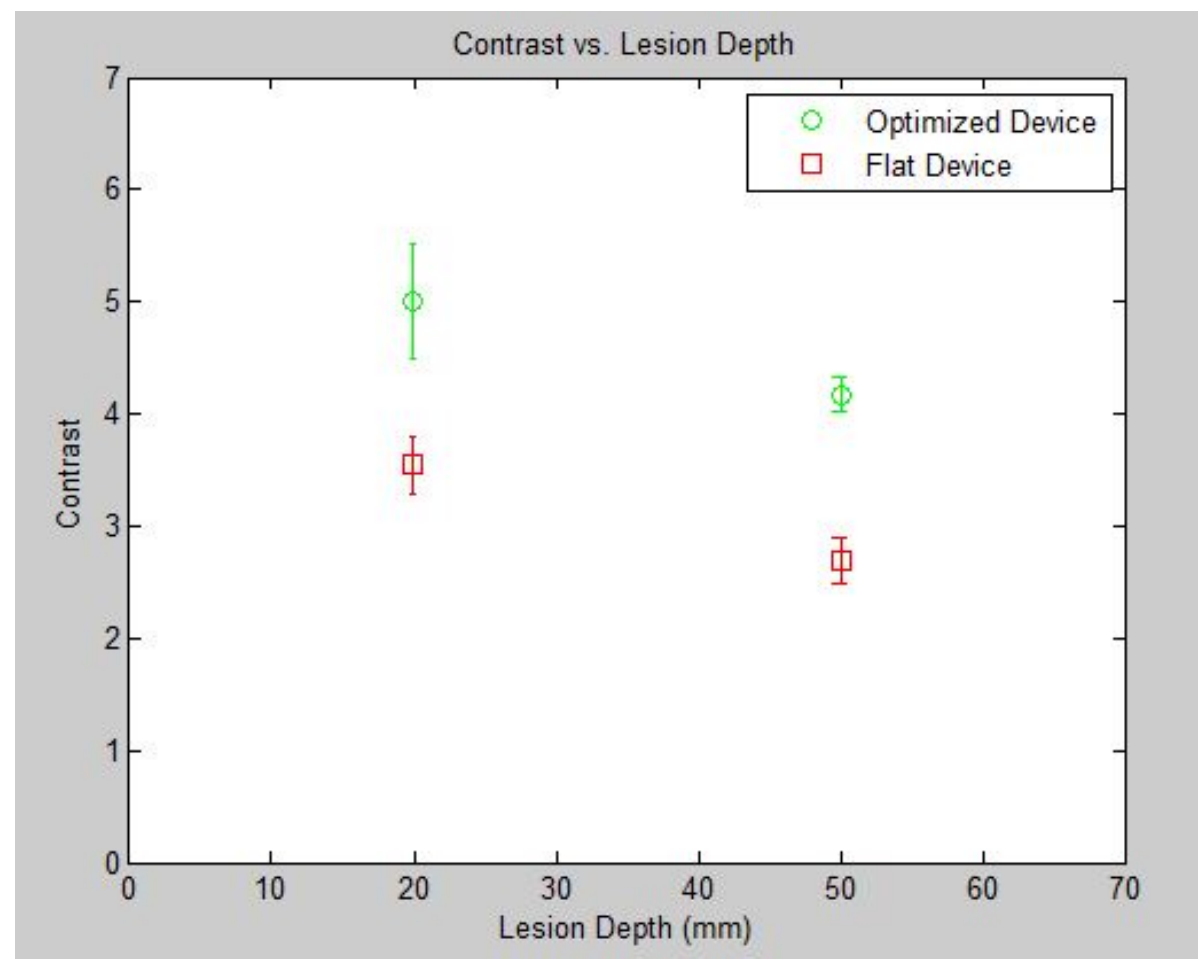

Figure 3-7: Lesion Contrast. Error Bars indicate standard deviation of 5 repeated trials of the experiment. 


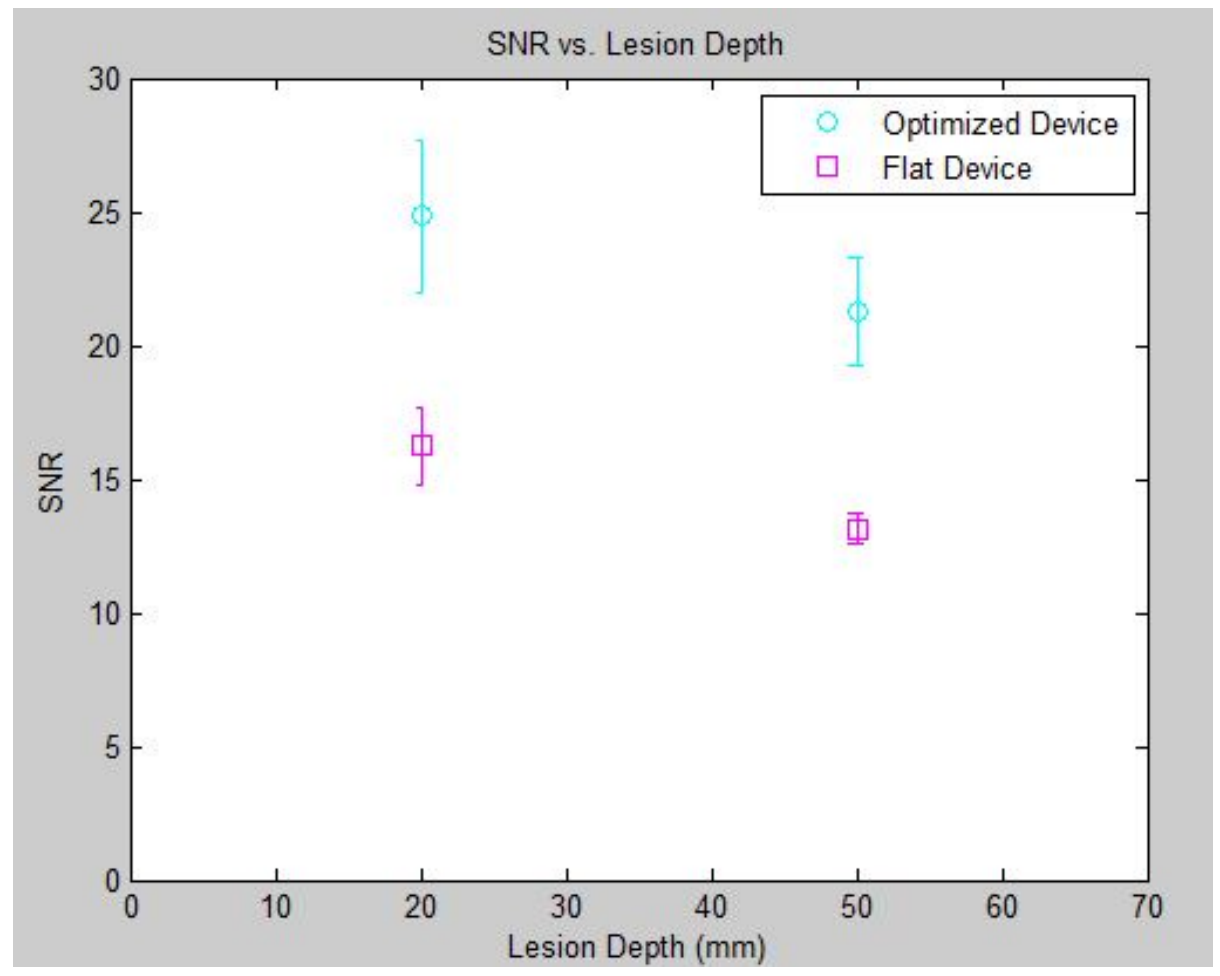

Figure 3-8: SNR. Error Bars indicate standard deviation of 5 repeated trials of the experiment.

\subsection{Discussion}

The "shallow" lesion contrast improved from 3.6 to 5.0, an increase of $41 \%$, while the “deep" lesion contrast improved from 2.7 to 4.2, an increase of 35\%. The "shallow" lesion SNR improved from 16.2 to 24.8, an increase of 53\%, while the "deep" lesion SNR improved from 13.1 to 21.3 , an increase of $38 \%$. If our gamma system was in fact perfect, we would expect the lesion to convolve nicely with a delta function to produce an image that ideally represents the lesion with no additional object blurring or signal intensity reduction. That being said, our system is not perfect, and as such, we expect to see somewhat different results. In reality, the lesion is convolved with a Gaussian function, resulting in a larger, less intense representation of the lesion itself. 
Intuitively, the lesion contrast and SNR improvements observed as a result of these studies are expected. Because the gamma camera detector surface is significantly closer to the lesion when the camera trajectory is dictated by the optimized breast immobilization device (as illustrated in Appendix D), the Gaussian function convolved with the lesion has a smaller standard deviation and full width at half maximum (FWHM). As such, the lesion should appear both brighter and less extensive in the resulting image. We hypothesized that the mean lesion contrast and mean SNR would improve with decreasing radial distance between the gamma camera detector surface and the object (lesion) of interest.

This hypothesis was substantiated by conducting a non-parametric Kruskal-Wallis statistical test to determine whether or not the mean lesion contrast and mean SNR values associated with each trajectory are equal. The Kruskal-Wallis test is merely an extension of the Wilcoxon rank sum test and is frequently used to compare the means between two or more distinct samples. Though our goal is simply to compare two samples, the Kruskal-Wallis test was preferred over the Wilcoxon rank sum test because the Kruskal-Wallis Matlab function outputs both the p-value and a corresponding box and whiskers plot, whereas the Wilcoxon rank sum function does not. The Kruskal-Wallis source code is included in Appendix E.

The Kruskal-Wallis test was chosen in lieu of a much more robust, parametric twosample t-test, or by extension, analysis-of-variance (ANOVA) test, for two main reasons. First, both the two-sample t-test and the ANOVA test assume that "all sample populations are normally distributed" [29]. The Quantile-quantile (QQ) plots presented in Appendix E clearly illustrated that some of the sample data moderately deviates from a straight line, indicating that the data is not normally distributed. The occasional outliers that are observed at the tails of the QQ plots also suggest a deviation from normality. Second, the ANOVA test assumes that "all 
sample populations have equal variance" [29]. Having calculated the variance associated with each sample population as indicated in Appendix E, it is quite obvious that they differ significantly. Consequently, the Kruska-Wallis test was selected for use because it only assumes that all observations are mutually independent and that all samples come from populations with the same continuous distribution [30].

The small p-values presented in Table 3-3 prove that the data is statistically significant and that the null hypothesis can be rejected (p-value $<0.05$ was considered statistically significant). In other words, the mean contrasts associated with both the "shallow" and "deep" lesion differ between the two trajectories, as do the mean SNRs. Furthermore, the box and whiskers plots generated in Matlab and displayed in Figures 3-9 through 3-12, prove not only a difference, but more importantly, an improvement in lesion contrast and SNR, as was expected. These results are encouraging and validate that the prototype design has the potential to help improve image quality as we move forward with our clinical trial studies.

\begin{tabular}{|c|c|c|}
\hline \multirow{2}{*}{ Figure of Merit } & \multicolumn{2}{|c|}{ Lesion Depth } \\
\cline { 2 - 3 } & Shallow (20 $\mathrm{mm})$ & Deep (50 mm) \\
\hline Contrast & 0.009 & 0.009 \\
\hline SNR & 0.009 & 0.009 \\
\hline
\end{tabular}

Table 3-3: P-Values associated with contrast and SNR according to lesion depth. 


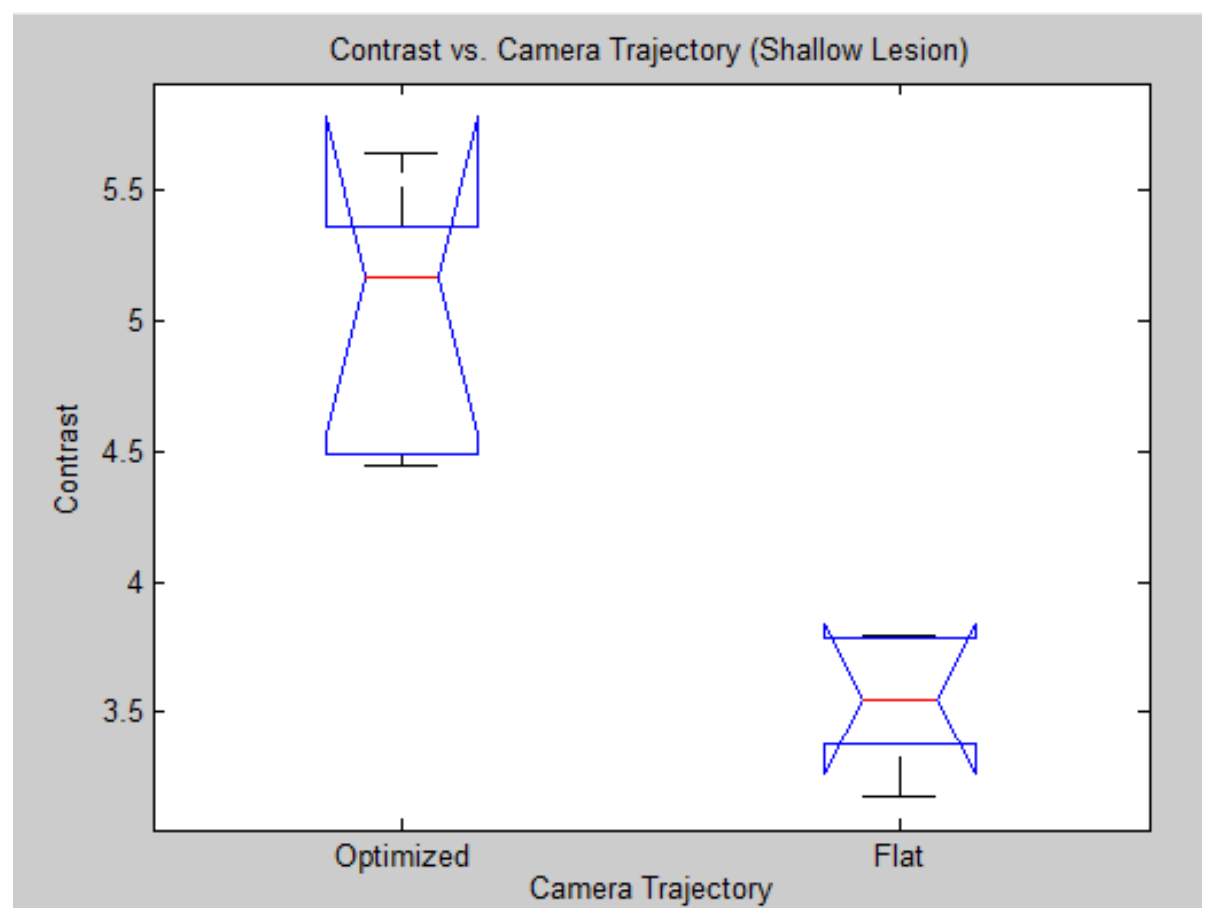

Figure 3-9: Box and whiskers plot comparing contrast (shallow lesion)

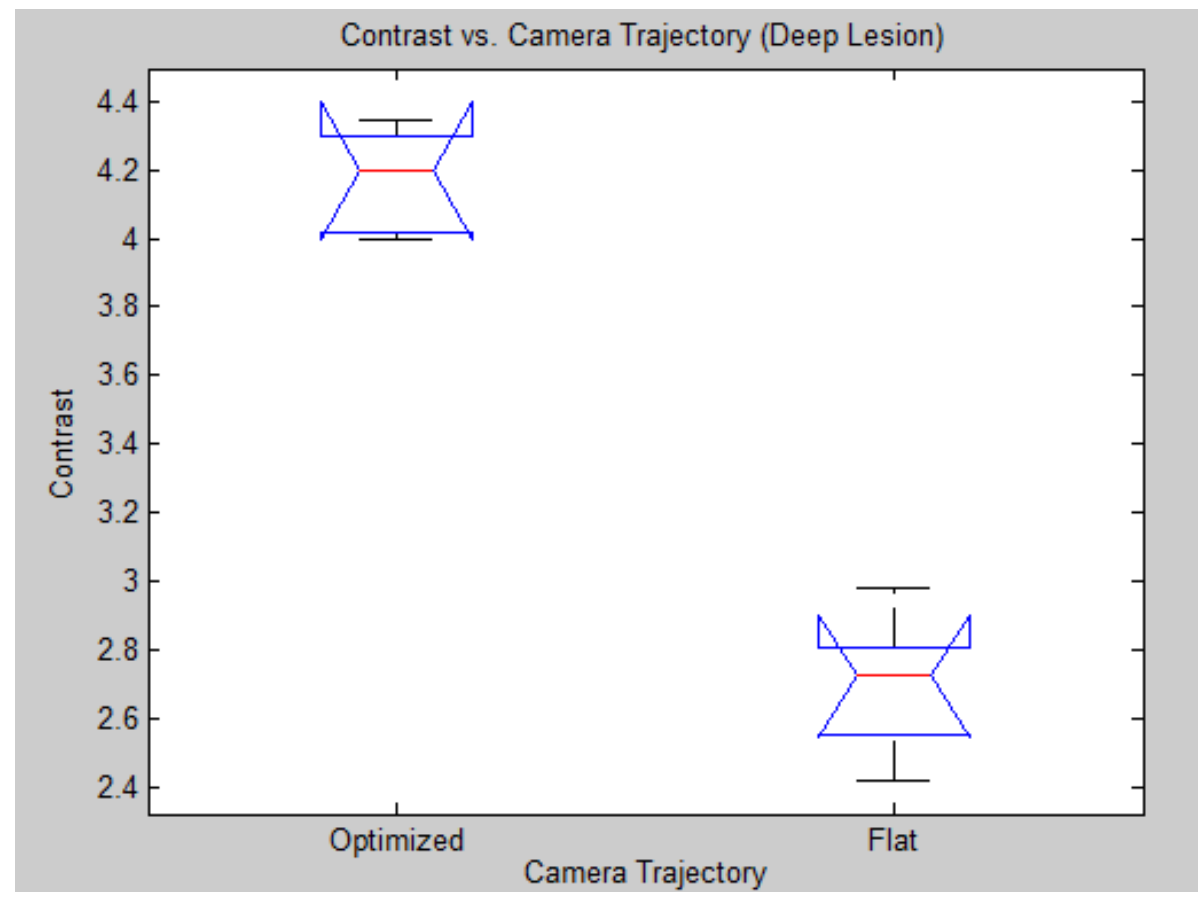

Figure 3-10: Box and whiskers plot comparing contrast (deep lesion) 


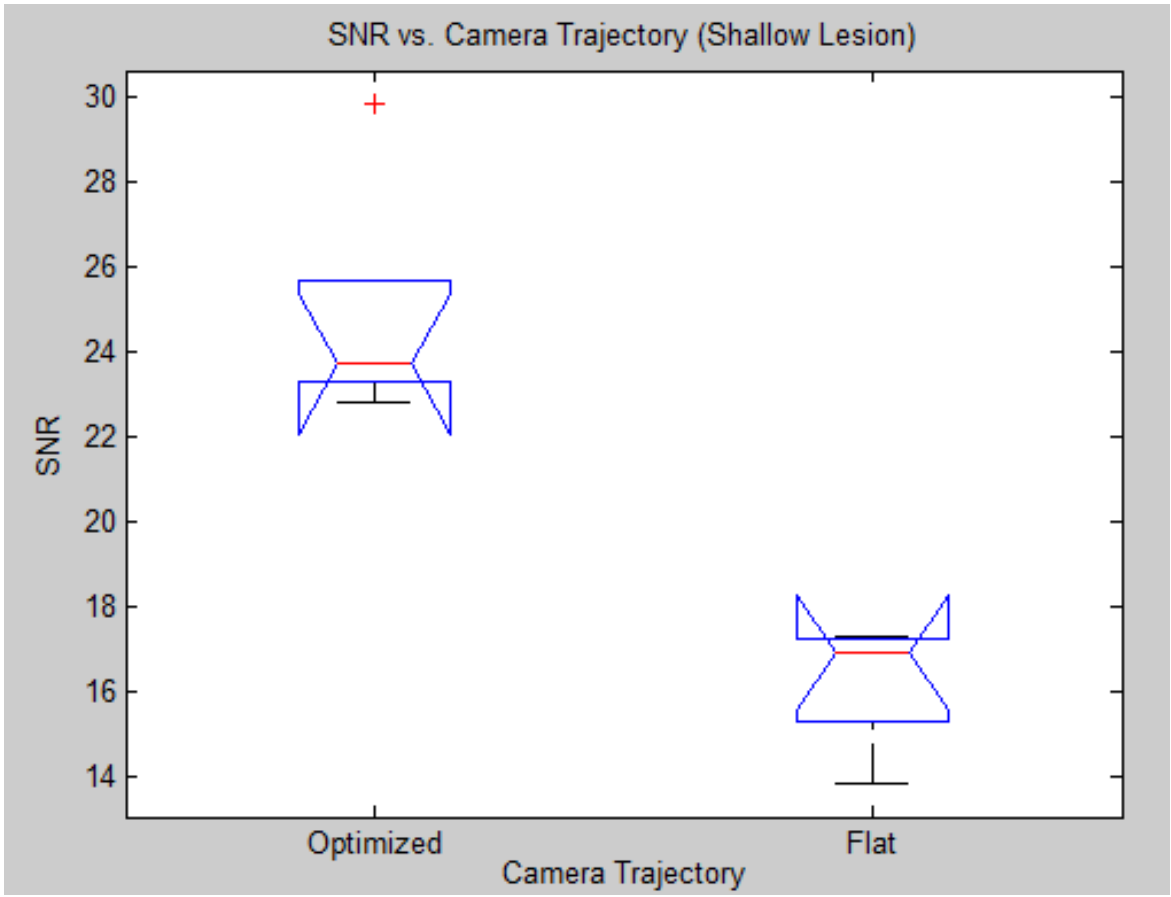

Figure 3-11: Box and whiskers plot comparing SNR (shallow lesion)

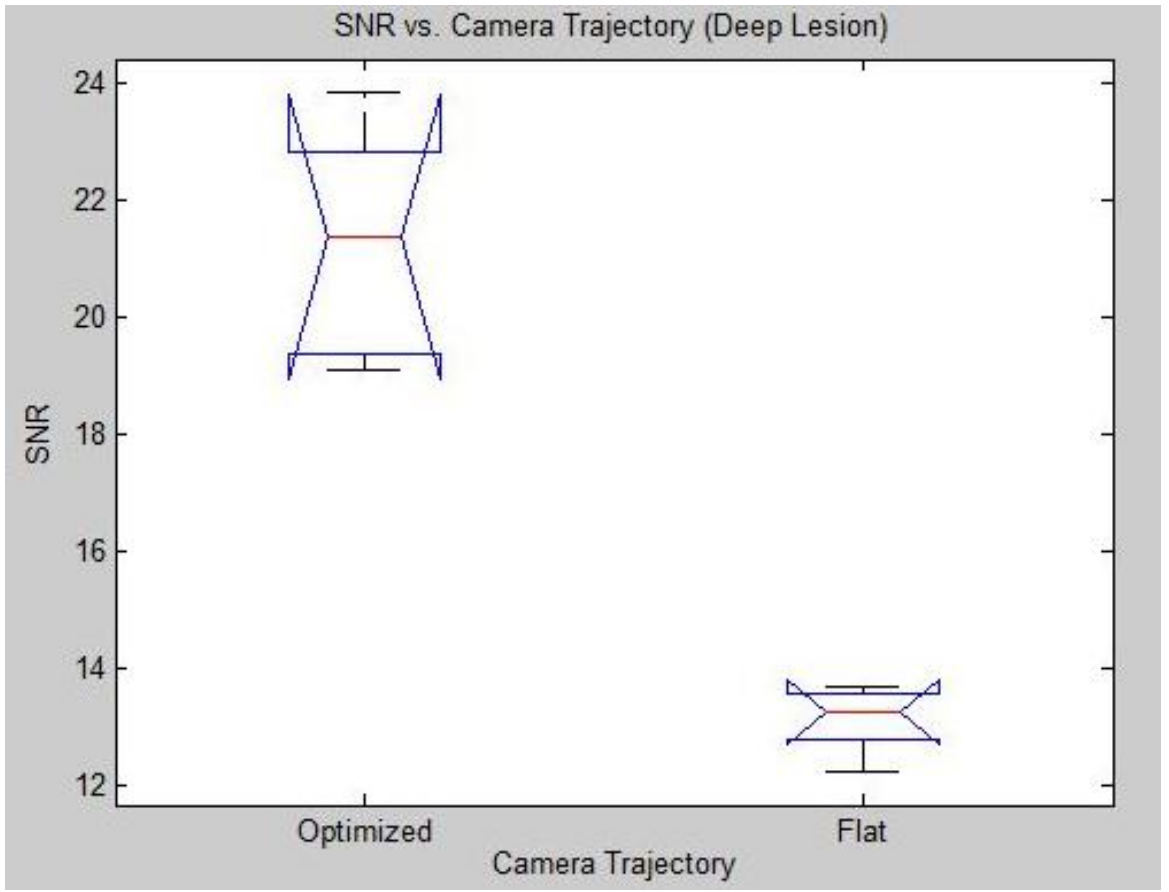

Figure 3-12: Box and whiskers plot comparing SNR (deep lesion)

Note: Box and whiskers plot nomenclature. Red central line denotes median data point, top black line denotes maximum data point, bottom black line denotes minimum data point, top blue line denotes $75^{\text {th }}$ percentile (calculated using the quantile function $\&$ linear interpolation), bottom blue line denotes $25^{\text {th }}$ percentile (also calculated using the quantile function and linear interpolation), and red + sign indicates outliers 


\section{PROTOTYPE EVALUATION AND REDESIGN}

Having assessed the validity of the optimized breast immobilization device experimentally using a gelatin breast phantom, we recognized the importance of evaluating the prototype in a research setting with real human breasts. As such, we integrated the prototype into the DMT gantry and recruited two women with very different breast sizes to participate in an investigative study aimed at understanding how well the prototype works to compress the breast. The strengths and weaknesses of the optimized device were revealed as a result of this study, ultimately driving future alternative design recommendations. The volunteers were not exposed to radiation during this investigation, as the goal of the study was to evaluate the prototype's ability to fully compress the breast, irrespective of size, shape, and elasticity. 


\subsection{Optimized Breast Immobilization Device Design Limitations}

A pregnancy belly cast kit (Figure 4-1) was used to evaluate the effectiveness of the optimized breast immobilization device in terms of its ability to compress breasts of varying sizes. Though slightly unorthodox, this kit allowed us to create a mold of the breast during compression. First, the gantry arm was positioned at approximately $+40^{\circ}$ so that compression could be applied from the medial side of the breast to the lateral side of the breast at an oblique angle (MLO view). The MLO view was used to acquire projection data during the pilot DMT study. After positioning the gantry arm, we applied wet

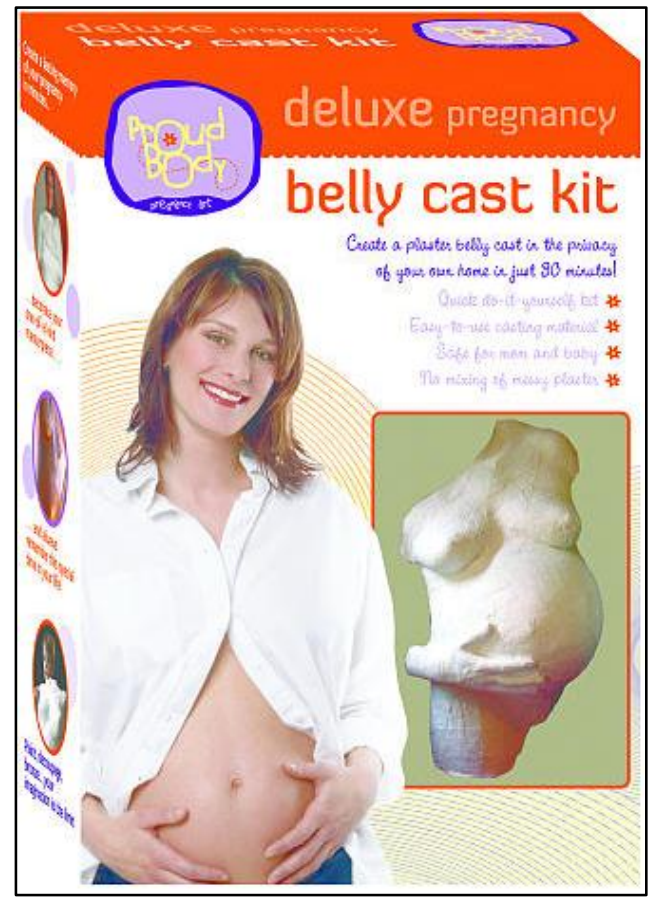

Figure 4-1: Pregnancy belly cast kit plaster from the belly cast kit to the breast of interest. A radiation technologist then positioned the breast inside the optimized breast immobilization device and applied compressive force using the mechanical crank system. The plaster dried into a mold of the compressed breast as shown in figure 4-2.

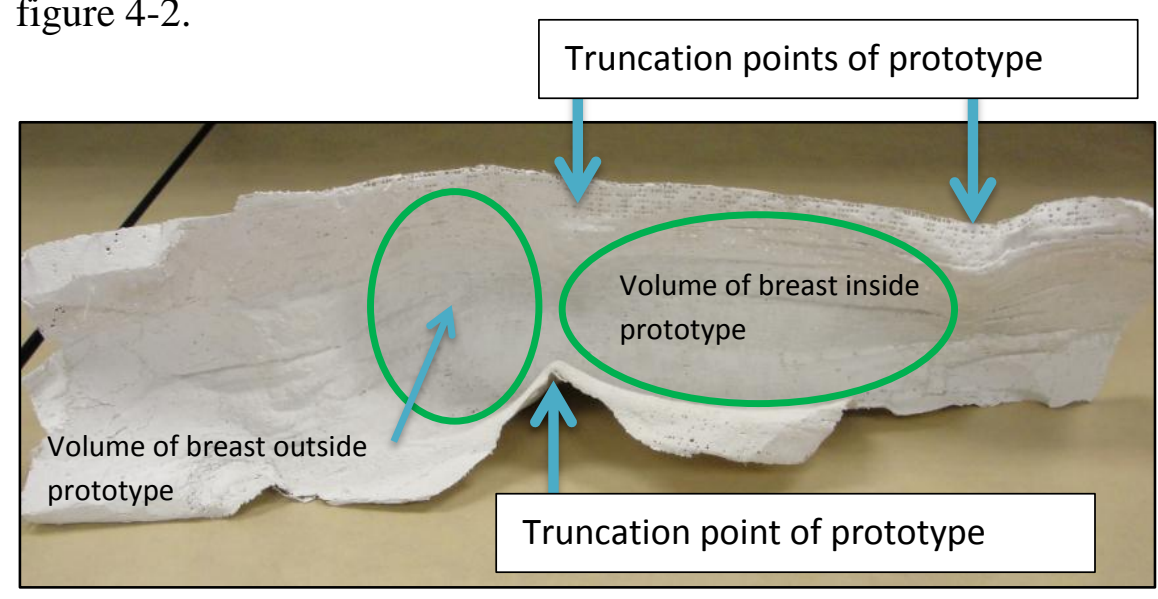

Figure 4-2: Mold of breast compressed with optimized immobilization device 
While one of the participants in this study had a smaller breast size, equating to a 34A bra size, the other had a larger breast size, equating to a 32D bra size. Though these breast sizes differ significantly, the results of the study were qualitatively similar for both volunteers. Each mold showed that a certain percentage of breast tissue was not being compressed by the optimized breast immobilization device. The radiation technologist was able to position the breasts such that the pectoral muscle was held securely in place; however, the medial breast tissue was compromised. As evident in Figure 4-2, the prototype pinched the medial tissue, segmenting it into two distinct fractions, causing notable pain and discomfort. Simply put, the optimized breast immobilization device is not wide enough to accommodate all of the breast tissue, even for relatively small breast sizes.

\subsection{Support System Limitations}

Unfortunately, the support system that integrates the optimized breast immobilization device into the DMT gantry is not without design flaws as well. Delrin, a very strong though highly-attenuating polymer, was the material originally chosen for the side pieces of the support structure. However, we modified the design, and instead suggested the use of carbon-fiber. Carbon-fiber, also well known for its strength, is significantly less radio-opaque than Delrin. As such, we were hoping that it would not be as visible in the acquired x-ray projection images. Disappointingly, the rigid carbon-fiber supports were very obvious in the projection images, at different locations across the angular range employed. The carbon-fiber could potentially obscure small lesions or micro-calcifications, tiny speckles that can sometimes appear in a structural image and are, at times, indicative of disease presence. The support structures themselves would be very difficult to correct out of the x-ray image, and as such, alternative attachment methods were considered. 


\subsection{Optimized Breast Immobilization Device Alternative Design}

An area analysis was completed to justify our experimental observations and help quantify a wider, more inclusive prototype to avoid "pinching" during future clinical trial studies. Recalling equation (22), we were able to calculate the total area associated with the truncated optimized breast immobilization device.

$$
A_{2}=4 *\left[\frac{a b}{2} \sin ^{-1}\left(\frac{w}{2 a}\right)+\frac{b w}{4}\left(\sqrt{1-\frac{w^{2}}{4 a^{2}}}\right)-\left(\Delta *\left(\frac{w}{2}\right)\right)\right]
$$

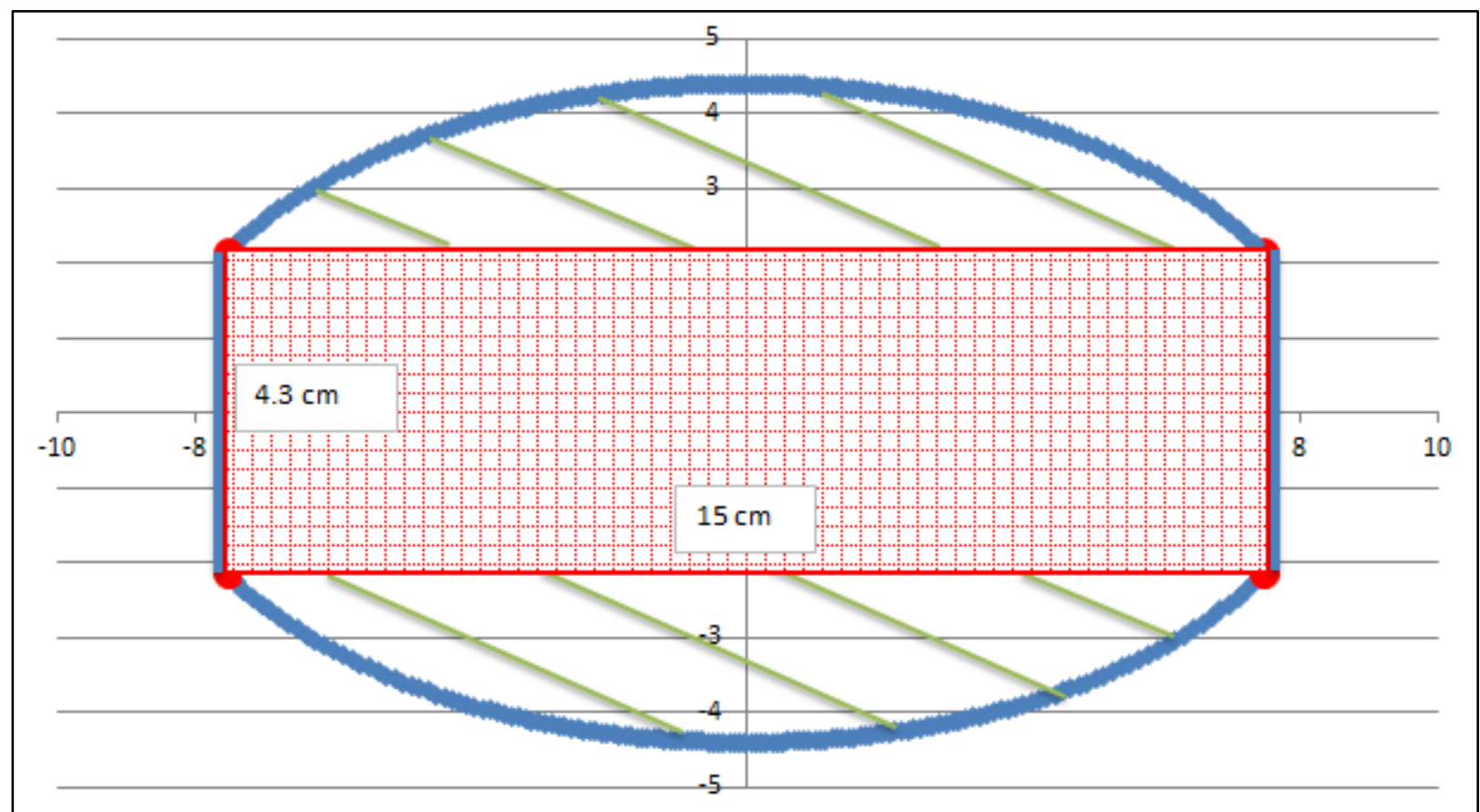

Figure 4-3: Cross-sectional area of truncated optimized breast immobilization device

$\mathrm{A}_{2}$, the area enclosed by the blue lines in Figure 4-3, equates to $112.5 \mathrm{~cm}^{2}$. Upon further consideration of Figure 4-3, we determined $A_{3}$, the area of the red checkered rectangle region and $A_{1}$, the area of the two regions defined by the green diagonal lines. $A_{3}=64.6 \mathrm{~cm}^{2}$, while $A_{1}$ $=\mathrm{A}_{2}-\mathrm{A}_{3}=48 \mathrm{~cm}^{2}$. The value of $\mathrm{A}_{1}$ defines the cross-sectional area of a breast that is compressed such that the truncation points of the compression paddle touch the truncation points 
of the breast support, assuming that there is visible contact between breast tissue at the chest wall and each point defining the edge of the optimized breast immobilization device.

Recalling equation (6), the area of an ellipse characterized by our $1100 \mathrm{~mL}$ gelatin breast phantom was determined as follows:

$$
A_{4}=\pi * a * b=\pi * 8.6 * 4.4=118.9 \mathrm{~cm}^{2}
$$

Clearly, the value of $A_{1}$ is significantly less than the value of $A_{4}\left(48 \mathrm{~cm}^{2}<118.9 \mathrm{~cm}^{2}\right)$. Though the separation distance between the truncation points of the curved compression paddle and those of the breast support is dictated by both the volume and elasticity of the breast tissue, these area comparison results verify that we cannot expect to contain all of the tissue associated with a large breast within the constraints of the current optimized breast immobilization device.

These insights substantiate the need to propose a wider version of the original design, with the understanding that the recommended alternative must have an area (defined by the green diagonal line region) greater than or equal to $\mathrm{A}_{4}$, while still maintaining the same semi-major to semi-minor axis ratio $(a / b)$ as the prototype. The $a / b$ ratio associated with the prototype is equal to 1.95 . We decided to use the width of the current flat paddle (9.5 inches) as the starting point for determining the new truncated width of the adapted design, as it successfully immobilized even the largest breasts that were observed during the pilot DMT study. If the total truncated width $\left(w_{\text {scaled }}\right)$ of the suggested alternative were to equal 9.5 inches $(24.13 \mathrm{~cm})$, instead of 5.9 inches, then $w_{\text {scaled }} / 2$ would equal 4.75 inches or $12.065 \mathrm{~cm}$. Prior to calculating the semi-major and semi-minor axes that correspond to $w_{\text {scaled }}=9.5$ inches $=24.13 \mathrm{~cm}$, the ratio of $w / 2$ (the halfwidth of the original prototype) to the semi-major axis of the original design had to be computed. This ratio is equal to 0.87 . The semi-major axis of the scaled design is then determined by 
dividing $w_{\text {scaled }} / 2$ by 0.87 . The new semi-major axis $\left(a_{\text {scaled }}\right)$ is equal to 5.45 inches or $13.85 \mathrm{~cm}$. The new semi-minor axis ( $b_{\text {scaled }}$ ) can then be determined by dividing $a_{\text {scaled }}$ by 1.95 . The new semi-minor axis ( $\left.b_{\text {scaled }}\right)$ is equal to 2.79 inches or $7.08 \mathrm{~cm}$.

The area associated with the truncated alternative design, $\mathrm{A}_{2 \text { scaled, }}$, is then calculated as previously shown using equation (22). $A_{2 s c a l e d}=291.46 \mathrm{~cm}^{2}$. In keeping with Figure 4-3, $A_{3 \text { scaled }}$, the new area of the red-checkered region, equals $167.36 \mathrm{~cm}^{2}$. Finally, $\mathrm{A}_{1 \text { scaled, }}$, the new area defined by the green diagonal lines, is equal to $124.09 \mathrm{~cm}^{2}$. Clearly, the value of $\mathrm{A}_{1 \text { scaled }}$ is slightly greater than $\mathrm{A}_{4}$, the area of an ellipse defined by the $1100 \mathrm{~mL}$ gelatin breast phantom. In addition, $a_{\text {scaled }} / b_{\text {scaled }}=a / b=1.95$. Having met the suggested criterion, it appears that the scaled version of the prototype should successfully enclose all of the tissue when the cross-sectional area of the compressed breast is equal to that of the $1100 \mathrm{~mL}$ gelatin breast phantom, even if the truncation points of the scaled device are touching. The overall dimensions of the scaled prototype are 7.75 inches long x 9.50 inches wide x 1.78 inches high. A detailed drawing of the enlarged version of the optimized breast immobilization device is included in Appendix F.

The scaled version of the optimized breast immobilization device is designed to comfortably compress and immobilize rather large breasts; however, the ability to accomplish this task greatly depends on the elasticity of the tissue itself. Breast tissue that is very elastic has a low Young's modulus, while breast tissue that is not very elastic, has a much higher Young's modulus. Should a relatively large breast have a correspondingly low Young's modulus, it may prove difficult to immobilize, as we may experience a "zero force scenario" where the truncation points of the scaled device are touching without forcefully compressing or immobilizing the breast tissue. This scenario, in conjunction with the need to immobilize a wide range of breast sizes, motivated us to design yet another prototype with a total truncated width larger than that of 
the original design, yet smaller than that of the scaled alternative. The width of the mid-size design was chosen to be 7.50 inches or $19.05 \mathrm{~cm}$. The aforementioned scaling ratios were again used to determine the semi-major and semi-minor axes of the mid-size design. The corresponding semi-major axis, $a_{\text {mid }}$, is equal to 4.30 inches or $10.93 \mathrm{~cm}$, while the corresponding semi-minor axis, $b_{\text {mid }}$, is equal to 2.20 inches or $5.59 \mathrm{~cm}$. The overall dimensions of the mid-size device are 7.75 inches long x 7.50 inches wide x 1.50 inches high. A detailed drawing is included in Appendix F.

\subsection{Support System Alternative Design}

A multitude of structural support system design alternatives were discussed as the project progressed. One alternative given serious consideration was an adaptation of the current support structure design, illustrated in Figure

2-11. The support system limitations outlined in section 4.2 emphasize the importance of maintaining a uniform, unobstructed background image, especially with respect to the location of the breast within a given projection view. As a result, our first inclination was to modify the current structure

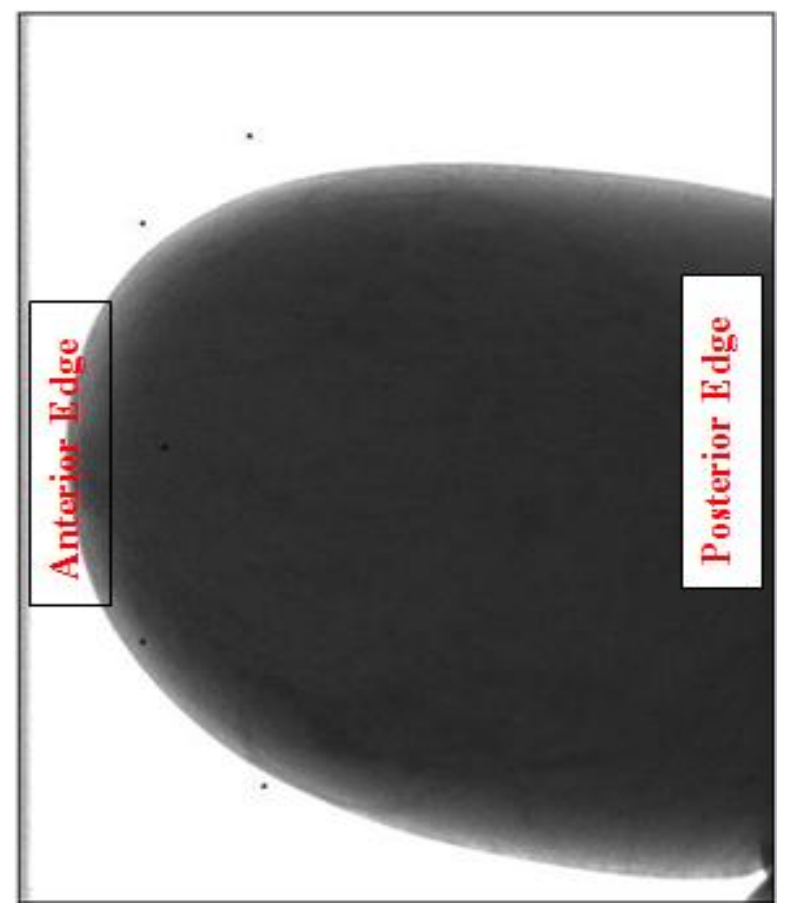

Figure 4-4: Example acquired image of a large breast by cutting off the carbon-fiber side pieces such that they extend approximately one third of the way down the stabilizing lips of the immobilization device from the anterior end. This alteration would improve upon the current design and ensure that the carbon-fiber component parts would 
only appear at the anterior edge of the resulting image. On average, the breasts imaged thus far as part of the pilot DMT study occupied $55 \%$ of the total length of the image in the posterior to anterior direction. Figure 4-4 illustrates that a select few extended almost the entire length of the image in the posterior to anterior direction. As a result, this design alternative was ultimately abandoned in favor of a much cleaner approach.

Of the approaches considered, the design presented in Figure 4-5 was favored for two distinct reasons. First, all of the component parts are positioned outside of the field of view. Thus, any small lesions or micro-calcifications that may be present will not be obscured by the side supports. Second, the design is easily integrated into the lead screw crank system structure that was previously developed to assimilate the original prototype. The grey component part shown in Figure 4-5 is made of aluminum, while the green component part is made of

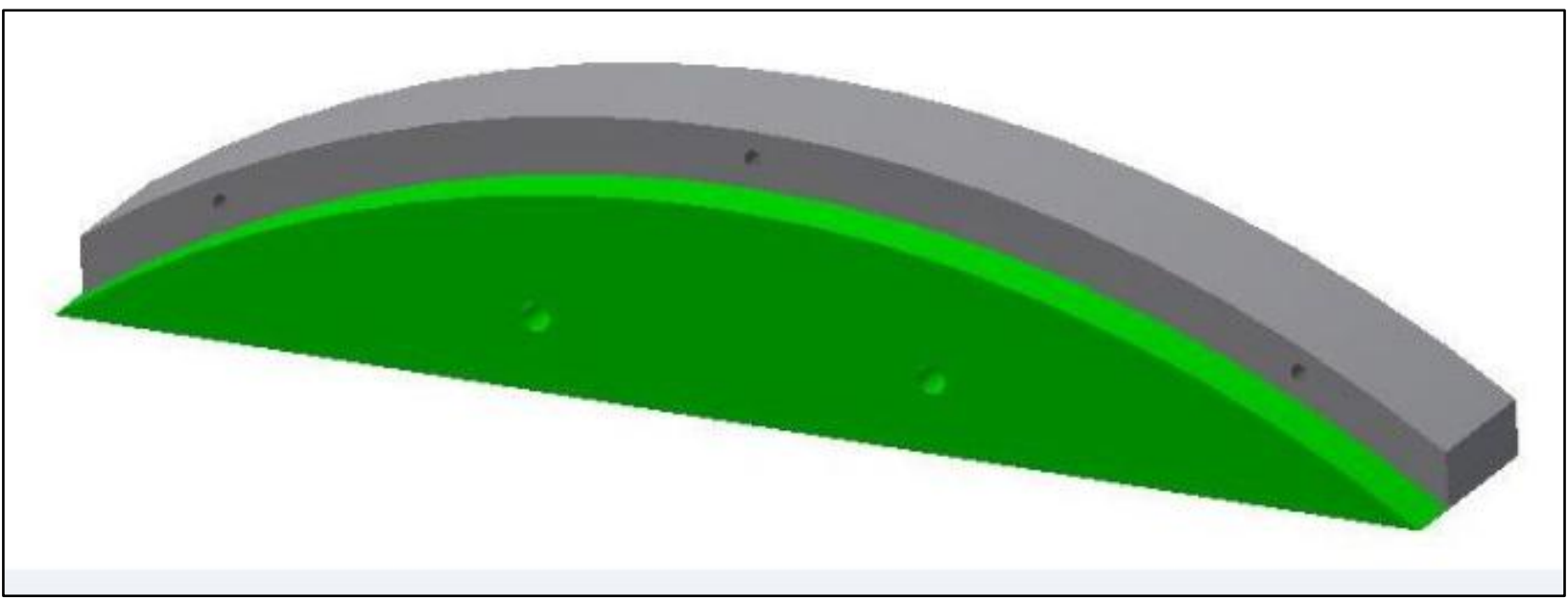

Figure 4-5: Redesigned support system structure

polycarbonate. The aluminum piece is ultimately affixed to the linear bearings that translate the immobilization device up and down in the z-direction. The polycarbonate piece is epoxied along the arc to the underside of the breast immobilization device and subsequently screwed into its aluminum counterpart. For added security, the breast immobilization device is also fastened to the aluminum piece with nylon screws as was shown in Figure 2-11. Three support system 
structures will be tailor-made for each of the aforementioned prototype designs to ensure both efficiency and functionality. Detailed drawings for the mid-size support structure, as well as the large support structure, are included in Appendix F. 


\section{DMT SCANNER OPTIMIZATION}

Though the curved nature of the optimized breast immobilization device permits wide angle acquisition, a number of hardware assembly restrictions, specific to the DMT scanner, hinder our ability to obtain projection views at wide angles. These limitations must be overcome to realistically attain the image quality improvements experimentally determined in Chapter 3. As such, a number of hardware component parts will need to be modified or replaced to accomplish the technical goals set forth at the onset of the project. For example, the aluminum arm shown in Figure 5-1 will need to be modified, as will its counterpart, which is mirrored about the z-axis of the DMT scanner. In addition, the x-ray detector supports shown in Figure 52 will need to be completely redesigned and ultimately replaced. These hardware adjustments are discussed in detail in the sections to follow, as are the motivating factors that compelled us to invoke change. 


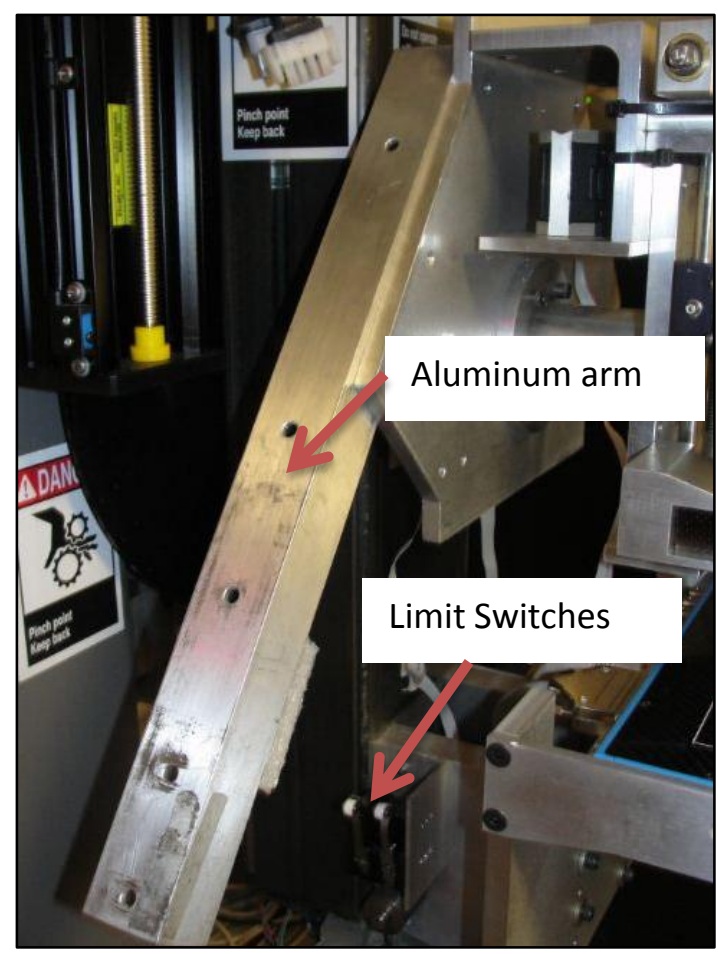

Figure 5-1: Photograph of aluminum arm

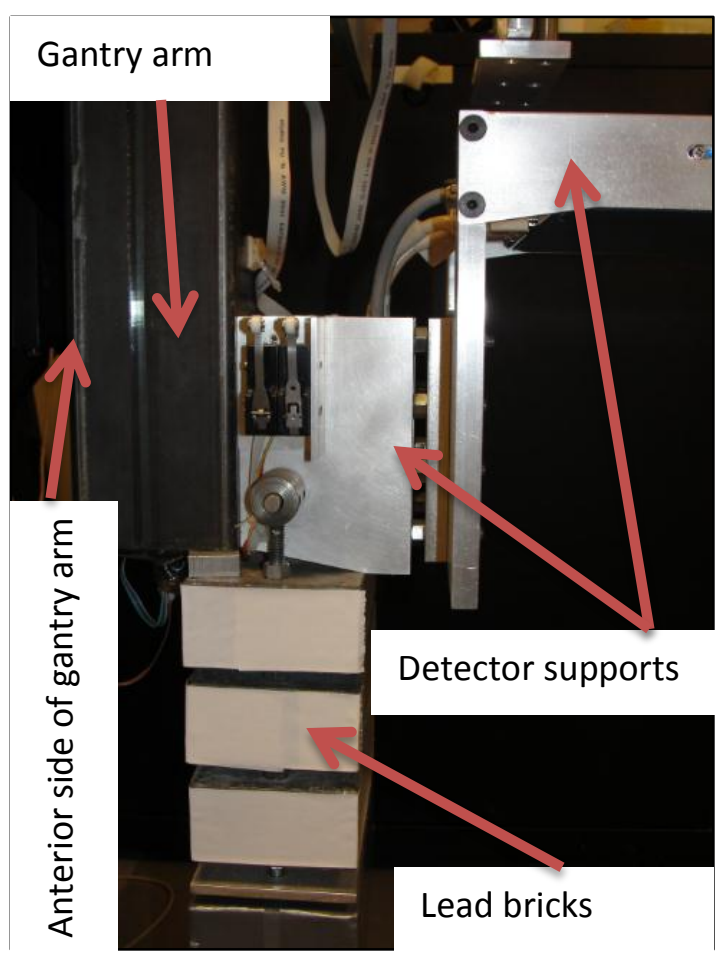

Figure 5-2: Photograph of X-ray detector housing

\subsection{Aluminum Arm Deficiencies}

The aluminum arm depicted in Figure 5-1 serves two primary purposes. First, it provides a place for the patient to rest her hand during the scan. Prior to positioning the breast, the radiation technologist places the patient's arm (corresponding to the breast of interest) in the "up position," resting her hand towards the top of the aluminum piece. Doing so permits easy access to the pectoral muscle and ensures that it will remain securely in place once compressed.

Second, the aluminum arm serves as an attachment point for the aluminum stomach guard that shields the patient's stomach from moving parts (such as the x-ray detector) when the machine is in motion. Unfortunately, when the optimized breast immobilization device is integrated into the DMT scanner, these aluminum piece parts only allow the gantry arm to rotate $+/-36^{\circ}$ away from the direction of compression. Should the rotation angle exceed $+/-36^{\circ}$, the aluminum arms will collide with the limit switches attached to the x-ray detector housing, which serve as a safety 
mechanism to prevent over-rotation. The angle range dictated by the aluminum arms is significantly larger than the angle range used during the pilot DMT study. However, it proves undesirable when considering the angle range (+/-67.5 $)$ employed experimentally.

\subsection{Aluminum Arm Modifications}

In order to permit wide angle acquisition, the aluminum arms will need to be cut off 10.125 inches from the top, so that as the DMT scanner rotates, the lowest point of the aluminum arm clears the horizontal plane tangent to the top of the x-ray detector housing. From a functional standpoint, this retrofit still provides the patient with a resting place for her hand; however, it eliminates the aluminum arm's ability to serve as an attachment point for the stomach guard. Consequently, the stomach guard will need to be modified accordingly. In addition, the limit switches will need to be moved to an appropriate location once an acquisition angle range has been solidified. These systematic modifications, though critical to the success of the optimized breast immobilization device, will ultimately be implemented as "future work" in keeping with the concluding remarks of Chapter 6.

\subsection{X-ray Detector Support Deficiencies}

The x-ray detector support, shown in Figure 5-2, attaches the x-ray detector to the DMT

gantry arm. When the optimized breast

immobilization device is used to compress the breast and the aluminum arms are retrofitted as previously suggested, the x-ray detector, in theory, should be free to rotate about the breast. However, because the

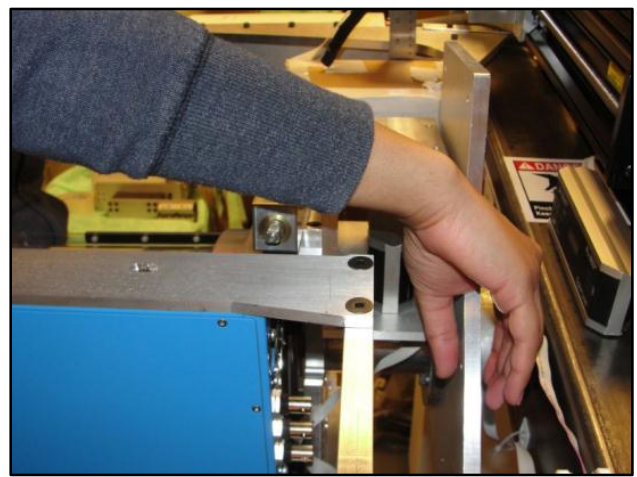

Figure 5-3: Photograph of $x$-ray detector \& patient arm collision 
patient's arm is placed in the "up position," as illustrated in Figure 5-3, the x-ray detector will eventually collide with it, preventing both the x-ray and gamma ray detectors from obtaining wide angle projection views at positive angles if the right breast is compressed, and at negative angles if the left breast is compressed. As such, we then had to consider the possibility of acquiring both XBT and MBIT projection images across an asymmetrical angle range. Though we chose to rotate the DMT scanner symmetrically about the AOR during the pilot DMT studies and the gelatin breast phantom experiments, nothing precludes us from considering an asymmetrical angle range as we move forward with the progression of the design and begin to look towards patient accrual.

The maximum attainable XBT acquisition angle (in one direction) will be dictated by the point at which the x-ray detector collides with the patient's arm; while, the maximum attainable XBT acquisition angle (in the opposite direction) will be dictated by the point at which the patient's arm begins to appear in the projection image. The total XBT and MBIT acquisition angle ranges are driven by the ergonomics of the DMT scanner in relation to patient positioning. The total MBIT acquisition angle range will differ from that of $\mathrm{x}$-ray breast tomosynthesis, as the $\mathrm{x}$-ray detector is not needed to acquire gamma ray projection images. Therefore, after the XBT projection views are acquired, the x-ray detector can be moved out of the way to permit wide angle MBIT acquisition.

\subsection{X-ray Detector Support Modifications}

Two different approaches were explored to accomplish wide angle MBIT acquisition. Initially, we considered removing the x-ray detector from the DMT gantry upon completion of the x-ray portion of the scan. This approach was ultimately abandoned for three reasons. First, it 
would be difficult to accomplish in a timely manner. Second, it would prove problematic in terms of accessibility, as the patient is standing (or sitting) directly in front of the x-ray detector, thus restricting access to it. Third, it would be very difficult to ensure that the $\mathrm{x}$-ray detector was repositioned in the exact same place with each successive patient.

As a result, we chose to redesign the $\mathrm{x}$-ray detector support such that the entire subassembly, including the $\mathrm{x}$-ray detector itself, can be rotated $90^{\circ}$ from its initial position parallel to the floor, to an alternative position, parallel to the DMT gantry arm. Two, low-profile mounted ball bearings, manufactured by Turner Mounted Bearings (model number UCTB-2018), will serve as the axis-of-rotation for the subassembly. These ball bearings will be mirrored about the DMT gantry arm and mounted to identical aluminum plates on opposite sides of the scanner. They will also be fitted with aluminum cylinders that will be welded to the aluminum side arms of the x-ray detector. Though similar in size to their predecessors, these side arms will extend further in the posterior-to-anterior direction such that they intersect the axis-of-rotation dictated by the position of the ball bearings. The side arms, and by association the x-ray detector, will also be held securely in place by two close-fitting aluminum blocks wedged between the end of their respective anterior edges and the aluminum plates that will be mounted on opposite sides of the scanner. These aluminum blocks will ensure that the x-ray detector is returned to the exact same position with each successive scan. The subassembly has been built per the drawings presented in Appendix G; however, it will be integrated into the DMT scanner as "future work" in conjunction with the retrofits discussed in Section 5.2.

In order to accomplish the x-ray detector support modifications, the lead brick weights that hang from it, as shown in Figure 5-2, will have to be relocated. These lead bricks serve as a counterbalance to the $\mathrm{x}$-ray tube, which weighs 82 pounds. By moving these bricks and 
attaching them from the anterior side of the gantry arm, we can ensure that they continue to offset the weight of the x-ray tube and clear the plane of the x-ray detector when it parallels the gantry arm, having been hinged down $90^{\circ}$. The subassembly that has been designed to accomplish this task consists of two aluminum piece parts, the smaller of which will attach directly to the anterior side of the gantry arm, the larger of which will anchor the weights. Much like the x-ray detector housing, the subassembly has been built per the drawings in Appendix G; however, it too will be integrated into the DMT scanner as "future work." 


\section{SUMMARY, CONCLUSIONS, AND FUTURE DIRECTION}

\subsection{Summary}

The overall goal of this project was to develop an innovative breast immobilization device that can be integrated into the DMT scanner to improve image quality as well as patient comfort. We hypothesized that MBIT image quality could be improved by reducing the radial distance between the gamma camera and the breast at large viewing angles away from the zero degree view. More specifically, we predicted that the image contrast and signal-to-noise ratio (SNR) would improve as the radial distance was reduced. We also hypothesized that compressing the breast in a more natural configuration would increase the level of patient comfort during a scan.

Preliminary experimental studies showed that the optimized breast immobilization device that we created is capable of improving both image contrast and signal-to-noise ratio (SNR) as 
compared to standard, flat paddle compression. Notable improvement was realized, independent of lesion depth. The "shallow" lesion contrast improved from 3.6 to 5.0, an increase of $41 \%$, while the "deep" lesion contrast improved from 2.7 to 4.2 , an increase of $35 \%$. The "shallow" lesion SNR improved from 16.2 to 24.8 , an increase of 53\%, while the "deep" lesion SNR improved from 13.1 to 21.3 , an increase of $38 \%$.

A prototype was built and integrated into the DMT scanner. The prototype is comprised of an elliptical compression paddle and breast support, both of which are vacuum formed from 0.125 inch thick polycarbonate. The overall dimensions of the device are 7.75 inches long x 5.91 inches wide x 1.26 inches high. Rigidity was added into the design by including a 0.375 inch high lip along the perimeter edge of both component parts. The prototype's ability to securely and comfortably compress a breast was evaluated using a body cast molding kit, which demonstrated that the device was not wide enough to accommodate the medial breast tissue, pinching it into two well-defined segments. Accordingly, we are in the process of fabricating similar immobilization devices with a range of sizes to be evaluated for their ability to comfortably accommodate a broad range of breast sizes, while still providing improved image contrast and SNR.

\subsection{Conclusions}

In conclusion, the optimized breast immobilization device, when sized appropriately to accommodate all of the breast tissue, allows for wide-angle acquisition, improved image quality and patient comfort. The total angular range employed for both XBT and MBIT will be determined based on DMT scanner system modifications and patient positioning. Actual image quality enhancement will need to be evaluated during future clinical trial case studies, once the 
angular range (specific to each modality) has been solidified. Moreover, patient comfort will also need to be assessed as we begin to look towards accruing human subjects for impending clinical trial studies.

\subsection{Future Direction}

Initial evaluation of the optimized breast immobilization device has proven encouraging; however, future assessment is necessary to further quantify improvement both from a technical and non-technical standpoint. As indicated in chapter 5, a number of DMT system modifications will need to be implemented prior to accruing patients, so as to assess the validity of the design using real human breasts. These system modifications include, retrofitting the aluminum arm that attaches to the DMT gantry, relocating the lead brick weights that counter-balance the x-ray tube, integrating the redesigned x-ray detector support mechanism into the DMT gantry, and designing and building a new stomach guard. In addition, the XBT and MBIT acquisition angle ranges will need to be solidified so that the limit switches can be properly moved such that they prevent unsafe over-rotation, but permit wide-angle acquisition. Once these adjustments have been made to the DMT system, we can begin accruing patients and acquiring data to corroborate our preliminary findings and authenticate the use of the optimized breast immobilization device. 


\section{APPENDIX A}

(Elliptical coordinates of optimized immobilization device)

\begin{tabular}{|c|c|c|c|c|c|c|}
\hline \multirow{2}{*}{$\begin{array}{l}a= \\
b= \\
\theta\left(^{\circ}\right)\end{array}$} & \multirow{2}{*}{$\begin{array}{c}3.385826772 \\
1.732283465 \\
\theta \text { (radians) }\end{array}$} & \multirow{2}{*}{$\begin{array}{l}\text { in } \\
\text { in } \\
\text { r(in) }\end{array}$} & \multicolumn{3}{|c|}{ Green highlight cells represent truncation points } & \multirow[b]{2}{*}{$y(\mathrm{~cm})$} \\
\hline & & & $x$ (in) & $y$ (in) & $x(\mathrm{~cm})$ & \\
\hline 1 & 0.017453293 & 3.3844 & 3.383858023 & 0.059065461 & 8.594999378 & 0.150026272 \\
\hline 2 & 0.034906585 & 3.3800 & 3.377967547 & 0.117961226 & 8.580037569 & 0.299621514 \\
\hline 3 & 0.052359878 & 3.3728 & 3.368202285 & 0.176520002 & 8.555233804 & 0.448360805 \\
\hline 4 & 0.06981317 & 3.3628 & 3.354639259 & 0.234579229 & 8.520783719 & 0.595831241 \\
\hline 5 & 0.087266463 & 3.3501 & 3.337383808 & 0.291983249 & 8.476954871 & 0.741637452 \\
\hline 6 & 0.104719755 & 3.3348 & 3.316567226 & 0.348585262 & 8.424080754 & 0.885406565 \\
\hline 7 & 0.122173048 & 3.3171 & 3.292343921 & 0.404249003 & 8.362553559 & 1.026792467 \\
\hline 8 & 0.13962634 & 3.2970 & 3.264888183 & 0.45885011 & 8.292815985 & 1.165479281 \\
\hline 9 & 0.157079633 & 3.2747 & 3.234390718 & 0.512277164 & 8.215352423 & 1.301183996 \\
\hline 10 & 0.174532925 & 3.2504 & 3.20105505 & 0.564432372 & 8.130679827 & 1.433658225 \\
\hline 11 & 0.191986218 & 3.2243 & 3.165093932 & 0.615231937 & 8.039338587 & 1.56268912 \\
\hline 12 & 0.20943951 & 3.1966 & 3.126725857 & 0.664606097 & 7.941883676 & 1.688099487 \\
\hline 13 & 0.226892803 & 3.1674 & 3.086171772 & 0.712498894 & 7.838876301 & 1.809747192 \\
\hline 14 & 0.244346095 & 3.1368 & 3.043652065 & 0.758867691 & 7.730876244 & 1.927523934 \\
\hline 15 & 0.261799388 & 3.1052 & 2.999383874 & 0.803682487 & 7.61843504 & 2.041353517 \\
\hline 16 & 0.27925268 & 3.0726 & 2.95357876 & 0.846925081 & 7.502090052 & 2.151189706 \\
\hline 17 & 0.296705973 & 3.0392 & 2.906440749 & 0.888588111 & 7.382359503 & 2.257013802 \\
\hline 18 & 0.314159265 & 3.0053 & 2.858164748 & 0.928674022 & 7.259738459 & 2.358832015 \\
\hline 19 & 0.331612558 & 2.9708 & 2.808935324 & 0.967193996 & 7.134695722 & 2.45667275 \\
\hline 20 & 0.34906585 & 2.9360 & 2.758925817 & 1.004166876 & 7.007671576 & 2.550583865 \\
\hline 21 & 0.366519143 & 2.9010 & 2.708297762 & 1.039618107 & 6.879076317 & 2.640629992 \\
\hline 22 & 0.383972435 & 2.8659 & 2.657200578 & 1.073578721 & 6.749289469 & 2.726889951 \\
\hline 23 & 0.401425728 & 2.8308 & 2.605771495 & 1.106084376 & 6.618659598 & 2.809454316 \\
\hline 24 & 0.41887902 & 2.7958 & 2.554135676 & 1.137174469 & 6.487504618 & 2.888423152 \\
\hline 25 & 0.436332313 & 2.7611 & 2.502406504 & 1.166891317 & 6.356112521 & 2.963903945 \\
\hline 26 & 0.453785606 & 2.7266 & 2.450685992 & 1.195279423 & 6.224742421 & 3.036009734 \\
\hline 27 & 0.471238898 & 2.6925 & 2.399065294 & 1.222384822 & 6.093625846 & 3.104857448 \\
\hline 28 & 0.488692191 & 2.6589 & 2.347625281 & 1.248254504 & 5.962968214 & 3.17056644 \\
\hline 29 & 0.506145483 & 2.6256 & 2.296437174 & 1.272935912 & 5.832950422 & 3.233257216 \\
\hline 30 & 0.523598776 & 2.5930 & 2.24556319 & 1.296476512 & 5.703730503 & 3.293050341 \\
\hline 31 & 0.541052068 & 2.5608 & 2.19505721 & 1.318923434 & 5.575445314 & 3.350065523 \\
\hline 32 & 0.558505361 & 2.5293 & 2.144965436 & 1.340323162 & 5.448212208 & 3.404420831 \\
\hline 33 & 0.575958653 & 2.4984 & 2.095327038 & 1.360721289 & 5.322130677 & 3.456232073 \\
\hline 34 & 0.593411946 & 2.4681 & 2.046174779 & 1.380162315 & 5.197283939 & 3.505612281 \\
\hline
\end{tabular}




\begin{tabular}{|c|c|c|c|c|c|c|}
\hline 35 & 0.610865238 & 2.4385 & 1.997535611 & 1.398689492 & 5.073740451 & 3.552671311 \\
\hline 36 & 0.628318531 & 2.4096 & 1.949431237 & 1.416344699 & 4.951555343 & 3.597515537 \\
\hline 37 & 0.645771823 & 2.3814 & 1.901878648 & 1.433168358 & 4.830771765 & 3.640247629 \\
\hline 38 & 0.663225116 & 2.3539 & 1.854890606 & 1.449199369 & 4.711422139 & 3.680966398 \\
\hline 39 & 0.680678408 & 2.3271 & 1.808476113 & 1.46447508 & 4.593529326 & 3.719766704 \\
\hline 40 & 0.698131701 & 2.3010 & 1.762640823 & 1.479031264 & 4.477107689 & 3.756739411 \\
\hline 41 & 0.715584993 & 2.2756 & 1.717387434 & 1.49290212 & 4.362164082 & 3.791971385 \\
\hline 42 & 0.733038286 & 2.2509 & 1.672716039 & 1.506120286 & 4.248698738 & 3.825545527 \\
\hline 43 & 0.750491578 & 2.2269 & 1.628624444 & 1.518716864 & 4.136706089 & 3.857540834 \\
\hline 44 & 0.767944871 & 2.2036 & 1.585108463 & 1.530721449 & 4.026175495 & 3.888032481 \\
\hline 45 & 0.785398163 & 2.1809 & 1.542162172 & 1.542162172 & 3.917091917 & 3.917091917 \\
\hline 46 & 0.802851456 & 2.1590 & 1.499778152 & 1.55306574 & 3.809436506 & 3.944786981 \\
\hline 47 & 0.820304748 & 2.1378 & 1.457947695 & 1.563457489 & 3.703187145 & 3.971182021 \\
\hline 48 & 0.837758041 & 2.1172 & 1.416660992 & 1.573361427 & 3.59831892 & 3.996338025 \\
\hline 49 & 0.855211333 & 2.0972 & 1.375907305 & 1.582800295 & 3.494804555 & 4.02031275 \\
\hline 50 & 0.872664626 & 2.0779 & 1.335675112 & 1.591795613 & 3.392614784 & 4.043160857 \\
\hline 51 & 0.890117919 & 2.0593 & 1.29595224 & 1.600367736 & 3.291718689 & 4.06 \\
\hline 52 & 0.907571211 & 2.0413 & 1.256725982 & 1.608535905 & 3.192083995 & 4.085681198 \\
\hline 53 & 0.925024504 & 2.0238 & 1.217983202 & 1.616318301 & 3.093677333 & 4.105448484 \\
\hline 54 & 0.942477796 & 2.0070 & 1.17971042 & 1.623732093 & 2.996464466 & 4.124279516 \\
\hline 55 & 0.959931089 & 1.9908 & 1.141893894 & 1.630793489 & 2.900410492 & 4.142215463 \\
\hline 56 & 0.977384381 & 1.9752 & 1.104519691 & 1.637517783 & 2.805480016 & 4.159295169 \\
\hline 57 & 0.994837674 & 1.9601 & 1.067573741 & 1.6439194 & 2.711637303 & 4.175555277 \\
\hline 58 & 1.012290966 & 1.9457 & 1.031041892 & 1.650011941 & 2.618846407 & 4.191030331 \\
\hline 59 & 1.029744259 & 1.9317 & 0.994909955 & 1.655808225 & 2.527071285 & 4.20575289 \\
\hline 60 & 1.047197551 & 1.9183 & 0.959163737 & 1.661320326 & 2.436275893 & 4.219753627 \\
\hline 61 & 1.064650844 & 1.9055 & 0.92378908 & 1.666559616 & 2.346424263 & 4.233061425 \\
\hline 62 & 1.082104136 & 1.8931 & 0.888771881 & 1.671536799 & 2.257480579 & 4.24570347 \\
\hline 63 & 1.099557429 & 1.8813 & 0.85409812 & 1.676261943 & 2.169409225 & 4.257705336 \\
\hline 64 & 1.117010721 & 1.8700 & 0.819753873 & 1.680744516 & 2.082174838 & 4.26909107 \\
\hline 65 & 1.134464014 & 1.8592 & 0.785725332 & 1.684993412 & 1.995742344 & 4.279883268 \\
\hline 66 & 1.151917306 & 1.8489 & 0.751998812 & 1.689016986 & 1.910076983 & 4.290103146 \\
\hline 67 & 1.169370599 & 1.8390 & 0.718560764 & 1.692823075 & 1.82514434 & 4.299770611 \\
\hline 68 & 1.186823891 & 1.8296 & 0.685397777 & 1.696419027 & 1.740910353 & 4.308904327 \\
\hline 69 & 1.204277184 & 1.8207 & 0.652496586 & 1.699811721 & 1.657341328 & 4.31752177 \\
\hline 70 & 1.221730476 & 1.8123 & 0.619844073 & 1.703007594 & 1.574403945 & 4.325639289 \\
\hline 71 & 1.239183769 & 1.8043 & 0.587427267 & 1.70601266 & 1.492065259 & 4.333272156 \\
\hline 72 & 1.256637061 & 1.7968 & 0.555233345 & 1.708832527 & 1.410292697 & 4.340434617 \\
\hline 73 & 1.274090354 & 1.7897 & 0.523249628 & 1.711472417 & 1.329054056 & 4.347139938 \\
\hline 74 & 1.291543646 & 1.7830 & 0.491463579 & 1.713937183 & 1.24831749 & 4.353400446 \\
\hline 75 & 1.308996939 & 1.7768 & 0.459862798 & 1.716231326 & 1.168051506 & 4.359227567 \\
\hline
\end{tabular}




\begin{tabular}{|c|c|c|c|c|c|c|}
\hline 76 & 1.326450232 & 1.7710 & 0.428435018 & 1.718359003 & 1.088224947 & 4.364631868 \\
\hline 77 & 1.343903524 & 1.7656 & 0.397168101 & 1.720324049 & 1.008806977 & 4.369623084 \\
\hline 78 & 1.361356817 & 1.7606 & 0.366050028 & 1.722129982 & 0.92976707 & 4.374210155 \\
\hline 79 & 1.378810109 & 1.7560 & 0.335068893 & 1.723780019 & 0.851074988 & 4.378401247 \\
\hline 80 & 1.396263402 & 1.7519 & 0.304212899 & 1.725277081 & 0.772700762 & 4.382203786 \\
\hline 81 & 1.413716694 & 1.7481 & 0.273470346 & 1.726623809 & 0.694614678 & 4.385624475 \\
\hline 82 & 1.431169987 & 1.7448 & 0.242829626 & 1.727822566 & 0.616787249 & 4.388669317 \\
\hline 83 & 1.448623279 & 1.7419 & 0.212279213 & 1.728875447 & 0.5391892 & 4.391343635 \\
\hline 84 & 1.466076572 & 1.7393 & 0.181807655 & 1.729784286 & 0.461791443 & 4.393652087 \\
\hline 85 & 1.483529864 & 1.7372 & 0.151403565 & 1.730550662 & 0.384565054 & 4.395598683 \\
\hline 86 & 1.500983157 & 1.7354 & 0.121055612 & 1.731175902 & 0.307481254 & 4.397186791 \\
\hline 87 & 1.518436449 & 1.7340 & 0.090752512 & 1.731661086 & 0.23051138 & 4.398419158 \\
\hline 88 & 1.535889742 & 1.7331 & 0.060483019 & 1.73200705 & 0.153626868 & 4.399297907 \\
\hline 89 & 1.553343034 & 1.7325 & 0.030235915 & 1.732214391 & 0.076799223 & 4.399824552 \\
\hline 90 & 1.570796327 & 1.7323 & $1.06115 \mathrm{E}-16$ & 1.732283465 & $2.69533 \mathrm{E}-16$ & 4.4 \\
\hline 91 & 1.588249619 & 1.7325 & -0.030235915 & 1.732214391 & -0.076799223 & 4.399824552 \\
\hline 92 & 1.605702912 & 1.7331 & -0.060483019 & 1.73200705 & -0.153626868 & 7907 \\
\hline 93 & 1.623156204 & 1.7340 & -0.090752512 & 1.731661086 & -0.23051138 & 4.398419158 \\
\hline 94 & 1.640609497 & 1.7354 & -0.121055612 & 1.731175902 & -0.307481254 & 4.397186791 \\
\hline 95 & 1.658062789 & 1.7372 & -0.151403565 & 1.730550662 & -0.384565054 & 4.395598683 \\
\hline 96 & 1.675516082 & 1.7393 & -0.181807655 & 1.729784286 & -0.461791443 & 4.393652087 \\
\hline 97 & 1.692969374 & 1.7419 & -0.212279213 & 1.728875447 & -0.5391892 & 4.391343635 \\
\hline 98 & 1.710422667 & 1.7448 & -0.242829626 & 1.727822566 & -0.616787249 & 4.388669317 \\
\hline 99 & 1.727875959 & 1.7481 & -0.273470346 & 1.726623809 & -0.694614678 & 4.385624475 \\
\hline 100 & 1.745329252 & 1.7519 & -0.304212899 & 7081 & -0.772700762 & 03786 \\
\hline 101 & 1.762782545 & 1.7560 & -0.335068893 & 1.723780019 & -0.851074988 & 4.378401247 \\
\hline 102 & 1.780235837 & 1.7606 & -0.366050028 & 1.722129982 & -0.92976707 & 4.374210155 \\
\hline 103 & 1.79768913 & 1.7656 & -0.397168101 & 1.720324049 & -1.008806977 & 4.369623084 \\
\hline 104 & 1.815142422 & 1.7710 & -0.428435018 & 1.718359003 & -1.088224947 & 4.364631868 \\
\hline 105 & 1.832595715 & 1.7768 & -0.459862798 & 1.716231326 & -1.168051506 & 4.359227567 \\
\hline 106 & 1.850049007 & 1.7830 & -0.491463579 & 1.713937183 & -1.24831749 & 4.353400446 \\
\hline 107 & 1.8675023 & 1.7897 & -0.523249628 & 1.711472417 & -1.329054056 & 4.347139938 \\
\hline 108 & 1.884955592 & 1.7968 & -0.555233345 & 1.708832527 & -1.410292697 & 4.340434617 \\
\hline 109 & 1.902408885 & 1.8043 & -0.587427267 & 1.70601266 & -1.492065259 & 4.333272156 \\
\hline 110 & 1.919862177 & 1.8123 & -0.619844073 & 1.703007594 & -1.574403945 & 4.325639289 \\
\hline 111 & 1.93731547 & 1.8207 & -0.652496586 & 1.699811721 & -1.657341328 & 4.31752177 \\
\hline 112 & 1.954768762 & 1.8296 & -0.685397777 & 1.696419027 & -1.740910353 & 4.308904327 \\
\hline 113 & 1.972222055 & 1.8390 & -0.718560764 & 1.692823075 & -1.82514434 & 4.299770611 \\
\hline 114 & 1.989675347 & 1.8489 & -0.751998812 & 1.689016986 & -1.910076983 & 4.290103146 \\
\hline 115 & 2.00712864 & 1.8592 & -0.785725332 & 1.684993412 & -1.995742344 & 4.279883268 \\
\hline 116 & 2.024581932 & 1.8700 & -0.819753873 & 1.680744516 & -2.082174838 & 4.26909107 \\
\hline
\end{tabular}




\begin{tabular}{|c|c|c|c|c|c|c|}
\hline 117 & 2.042035225 & 1.8813 & -0.85409812 & 1.676261943 & -2.169409225 & 4.257705336 \\
\hline 118 & 2.059488517 & 1.8931 & -0.888771881 & 1.671536799 & -2.257480579 & 4.24570347 \\
\hline 119 & 2.07694181 & 9055 & -0.92378908 & 1.666559616 & -2.346424263 & 061425 \\
\hline 120 & 2.094395102 & 1.9183 & -0.959163737 & 1.661320326 & -2.436275893 & 4.219753627 \\
\hline 121 & 2.111848395 & 1.9317 & -0.994909955 & 1.655808225 & -2.527071285 & 4.20575289 \\
\hline 122 & 2.129301687 & 1.9457 & -1.031041892 & 1.650011941 & -2.618846407 & 4.191030331 \\
\hline 123 & 2.14675498 & 1.9601 & -1.067573741 & 1.6439194 & -2.711637303 & 4.175555277 \\
\hline 124 & 2.164208272 & 1.9752 & -1.104519691 & 1.637517783 & -2.805480016 & 4.159295169 \\
\hline 125 & 2.181661565 & 1.9908 & -1.141893894 & 1.630793489 & -2.900410492 & 4.142215463 \\
\hline 126 & 2.199114858 & 2.0070 & -1.17971042 & 1.623732093 & -2.996464466 & 4.124279516 \\
\hline 127 & 2.21656815 & 2.0238 & -1.217983202 & 1.616318301 & -3.093677333 & 4.105448484 \\
\hline 128 & 2.234021443 & 2.0413 & -1.256725982 & 1.608535905 & -3.192083995 & 4.085681198 \\
\hline 129 & 2.251474735 & 2.0593 & -1.29595224 & 1.600367736 & -3.291718689 & 4.064934049 \\
\hline 130 & 2.268928028 & 2.0779 & -1.335675112 & 1795613 & -3.392614784 & 60857 \\
\hline 131 & 2.28638132 & 2.0972 & -1.375907305 & 1.582800295 & -3.494804555 & 4.02031275 \\
\hline 132 & 2.303834613 & 2.1172 & -1.416660992 & 1.573361427 & -3.59831892 & 3.996338025 \\
\hline 133 & 2.321287905 & 2.1378 & -1.457947695 & 1.563457489 & -3.703187145 & 3.971182021 \\
\hline 134 & 2.338741198 & 2.1590 & -1.499778152 & 1.55306574 & -3.809436506 & 3.944786981 \\
\hline 135 & 2.35619449 & 2.1809 & -1.542162172 & 1.542162172 & -3.917091917 & 3.917091917 \\
\hline 136 & 2.373647783 & 2.2036 & -1.585108463 & 1.530721449 & -4.026175495 & 3.888032481 \\
\hline 137 & 2.391101075 & 2.2269 & -1.628624444 & 1.518716864 & -4.136706089 & 3.857540834 \\
\hline 138 & 2.408554368 & 2.2509 & -1.672716039 & 1.506120286 & -4.248698738 & 3.825545527 \\
\hline 139 & 2.42600766 & 2.2756 & -1.717387434 & 1.49290212 & -4.362164082 & 3.791971385 \\
\hline 140 & 2.443460953 & 2.3010 & -1.762640823 & 1.479031264 & -4.477107689 & 3.756739411 \\
\hline 141 & 2.460914245 & 2.3271 & -1.808476113 & 1.46447508 & -4.593529326 & 3.719766704 \\
\hline 142 & 2.478367538 & 2.3539 & -1.854890606 & 1.449199369 & -4.711422139 & 3.680966398 \\
\hline 143 & 2.49582083 & 2.3814 & -1.901878648 & 1.433168358 & -4.830771765 & 3.640247629 \\
\hline 144 & 2.513274123 & 2.4096 & -1.949431237 & 1.416344699 & -4.951555343 & 3.597515537 \\
\hline 145 & 2.530727415 & 2.4385 & -1.997535611 & 1.398689492 & -5.073740451 & 3.552671311 \\
\hline 146 & 2.548180708 & 2.4681 & -2.046174779 & 1.380162315 & -5.197283939 & 3.505612281 \\
\hline 147 & 2.565634 & 2.4984 & -2.095327038 & 1.360721289 & -5.322130677 & 3.456232073 \\
\hline 148 & 2.583087293 & 2.5293 & -2.144965436 & 1.340323162 & -5.448212208 & 3.404420831 \\
\hline 149 & 2.600540585 & 2.5608 & -2.19505721 & 1.318923434 & -5.575445314 & 3.350065523 \\
\hline 150 & 2.617993878 & 2.5930 & -2.24556319 & 1.296476512 & -5.703730503 & 3.293050341 \\
\hline 151 & 2.635447171 & 2.6256 & -2.296437174 & 1.272935912 & -5.832950422 & 3.233257216 \\
\hline 152 & 2.652900463 & 2.6589 & -2.347625281 & 1.248254504 & -5.962968214 & 3.17056644 \\
\hline 153 & 2.670353756 & 2.6925 & -2.399065294 & 1.222384822 & -6.093625846 & 3.104857448 \\
\hline 154 & 2.687807048 & 2.7266 & -2.450685992 & 1.195279423 & -6.224742421 & 3.036009734 \\
\hline 155 & 2.705260341 & 2.7611 & -2.502406504 & 1.166891317 & -6.356112521 & 2.963903945 \\
\hline 156 & 2.722713633 & 2.7958 & -2.554135676 & 1.137174469 & -6.487504618 & 2.888423152 \\
\hline 157 & 2.740166926 & 2.8308 & -2.605771495 & 1.106084376 & -6.618659598 & 2.809454316 \\
\hline
\end{tabular}




\begin{tabular}{|c|c|c|c|c|c|c|}
\hline 158 & 2.757620218 & 2.8659 & -2.657200578 & 1.073578721 & -6.749289469 & 2.726889951 \\
\hline 159 & 2.775073511 & 2.9010 & -2.708297762 & 1.039618107 & -6.879076317 & 2.640629992 \\
\hline 160 & 2.792526803 & 2.9360 & -2.758925817 & 1.004166876 & -7.007671576 & 2.550583865 \\
\hline 161 & 2.809980096 & 2.9708 & -2.808935324 & 0.967193996 & -7.134695722 & 2.45667275 \\
\hline 162 & 2.827433388 & 3.0053 & -2.858164748 & 0.928674022 & -7.259738459 & 2.358832015 \\
\hline 163 & 2.844886681 & 3.0392 & -2.906440749 & 0.888588111 & -7.382359503 & 2.257013802 \\
\hline 164 & 2.862339973 & 3.0726 & -2.95357876 & 0.846925081 & -7.502090052 & 2.151189706 \\
\hline 165 & 2.879793266 & 3.1052 & -2.999383874 & 0.803682487 & -7.61843504 & 2.041353517 \\
\hline 166 & 2.897246558 & 3.1368 & -3.043652065 & 0.758867691 & -7.730876244 & 1.927523934 \\
\hline 167 & 2.914699851 & 3.1674 & -3.086171772 & 0.712498894 & -7.838876301 & 1.809747192 \\
\hline 168 & 2.932153143 & 3.1966 & -3.126725857 & 0.664606097 & -7.941883676 & 1.688099487 \\
\hline 169 & 2.949606436 & 3.2243 & -3.165093932 & 0.615231937 & -8.039338587 & 1.56268912 \\
\hline 170 & 2.967059728 & 3.2504 & -3.20105505 & 0.564432372 & -8.130679827 & 1.433658225 \\
\hline 171 & 2.984513021 & 3.2747 & -3.234390718 & 277164 & -8.215352423 & 996 \\
\hline 172 & 3.001966313 & 3.2970 & -3.264888183 & 0.45885011 & -8.292815985 & 281 \\
\hline 173 & 3.019419606 & 3.3171 & -3.25 & 0.404249003 & -8.362553559 & 467 \\
\hline 174 & 3.036872898 & 3.3348 & -3.316 & 262 & -8.424080754 & 565 \\
\hline 175 & 3.054326191 & 3.3501 & -3.337383808 & 0.291983249 & -8.476954871 & 0.741637452 \\
\hline 176 & 3.071779484 & 3.3628 & -3.354639259 & 0.234579229 & -8.520783719 & 0.595831241 \\
\hline 177 & 3.089232776 & 3.3728 & -3.368202285 & 0.176520002 & -8.555233804 & 0.448360805 \\
\hline 178 & 3.106686069 & 3.3800 & -3.377967547 & 0.117961226 & -8.580037569 & 0.299621514 \\
\hline 179 & 3.124139361 & 3.3844 & -3.383858023 & 0.059065461 & -8.594999378 & 0.150026272 \\
\hline 180 & 3.141592654 & 3.3858 & -3.385826772 & $4.14814 \mathrm{E}-16$ & -8.6 & $1.05363 \mathrm{E}-15$ \\
\hline 181 & 3.159045946 & 3.3844 & -3.383858023 & -0.059065461 & -8.594999378 & -0.150026272 \\
\hline 182 & 3.176499239 & 3.3800 & -3.377967547 & -0.117961226 & -8.580037569 & 621514 \\
\hline 183 & 52531 & 3.3728 & -3.36 & 0002 & 233804 & 805 \\
\hline 184 & 3.211405824 & 3.3628 & -3.354639259 & -0.234579229 & -8.520783719 & -0.595831241 \\
\hline 185 & 3.228859116 & 3.3501 & -3.337383808 & -0.291983249 & -8.476954871 & -0.741637452 \\
\hline 186 & 3.246312409 & 3.3348 & -3.316567226 & -0.348585262 & -8.424080754 & -0.885406565 \\
\hline 187 & 3.263765701 & 3.3171 & -3.292343921 & -0.404249003 & -8.362553559 & -1.026792467 \\
\hline 188 & 3.281218994 & 3.2970 & -3.264888183 & -0.45885011 & -8.292815985 & -1.165479281 \\
\hline 189 & 3.298672286 & 3.2747 & -3.234390718 & -0.512277164 & -8.215352423 & -1.301183996 \\
\hline 190 & 3.316125579 & 3.2504 & -3.20105505 & -0.564432372 & -8.130679827 & -1.433658225 \\
\hline 191 & 3.333578871 & 3.2243 & -3.165093932 & -0.615231937 & -8.039338587 & -1.56268912 \\
\hline 192 & 3.351032164 & 3.1966 & -3.126725857 & -0.664606097 & -7.941883676 & -1.688099487 \\
\hline 193 & 3.368485456 & 3.1674 & -3.086171772 & -0.712498894 & -7.838876301 & -1.809747192 \\
\hline 194 & 3.385938749 & 3.1368 & -3.043652065 & -0.758867691 & -7.730876244 & -1.927523934 \\
\hline 195 & 3.403392041 & 3.1052 & -2.999383874 & -0.803682487 & -7.61843504 & -2.041353517 \\
\hline 196 & 3.420845334 & 3.0726 & -2.95357876 & -0.846925081 & -7.502090052 & -2.151189706 \\
\hline 197 & 3.438298626 & 3.0392 & -2.906440749 & -0.888588111 & -7.382359503 & -2.257013802 \\
\hline 198 & 3.455751919 & 3.0053 & -2.858164748 & -0.928674022 & -7.259738459 & -2.358832015 \\
\hline
\end{tabular}




\begin{tabular}{|c|c|c|c|c|c|c|}
\hline 199 & 3.473205211 & 2.9708 & -2.808935324 & -0.967193996 & -7.134695722 & -2.45667275 \\
\hline 200 & 3.490658504 & 2.9360 & -2.758925817 & -1.004166876 & -7.007671576 & -2.550583865 \\
\hline 201 & 08111797 & 9010 & -2.708297762 & -1.039618107 & -6.879076317 & -2.6406 \\
\hline 202 & 3.525565089 & 2.8659 & -2.657200578 & -1.073578721 & -6.749289469 & -2.726889951 \\
\hline 203 & 3.543018382 & 2.8308 & -2.605771495 & -1.106084376 & -6.618659598 & -2.809454316 \\
\hline 204 & 3.560471674 & 2.7958 & -2.554135676 & -1.137174469 & -6.487504618 & -2.888 \\
\hline 205 & 3.577924967 & 2.7611 & -2.502406504 & -1.166891317 & -6.356112521 & -2.963903945 \\
\hline 206 & 3.595378259 & 2.7266 & -2.450685992 & -1.195279423 & -6.224742421 & -3.036009734 \\
\hline 207 & 3.612831552 & 2.6925 & -2.399065294 & -1.222384822 & -6.093625846 & -3.104857448 \\
\hline 208 & 3.630284844 & 2.6589 & -2.347625281 & -1.248254504 & -5.962968214 & -3.17056644 \\
\hline 209 & 3.647738137 & 2.6256 & -2.296437174 & -1.272935912 & -5.832950422 & -3.233257216 \\
\hline 210 & 3.665191429 & 2.5930 & -2.24556319 & -1.296476512 & -5.703730503 & -3.293050341 \\
\hline 211 & 3.682644722 & 2.5608 & -2.19505721 & -1.318923434 & -5.575445314 & -3.3500 \\
\hline 212 & 3.700098014 & 2.5 & -2.14 & -1.340323162 & -5.448212208 & -3.404 \\
\hline 213 & 3.717551307 & 2.4984 & -2.095327038 & -1.360721289 & -5.3221 & -3.456 \\
\hline 214 & 3.735004599 & 2.4681 & -2.04617 & -1.38016 & -5.197283939 & -3.505 \\
\hline 215 & 3.752457892 & 2.4385 & -1.99753 & -1.398 & -5.0737 & -3.55 \\
\hline 216 & 3.769911184 & 2.4096 & -1.949431237 & -1.416344699 & -4.951555343 & -3.597515537 \\
\hline 217 & 3.787364477 & 2.3814 & -1.901878648 & -1.433168358 & -4.830771765 & -3.640247629 \\
\hline 218 & 3.804817769 & 2.3539 & -1.854890606 & -1.449199369 & -4.711422139 & -3.680966398 \\
\hline 219 & 3.822271062 & 2.3271 & -1.808476113 & -1.46447508 & -4.593529326 & -3.719766704 \\
\hline 220 & 3.839724354 & 2.3010 & -1.762640823 & -1.479031264 & -4.477107689 & -3.756739411 \\
\hline 221 & 3.857177647 & 2.2756 & -1.717387434 & -1.49290212 & -4.362164082 & -3.791971385 \\
\hline 222 & 3.874630939 & 2.2509 & -1.672716039 & -1.506120286 & -4.248698738 & -3.825 \\
\hline 223 & 3.892084232 & 2.2269 & -1.628624444 & -1.518716864 & -4.136706089 & -3.8575 \\
\hline 224 & 3.909537524 & 2.2036 & -1.585108463 & -1.530721449 & -4.026175495 & -3.888032481 \\
\hline 225 & 3.926990817 & 2.1809 & -1.542162172 & -1.542162172 & -3.917091917 & -3.917091917 \\
\hline 226 & 3.94444411 & 2.1590 & -1.499778152 & -1.55306574 & -3.809436506 & -3.944786981 \\
\hline 227 & 3.961897402 & 2.1378 & -1.457947695 & -1.563457489 & -3.703187145 & -3.971182021 \\
\hline 228 & 3.979350695 & 2.1172 & -1.416660992 & -1.573361427 & -3.59831892 & -3.996338025 \\
\hline 229 & 3.996803987 & 2.0972 & -1.375907305 & -1.582800295 & -3.494804555 & -4.02031275 \\
\hline 230 & 4.01425728 & 2.0779 & -1.335675112 & -1.591795613 & -3.392614784 & -4.043160857 \\
\hline 231 & 4.031710572 & 2.0593 & -1.29595224 & -1.600367736 & -3.291718689 & -4.064934049 \\
\hline 232 & 4.049163865 & 2.0413 & -1.256725982 & -1.608535905 & -3.192083995 & -4.085681198 \\
\hline 233 & 4.066617157 & 2.0238 & -1.217983202 & -1.616318301 & -3.093677333 & -4.105448484 \\
\hline 234 & 4.08407045 & 2.0070 & -1.17971042 & -1.623732093 & -2.996464466 & -4.124279516 \\
\hline 235 & 4.101523742 & 1.9908 & -1.141893894 & -1.630793489 & -2.900410492 & -4.142215463 \\
\hline 236 & 4.118977035 & 1.9752 & -1.104519691 & -1.637517783 & -2.805480016 & -4.159295169 \\
\hline 237 & 4.136430327 & 1.9601 & -1.067573741 & -1.6439194 & -2.711637303 & -4.175555277 \\
\hline 238 & 4.15388362 & 1.9457 & -1.031041892 & -1.650011941 & -2.618846407 & -4.191030331 \\
\hline 239 & 4.171336912 & 1.9317 & -0.994909955 & -1.655808225 & -2.527071285 & -4.20575289 \\
\hline
\end{tabular}




\begin{tabular}{|c|c|c|c|c|c|c|}
\hline 240 & 4.188790205 & 1.9183 & -0.959163737 & -1.661320326 & -2.436275893 & -4.219753627 \\
\hline 241 & 4.206243497 & 1.9055 & -0.92378908 & -1.666559616 & -2.346424263 & -4.233061425 \\
\hline 242 & 4.22369679 & 931 & -0.888771881 & -1.671536799 & -2.257480579 & -4.24570347 \\
\hline 243 & 4.241150082 & 1.8813 & -0.85409812 & -1.676261943 & -2.169409225 & -4.257705336 \\
\hline 244 & 4.258603375 & 1.8700 & -0.819753873 & -1.680744516 & -2.082174838 & -4.26909107 \\
\hline 245 & 4.276056667 & 1.8592 & -0.785725332 & -1.684993412 & -1.995742344 & -4.279883268 \\
\hline 246 & 4.29350996 & 1.8489 & -0.751998812 & -1.689016986 & -1.910076983 & -4.290103146 \\
\hline 247 & 4.310963252 & 1.8390 & -0.718560764 & -1.692823075 & -1.82514434 & -4.299770611 \\
\hline 248 & 4.328416545 & 1.8296 & -0.685397777 & -1.696419027 & -1.740910353 & -4.308904327 \\
\hline 249 & 4.345869837 & 1.8207 & -0.652496586 & -1.699811721 & -1.657341328 & -4.31752177 \\
\hline 250 & 4.36332313 & 1.8123 & -0.619844073 & -1.703007594 & -1.574403945 & -4.325639289 \\
\hline 251 & 4.380776423 & 1.8043 & -0.587427267 & -1.70601266 & -1.492065259 & -4.333272156 \\
\hline 252 & 4.398229715 & 1.7968 & -0.555233345 & -1.708832527 & -1.410292697 & -4.340434617 \\
\hline 253 & 4.415683008 & 1.7897 & -0.523249628 & -1.711472417 & -1.329054056 & -4.347139938 \\
\hline 254 & 4.4331363 & 1.7830 & -0.491463579 & -1.713937183 & -1.24831749 & 446 \\
\hline 255 & 4.450589593 & 1.7768 & -0.45986 & -1.71623 & -1.168051506 & -4.359 \\
\hline 256 & 4.468042885 & 1.7710 & -0.428435018 & -1.7183 & -1.088224947 & -4.36 \\
\hline 257 & 4.485496178 & 1.7656 & -0.397168101 & -1.720324049 & -1.008806977 & -4.369623084 \\
\hline 258 & 4.50294947 & 1.7606 & -0.366050028 & -1.722129982 & -0.92976707 & -4.374210155 \\
\hline 259 & 4.520402763 & 1.7560 & -0.335068893 & -1.723780019 & -0.851074988 & -4.378401247 \\
\hline 260 & 4.537856055 & 1.7519 & -0.304212899 & -1.725277081 & -0.772700762 & -4.382203786 \\
\hline 261 & 4.555309348 & 1.7481 & -0.273470346 & -1.726623809 & -0.694614678 & -4.385624475 \\
\hline 262 & 4.57276264 & 1.7448 & -0.242829626 & -1.727822566 & -0.616787249 & -4.388669317 \\
\hline 263 & 4.5902 & 1.7419 & -0.212279213 & -1.728875447 & 1892 & 43635 \\
\hline 264 & 4.607669225 & 1.7393 & -0.181807655 & -1.729784286 & -0.461791443 & -4.393 \\
\hline 265 & 4.625122518 & 1.7372 & -0.151403565 & -1.730550662 & -0.384565054 & -4.395598683 \\
\hline 266 & 4.64257581 & 1.7354 & -0.121055612 & -1.731175902 & -0.307481254 & -4.397186791 \\
\hline 267 & 4.660029103 & 1.7340 & -0.090752512 & -1.731661086 & -0.23051138 & -4.398419158 \\
\hline 268 & 4.677482395 & 1.7331 & -0.060483019 & -1.73200705 & -0.153626868 & -4.399297907 \\
\hline 269 & 4.694935688 & 1.7325 & -0.030235915 & -1.732214391 & -0.076799223 & -4.399824552 \\
\hline 270 & 4.71238898 & 1.7323 & $-3.18346 \mathrm{E}-16$ & -1.732283465 & $-8.08598 \mathrm{E}-16$ & -4.4 \\
\hline 271 & 4.729842273 & 1.7325 & 0.030235915 & -1.732214391 & 0.076799223 & -4.399824552 \\
\hline 272 & 4.747295565 & 1.7331 & 0.060483019 & -1.73200705 & 0.153626868 & -4.399297907 \\
\hline 273 & 4.764748858 & 1.7340 & 0.090752512 & -1.731661086 & 0.23051138 & -4.398419158 \\
\hline 274 & 4.78220215 & 1.7354 & 0.121055612 & -1.731175902 & 0.307481254 & -4.397186791 \\
\hline 275 & 4.799655443 & 1.7372 & 0.151403565 & -1.730550662 & 0.384565054 & -4.395598683 \\
\hline 276 & 4.817108736 & 1.7393 & 0.181807655 & -1.729784286 & 0.461791443 & -4.393652087 \\
\hline 277 & 4.834562028 & 1.7419 & 0.212279213 & -1.728875447 & 0.5391892 & -4.391343635 \\
\hline 278 & 4.852015321 & 1.7448 & 0.242829626 & -1.727822566 & 0.616787249 & -4.388669317 \\
\hline 279 & 4.869468613 & 1.7481 & 0.273470346 & -1.726623809 & 0.694614678 & -4.385624475 \\
\hline 280 & 4.886921906 & 1.7519 & 0.304212899 & -1.725277081 & 0.772700762 & -4.382203786 \\
\hline
\end{tabular}




\begin{tabular}{|c|c|c|c|c|c|c|}
\hline 281 & 4.904375198 & 1.7560 & 0.335068893 & -1.723780019 & 0.851074988 & -4.378401247 \\
\hline 282 & 4.921828491 & 1.7606 & 0.366050028 & -1.722129982 & 0.92976707 & -4.374210155 \\
\hline 283 & 4.939281783 & 1.7656 & 0.397168101 & -1.720324049 & 1.008806977 & -4.369623084 \\
\hline 284 & 4.956735076 & 1.7710 & 0.428435018 & -1.718359003 & 1.088224947 & -4.364631868 \\
\hline 285 & 4.974188368 & 1.7768 & 0.459862798 & -1.716231326 & 1.168051506 & -4.359227567 \\
\hline 286 & 4.991641661 & 1.7830 & 0.491463579 & -1.713937183 & 1.24831749 & -4.353400446 \\
\hline 287 & 5.009094953 & 1.7897 & 0.523249628 & -1.711472417 & 1.329054056 & -4.347139938 \\
\hline 288 & 5.026548246 & 1.7968 & 0.555233345 & -1.708832527 & 1.410292697 & -4.340434617 \\
\hline 289 & 5.044001538 & 1.8043 & 0.587427267 & -1.70601266 & 1.492065259 & -4.333272156 \\
\hline 290 & 5.061454831 & 1.8123 & 0.619844073 & -1.703007594 & 1.574403945 & -4.325639289 \\
\hline 291 & 5.078908123 & 1.8207 & 0.652496586 & -1.699811721 & 1.657341328 & -4.31752177 \\
\hline 292 & 5.096361416 & 1.8296 & 0.685397777 & -1.696419027 & 1.740910353 & -4.308904327 \\
\hline 293 & 5.113814708 & 1.8390 & 0.718560764 & -1.692823075 & 1.82514434 & -4.299770611 \\
\hline 294 & 5.131268001 & 1.8489 & 0.751998812 & -1.689016986 & 1.910076983 & -4.290103146 \\
\hline 295 & 5.148721293 & 1.8592 & 0.785725332 & -1.684993412 & 1.995742344 & -4.279883268 \\
\hline 296 & 5.166174586 & 1.8700 & 0.819753873 & -1.680744516 & 2.082174838 & -4.26909107 \\
\hline 297 & 5.183627878 & 1.8813 & 0.85409812 & -1.676261943 & 2.169409225 & -4.257705336 \\
\hline 298 & 5.201081171 & 1.8931 & 0.888771881 & -1.671536799 & 2.257480579 & -4.24570347 \\
\hline 299 & 5.218534463 & 1.9055 & 0.92378908 & -1.666559616 & 2.346424263 & -4.233061425 \\
\hline 300 & 5.235987756 & 1.9183 & 0.959163737 & -1.661320326 & 2.436275893 & -4.219753627 \\
\hline 301 & 5.253441049 & 1.9317 & 0.994909955 & -1.655808225 & 2.527071285 & -4.20575289 \\
\hline 302 & 5.270894341 & 1.9457 & 1.031041892 & -1.650011941 & 2.618846407 & -4.191030331 \\
\hline 303 & 5.288347634 & 1.9601 & 1.067573741 & -1.6439194 & 2.711637303 & -4.175555277 \\
\hline 304 & 5.305800926 & 1.9752 & 1.104519691 & -1.637517783 & 2.805480016 & -4.159295169 \\
\hline 305 & 5.323254219 & 1.9908 & 1.141893894 & -1.630793489 & 2.900410492 & 2215463 \\
\hline 306 & 5.340707511 & 2.0070 & 971042 & -1.623732093 & 2.996464466 & -4.124279516 \\
\hline 307 & 5.358160804 & 2.0238 & 1.217983202 & -1.616318301 & 3.093677333 & -4.105448484 \\
\hline 308 & 5.375614096 & 2.0413 & 1.256725982 & -1.608535905 & 3.192083995 & -4.085681198 \\
\hline 309 & 5.393067389 & 2.0593 & 1.29595224 & -1.600367736 & 3.291718689 & -4.064934049 \\
\hline 310 & 5.410520681 & 2.0779 & 1.335675112 & -1.591795613 & 3.392614784 & -4.043160857 \\
\hline 311 & 5.427973974 & 2.0972 & 1.375907305 & -1.582800295 & 3.494804555 & -4.02031275 \\
\hline 312 & 5.445427266 & 2.1172 & 1.416660992 & -1.573361427 & 3.59831892 & -3.996338025 \\
\hline 313 & 5.462880559 & 2.1378 & 1.457947695 & -1.563457489 & 3.703187145 & -3.971182021 \\
\hline 314 & 5.480333851 & 2.1590 & 1.499778152 & -1.55306574 & 3.809436506 & -3.944786981 \\
\hline 315 & 5.497787144 & 2.1809 & 1.542162172 & -1.542162172 & 3.917091917 & -3.917091917 \\
\hline 316 & 5.515240436 & 2.2036 & 1.585108463 & -1.530721449 & 4.026175495 & -3.888032481 \\
\hline 317 & 5.532693729 & 2.2269 & 1.628624444 & -1.518716864 & 4.136706089 & -3.857540834 \\
\hline 318 & 5.550147021 & 2.2509 & 1.672716039 & -1.506120286 & 4.248698738 & -3.825545527 \\
\hline 319 & 5.567600314 & 2.2756 & 1.717387434 & -1.49290212 & 4.362164082 & -3.791971385 \\
\hline 320 & 5.585053606 & 2.3010 & 1.762640823 & -1.479031264 & 4.477107689 & -3.756739411 \\
\hline 321 & 5.602506899 & 2.3271 & 1.808476113 & -1.46447508 & 4.593529326 & -3.719766704 \\
\hline
\end{tabular}




\begin{tabular}{|c|c|c|c|c|c|c|}
\hline 322 & 5.619960191 & 2.3539 & 1.854890606 & -1.449199369 & 4.711422139 & -3.680966398 \\
\hline 323 & 5.637413484 & 2.3814 & 1.901878648 & -1.433168358 & 4.830771765 & -3.640247629 \\
\hline 324 & 5.654866776 & 2.4096 & 1.949431237 & -1.416344699 & 4.951555343 & -3.597515537 \\
\hline 325 & 5.672320069 & 2.4385 & 1.997535611 & -1.398689492 & 5.073740451 & -3.552671311 \\
\hline 326 & 5.689773362 & 2.4681 & 2.046174779 & -1.380162315 & 5.197283939 & -3.505612281 \\
\hline 327 & 5.707226654 & 2.4984 & 2.095327038 & -1.360721289 & 5.322130677 & -3.456232073 \\
\hline 328 & 5.724679947 & 2.5293 & 2.144965436 & -1.340323162 & 5.448212208 & -3.404420831 \\
\hline 329 & 5.742133239 & 2.5608 & 2.19505721 & -1.318923434 & 5.575445314 & -3.3500 \\
\hline 330 & 5.759586532 & 2.5930 & 5319 & -1.296476512 & 5.703730503 & -3.2930 \\
\hline 331 & 5.777039824 & 2.6256 & 2.296437174 & -1.272935912 & 5.832950422 & -3.233257216 \\
\hline 332 & 5.794493117 & 2.6589 & 2.347625281 & -1.248254504 & 5.962968214 & -3.17056644 \\
\hline 333 & 5.811946409 & 2.6925 & 2.399065294 & -1.222384822 & 6.093625846 & -3.104857448 \\
\hline 334 & 5.829399702 & 2.7266 & 2.450685992 & -1.195279423 & 6.224742421 & -3.036009734 \\
\hline 335 & 5.846852994 & 2.7611 & 2.502406504 & -1.166891317 & 6.356112521 & -2.963903945 \\
\hline 336 & 5.864306287 & 2.7958 & 2.554135676 & -1.137174469 & 6.487504618 & -2.888423152 \\
\hline 337 & 5.881759579 & 2.8308 & 2.605771495 & -1.106084376 & 6.618659598 & -2.809454316 \\
\hline 338 & 5.899212872 & 2.8659 & 2.657200578 & -1.073578721 & 6.749289469 & -2.726889951 \\
\hline 339 & 5.916666164 & 2.9010 & 2.708297762 & -1.039618107 & 6.879076317 & -2.640629992 \\
\hline 340 & 57 & 0 & 2. & -1.0 & 576 & -2.5 \\
\hline 341 & 5.951572749 & 2.9708 & 2.808935324 & -0.967193996 & 7.134695722 & -2.45667275 \\
\hline 342 & 5.969026042 & 3.0053 & 2.858164748 & -0.928674022 & 7.259738459 & -2.358832015 \\
\hline 343 & 5.986479334 & 3.0392 & 2.906440749 & -0.888588111 & 7.382359503 & -2.257013802 \\
\hline 344 & 6.003932627 & 3.0726 & 2.95357876 & -0.846925081 & 7.502090052 & -2.151189706 \\
\hline 345 & 6.021385919 & 3.1052 & 2.999383874 & -0.803682487 & 7.61843504 & -2.041353517 \\
\hline 346 & 6.038839212 & 3.1368 & 3.043652065 & -0.758867691 & 7.730876244 & -1.927523934 \\
\hline 347 & 6.056292504 & 3.1674 & 3.086171772 & -0.712498894 & 7.838876301 & -1.809747192 \\
\hline 348 & 6.073745797 & 3.1966 & 3.126725857 & -0.664606097 & 7.941883676 & -1.688099487 \\
\hline 349 & 6.091199089 & 3.2243 & 3.165093932 & -0.615231937 & 8.039338587 & -1.56268912 \\
\hline 350 & 6.108652382 & 3.2504 & 3.20105505 & -0.564432372 & 8.130679827 & -1.433658225 \\
\hline 351 & 6.126105675 & 3.2747 & 3.234390718 & -0.512277164 & 8.215352423 & -1.301183996 \\
\hline 352 & 6.143558967 & 3.2970 & 3.264888183 & -0.45885011 & 8.292815985 & -1.165479281 \\
\hline 353 & 6.16101226 & 3.3171 & 3.292343921 & -0.404249003 & 8.362553559 & -1.026792467 \\
\hline 354 & 6.178465552 & 3.3348 & 3.316567226 & -0.348585262 & 8.424080754 & -0.885406565 \\
\hline 355 & 6.195918845 & 3.3501 & 3.337383808 & -0.291983249 & 8.476954871 & -0.741637452 \\
\hline 356 & 6.213372137 & 3.3628 & 3.354639259 & -0.234579229 & 8.520783719 & -0.595831241 \\
\hline 357 & 6.23082543 & 3.3728 & 3.368202285 & -0.176520002 & 8.555233804 & -0.448360805 \\
\hline 358 & 6.248278722 & 3.3800 & 3.377967547 & -0.117961226 & 8.580037569 & -0.299621514 \\
\hline 359 & 6.265732015 & 3.3844 & 3.383858023 & -0.059065461 & 8.594999378 & -0.150026272 \\
\hline 360 & 6.283185307 & 3.3858 & 3.385826772 & $-8.29628 \mathrm{E}-16$ & 8.6 & $-2.10726 \mathrm{E}-15$ \\
\hline
\end{tabular}




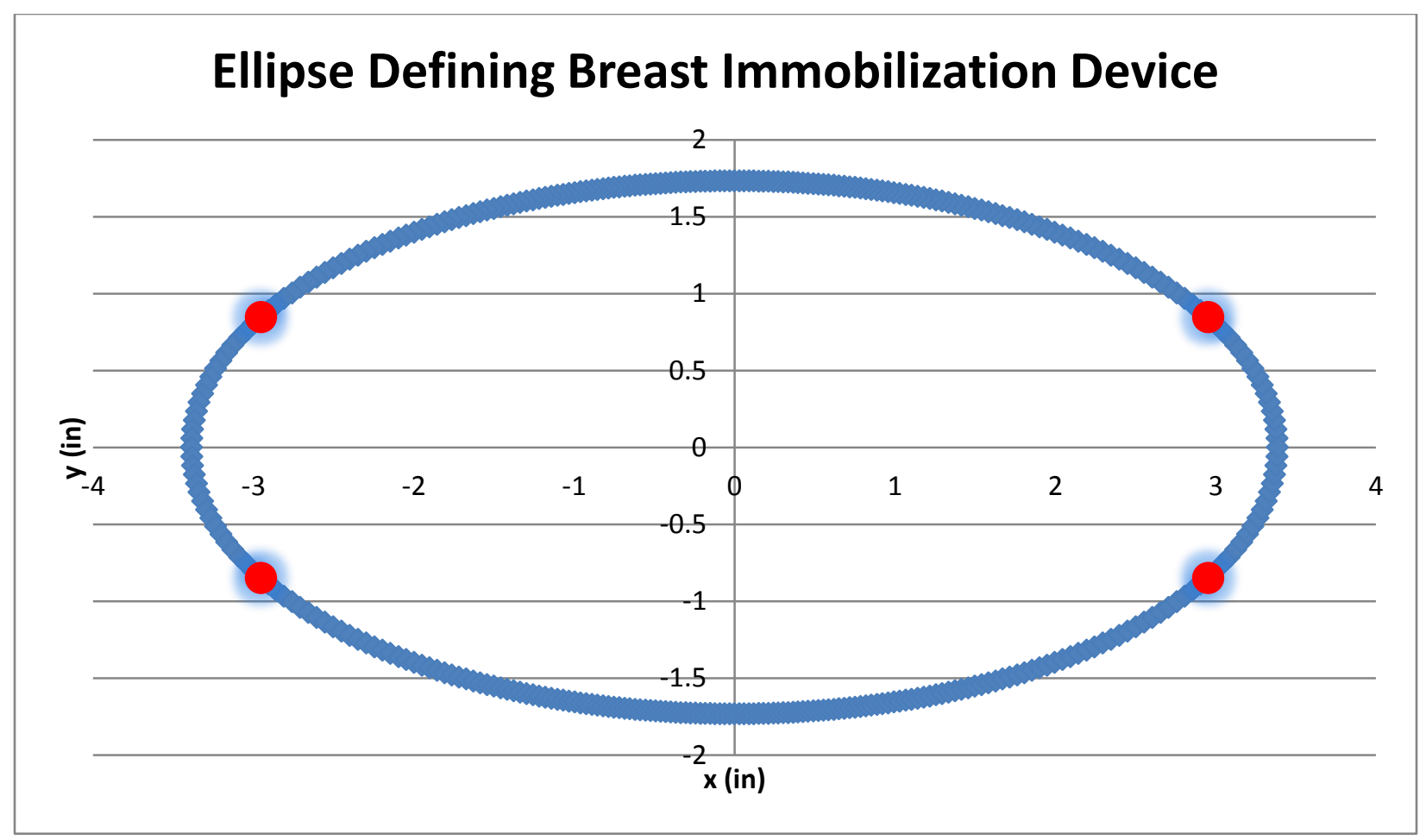

Truncation Point Locations 


\section{APPENDIX B}

(Optimized immobilization device truncation point calculations)

\begin{tabular}{|c|c|c|c|}
\hline $\begin{array}{c}\text { a (1100 mL } \\
\text { breast volume) } \\
(\mathrm{cm})\end{array}$ & $\begin{array}{c}\mathrm{b}(1100 \mathrm{~mL} \\
\text { breast volume }) \\
(\mathrm{cm})\end{array}$ & $\begin{array}{c}\mathrm{b}(840 \mathrm{~mL} \text { breast } \\
\text { volume })(\mathrm{cm})\end{array}$ & $\begin{array}{c}\Delta \text { (distance compression } \\
\text { paddle moves from original } \\
\text { elliptical location) }(\mathrm{cm})\end{array}$ \\
\hline 8.6 & 4.4 & 4.25 & 0.15 \\
\hline
\end{tabular}

\begin{tabular}{|c|c|c|}
\hline $\boldsymbol{w} \mathbf{( c m )}$ & $\mathbf{w} / \mathbf{2} \mathbf{( c m})$ & $\mathbf{A}_{\mathbf{2}}\left(\mathbf{c m}^{\mathbf{2}}\right)$ \\
\hline 0 & 0 & 0 \\
\hline 0.25 & 0.125 & 2.124922535 \\
\hline 0.5 & 0.25 & 4.249380217 \\
\hline 0.75 & 0.375 & 6.372907901 \\
\hline 1 & 0.5 & 8.495039849 \\
\hline 1.25 & 0.625 & 10.61530943 \\
\hline 1.5 & 0.75 & 12.73324884 \\
\hline 1.75 & 0.875 & 14.84838877 \\
\hline 2 & 1 & 16.96025809 \\
\hline 2.25 & 1.125 & 19.0683836 \\
\hline 2.5 & 1.25 & 21.17228961 \\
\hline 2.75 & 1.375 & 23.27149769 \\
\hline 3 & 1.5 & 25.3655263 \\
\hline 3.25 & 1.625 & 27.45389043 \\
\hline 3.5 & 1.75 & 29.53610125 \\
\hline 3.75 & 1.875 & 31.61166575 \\
\hline 4 & 2 & 33.68008631 \\
\hline 4.25 & 2.125 & 35.74086032 \\
\hline 4.5 & 2.25 & 37.79347978 \\
\hline 4.75 & 2.375 & 39.83743078 \\
\hline 5 & 2.5 & 41.87219311 \\
\hline 5.25 & 2.625 & 43.89723972 \\
\hline 5.5 & 2.75 & 45.9120362 \\
\hline 5.75 & 2.875 & 47.91604024 \\
\hline 6 & 3 & 49.90870104 \\
\hline 6.25 & 3.125 & 51.88945868 \\
\hline 6.5 & 3.25 & 53.85774346 \\
\hline 6.75 & 3.375 & 55.81297517 \\
\hline 7 & 3.5 & 57.75456232 \\
\hline 7.25 & 3.625 & 59.68190136 \\
\hline & & \\
\hline
\end{tabular}




\begin{tabular}{|c|c|c|}
\hline 7.5 & 3.75 & 61.59437573 \\
\hline 7.75 & 3.875 & 63.49135494 \\
\hline 8 & 4 & 65.37219351 \\
\hline 8.25 & 4.125 & 67.23622982 \\
\hline 8.5 & 4.25 & 69.08278493 \\
\hline 8.75 & 4.375 & 70.91116115 \\
\hline 9 & 4.5 & 72.72064063 \\
\hline 9.25 & 4.625 & 74.51048368 \\
\hline 9.5 & 4.75 & 76.27992696 \\
\hline 9.75 & 4.875 & 78.02818155 \\
\hline 10 & 5 & 79.75443064 \\
\hline 10.25 & 5.125 & 81.45782711 \\
\hline 10.5 & 5.25 & 83.13749074 \\
\hline 10.75 & 5.375 & 84.79250508 \\
\hline 11 & 5.5 & 86.42191389 \\
\hline 11.25 & 5.625 & 88.02471714 \\
\hline 11.5 & 5.75 & 89.59986645 \\
\hline 11.75 & 5.875 & 91.14625973 \\
\hline 12 & 6 & 92.66273518 \\
\hline 12.25 & 6.125 & 94.1480641 \\
\hline 12.5 & 6.25 & 95.60094266 \\
\hline 12.75 & 6.375 & 97.01998201 \\
\hline 13 & 6.5 & 98.40369661 \\
\hline 13.25 & 6.625 & 99.75049013 \\
\hline 13.5 & 6.75 & 101.0586383 \\
\hline 13.75 & 6.875 & 102.326268 \\
\hline 14 & 7 & 103.5513307 \\
\hline 14.25 & 7.125 & 104.7315692 \\
\hline 14.5 & 7.25 & 105.864475 \\
\hline 14.75 & 7.375 & 106.9472315 \\
\hline 15 & 7.5 & 107.9766377 \\
\hline
\end{tabular}




\section{APPENDIX C}

(Optimized Breast Immobilization Device \& Support Drawings)

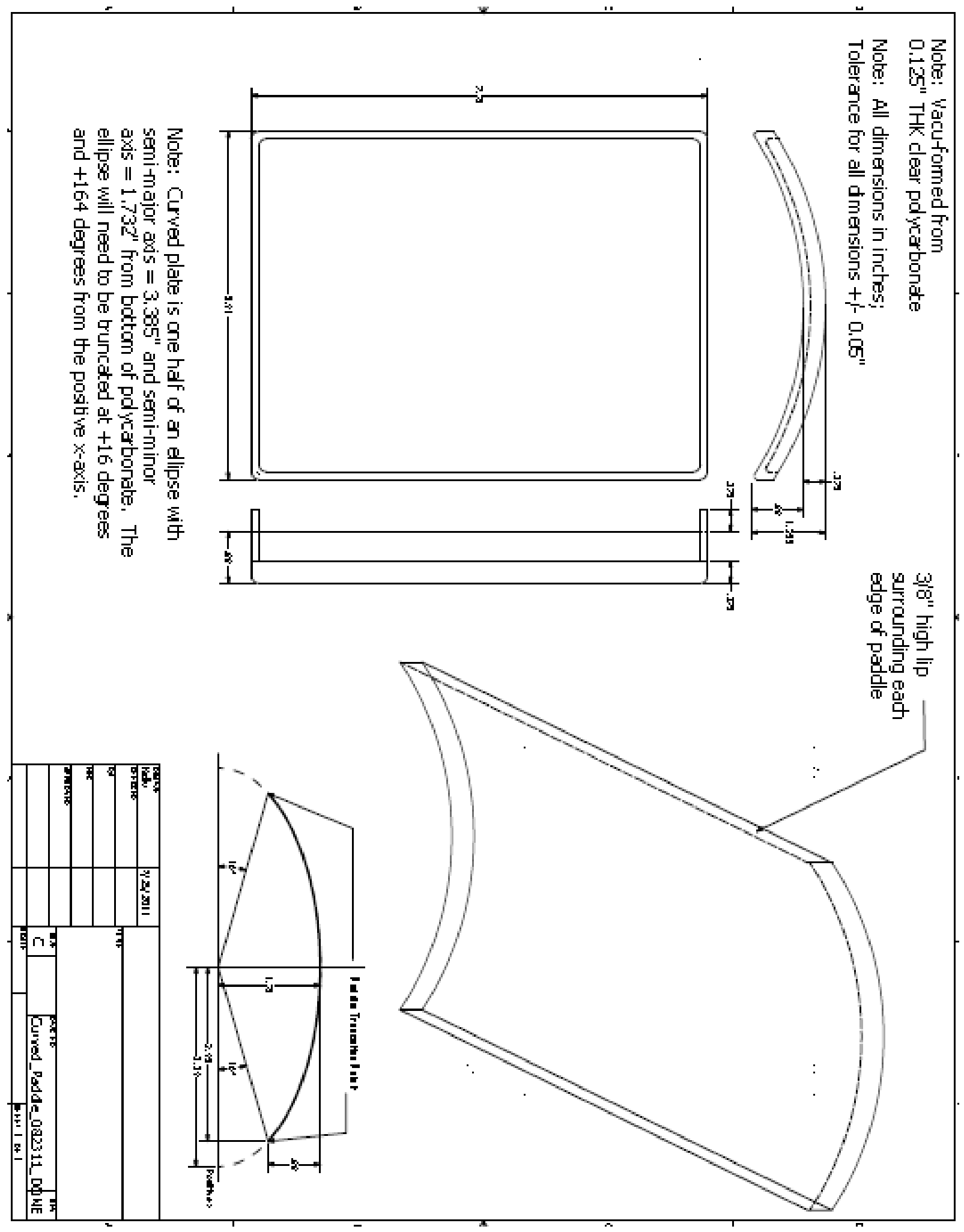


$\underline{\text { Breast Immobilization Device Support System }}$

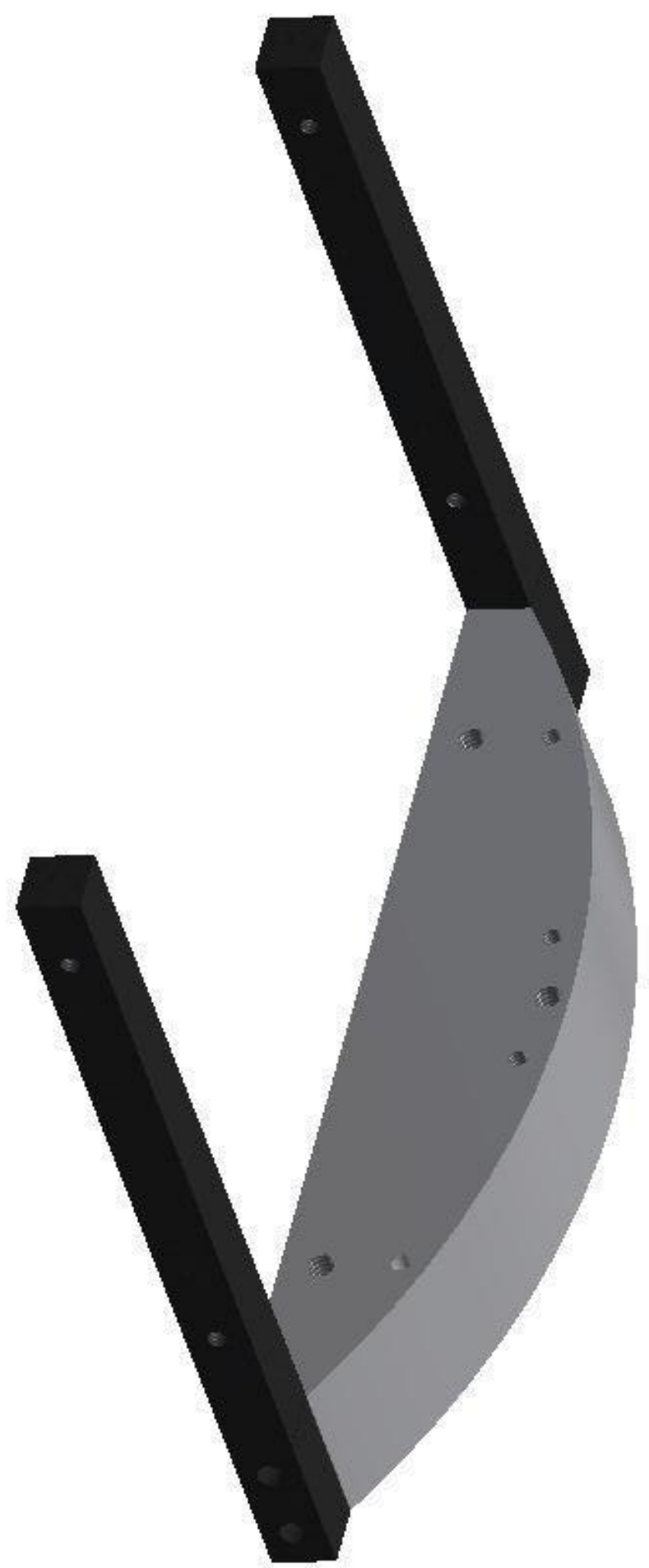




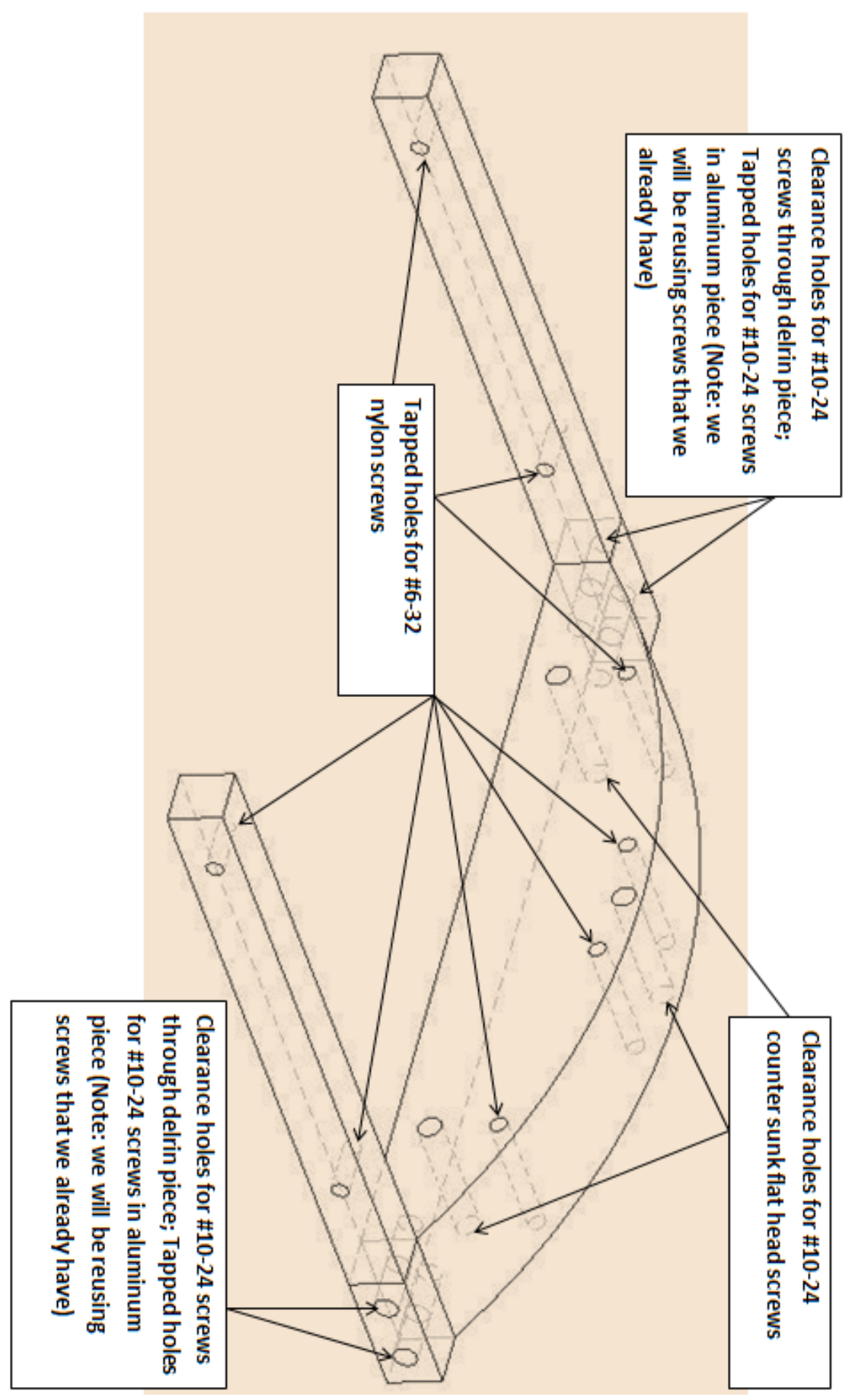




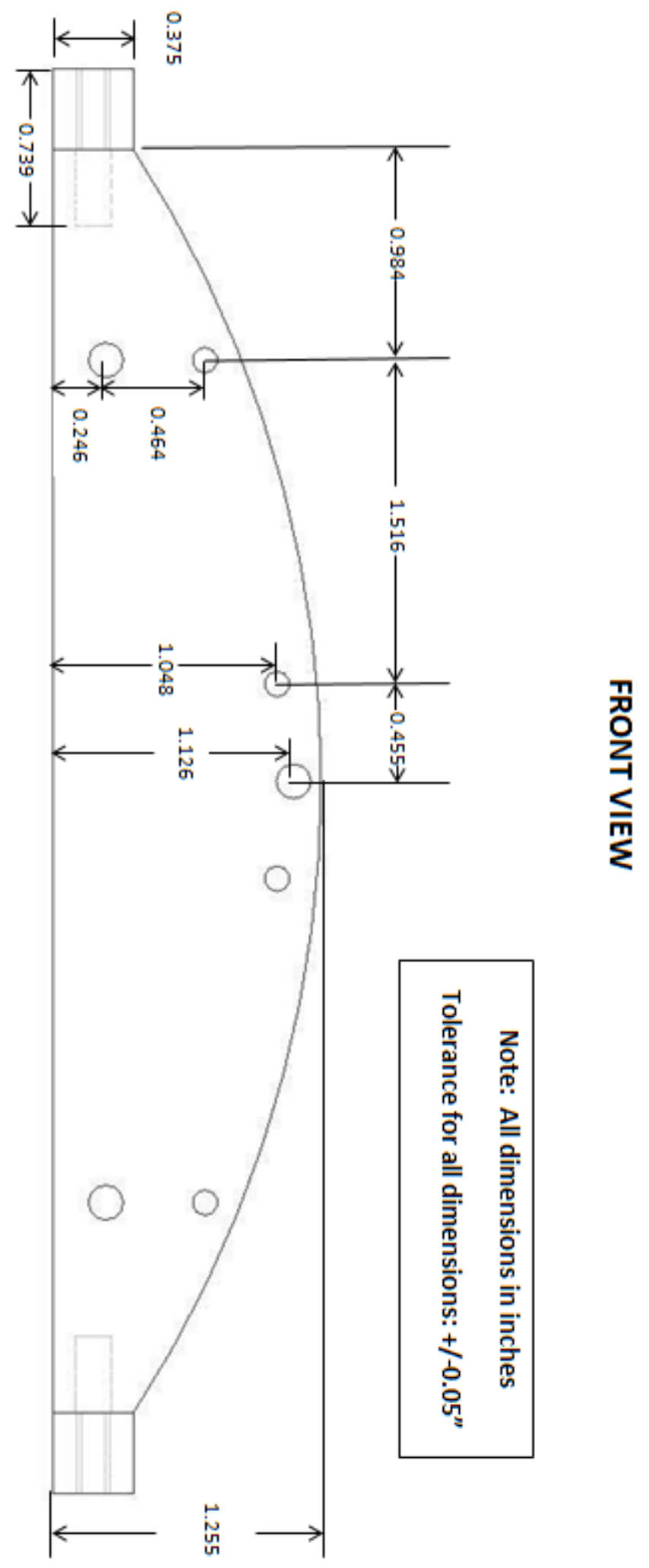



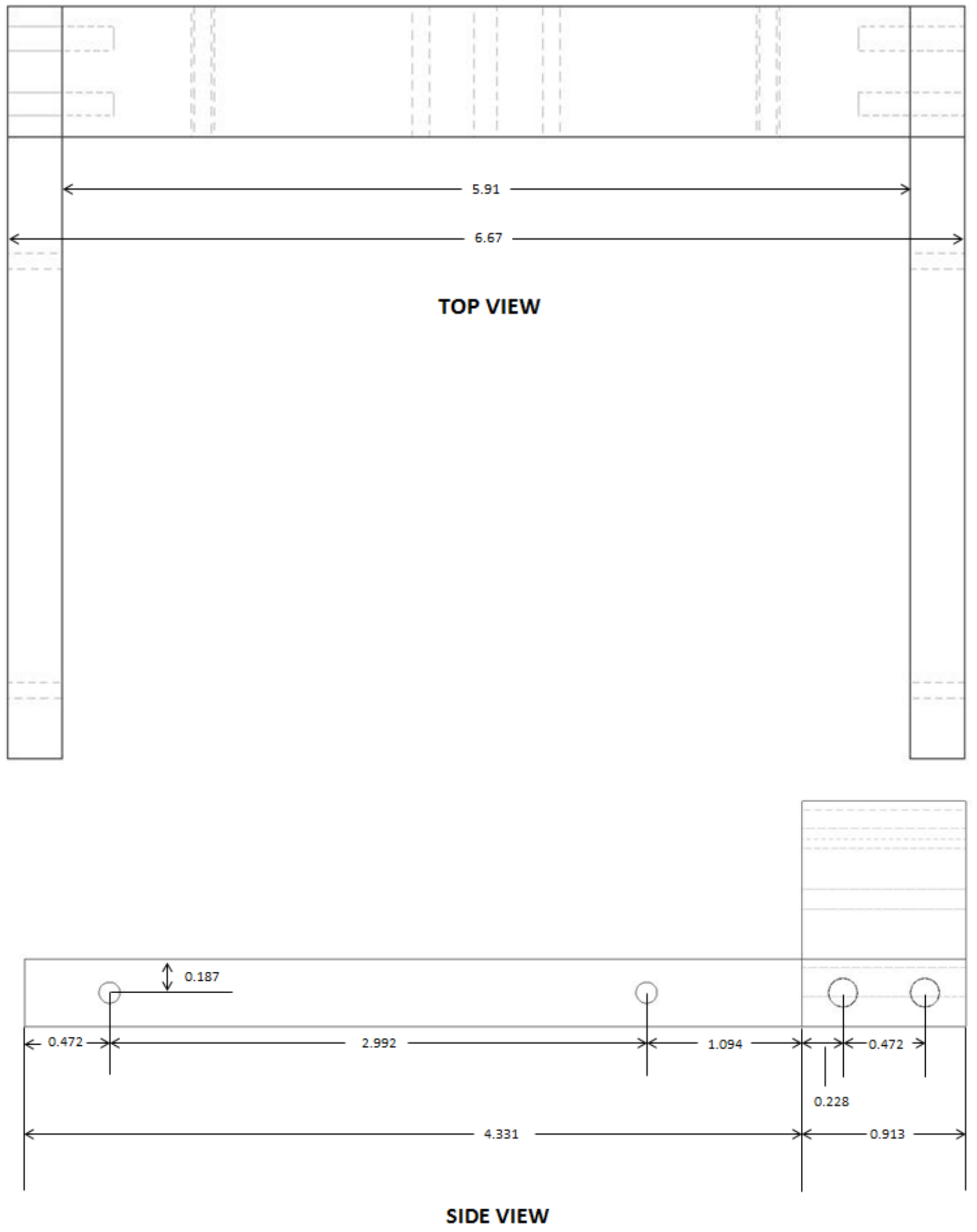


\title{
APPENDIX D
}

\section{(Trajectory Calculations)}

\author{
"Flat Paddle" Trajectory Calculations \\ $a=\quad 12.065 \mathrm{~cm} \quad \mathrm{x} 1 \quad$ Used 1/2 the width of the flat \\ compression paddle used during \\ the pilot DMT study \\ $b=\quad 6.23125 \mathrm{~cm} \quad \mathrm{y} 1 \quad$ Used 1/2 the avg. compressed \\ breast thickness observed during \\ the pilot study $\&$ added $1 / 2$ the \\ thickness of the breast support and \\ compression paddle combined.
}

\begin{tabular}{|c|c|c|c|c|c|}
\hline & & & & & \\
$\boldsymbol{\theta}\left(^{\circ}\right)$ & $\boldsymbol{\theta}$ (radians) & $\mathbf{m}$ & $\mathbf{m o}$ & $\mathbf{x}$ & $\mathbf{y}$ \\
\hline 0 & 0 & 0 & 0 & 0 & 6.23125 \\
\hline 6 & 0.104719755 & -0.1051 & 9.514364 & 0.77959946 & 7.417393393 \\
\hline 12 & 0.20943951 & -0.21256 & 4.70463 & 1.78877588 & 8.415528866 \\
\hline 18 & 0.314159265 & -0.32492 & 3.077684 & 2.983423408 & 9.182033108 \\
\hline 24 & 0.41887902 & -0.44523 & 2.246037 & 4.311330214 & 9.683406205 \\
\hline 30 & 0.523598776 & -0.57735 & 1.732051 & 5.714460399 & 9.897735748 \\
\hline 36 & 0.628318531 & -0.72654 & 1.376382 & 7.13149044 & 9.815654508 \\
\hline 42 & 0.733038286 & -0.9004 & 1.110613 & 8.500489326 & 9.440749827 \\
\hline 48 & 0.837758041 & -1.11061 & 0.900404 & 9.761625235 & 8.78940684 \\
\hline 54 & 0.942477796 & -1.37638 & 0.726543 & 10.85978048 & 7.890092361 \\
\hline 60 & 1.047197551 & -1.73205 & 0.57735 & 11.7469604 & 6.782110748 \\
\hline 66 & 1.151917306 & -2.24604 & 0.445229 & 12.38439098 & 5.513886114 \\
\hline 72 & 1.256637061 & -3.07768 & 0.32492 & 12.74421345 & 4.140845961 \\
\hline 78 & 1.361356817 & -4.70463 & 0.212557 & 12.81070183 & 2.722998733 \\
\hline 84 & 1.466076572 & -9.51436 & 0.105104 & 12.58095026 & 1.322311156 \\
\hline 90 & 1.570796327 & $-1.6 \mathrm{E}+16$ & $6.13 \mathrm{E}-17$ & 12.065 & $7.39071 \mathrm{E}-16$ \\
\hline 96 & 1.675516082 & 9.514364 & -0.1051 & -12.58095026 & 1.322311156 \\
\hline 102 & 1.780235837 & 4.70463 & -0.21256 & -12.81070183 & 2.722998733 \\
\hline 108 & 1.884955592 & 3.077684 & -0.32492 & -12.74421345 & 4.140845961 \\
\hline 114 & 1.989675347 & 2.246037 & -0.44523 & -12.38439098 & 5.513886114 \\
\hline 120 & 2.094395102 & 1.732051 & -0.57735 & -11.7469604 & 6.782110748 \\
\hline 126 & 2.199114858 & 1.376382 & -0.72654 & -10.85978048 & 7.890092361 \\
\hline 132 & 2.303834613 & 1.110613 & -0.9004 & -9.761625235 & 8.78940684 \\
\hline 138 & 2.408554368 & 0.900404 & -1.11061 & -8.500489326 & 9.440749827 \\
\hline
\end{tabular}




\begin{tabular}{|c|c|c|c|c|c|}
\hline 144 & 2.513274123 & 0.726543 & -1.37638 & -7.13149044 & 9.815654508 \\
\hline 150 & 2.617993878 & 0.57735 & -1.73205 & -5.714460399 & 9.897735748 \\
\hline 156 & 2.722713633 & 0.445229 & -2.24604 & -4.311330214 & 9.683406205 \\
\hline 162 & 2.827433388 & 0.32492 & -3.07768 & -2.983423408 & 9.182033108 \\
\hline 168 & 2.932153143 & 0.212557 & -4.70463 & -1.78877588 & 8.415528866 \\
\hline 174 & 3.036872898 & 0.105104 & -9.51436 & -0.77959946 & 7.417393393 \\
\hline 180 & 3.141592654 & $1.23 \mathrm{E}-16$ & $-8.2 E+15$ & $-7.63421 \mathrm{E}-16$ & 6.23125 \\
\hline 186 & 3.246312409 & -0.1051 & 9.514364 & -0.77959946 & -7.417393393 \\
\hline 192 & 3.351032164 & -0.21256 & 4.70463 & -1.78877588 & -8.415528866 \\
\hline 198 & 3.455751919 & -0.32492 & 3.077684 & -2.983423408 & -9.182033108 \\
\hline 204 & 3.560471674 & -0.44523 & 2.246037 & -4.311330214 & -9.683406205 \\
\hline 210 & 3.665191429 & -0.57735 & 1.732051 & -5.714460399 & -9.897735748 \\
\hline 216 & 3.769911184 & -0.72654 & 1.376382 & -7.13149044 & -9.815654508 \\
\hline 222 & 3.874630939 & -0.9004 & 1.110613 & -8.500489326 & -9.440749827 \\
\hline 228 & 3.979350695 & -1.11061 & 0.900404 & -9.761625235 & -8.78940684 \\
\hline 234 & 4.08407045 & -1.37638 & 0.726543 & -10.85978048 & -7.890092361 \\
\hline 240 & 4.188790205 & -1.73205 & 0.57735 & -11.7469604 & -6.782110748 \\
\hline 246 & 4.29350996 & -2.24604 & 0.445229 & -12.38439098 & -5.513886114 \\
\hline 252 & 4.398229715 & -3.07768 & 0.32492 & -12.74421345 & -4.140845961 \\
\hline 258 & 4.50294947 & -4.70463 & 0.212557 & -12.81070183 & -2.722998733 \\
\hline 264 & 4.607669225 & -9.51436 & 0.105104 & -12.58095026 & -1.322311156 \\
\hline 270 & 4.71238898 & $-5.4 \mathrm{E}+15$ & $1.84 \mathrm{E}-16$ & -12.065 & $-2.21721 \mathrm{E}-15$ \\
\hline 276 & 4.817108736 & 9.514364 & -0.1051 & 12.58095026 & -1.322311156 \\
\hline 282 & 4.921828491 & 4.70463 & -0.21256 & 12.81070183 & -2.722998733 \\
\hline 288 & 5.026548246 & 3.077684 & -0.32492 & 12.74421345 & -4.140845961 \\
\hline 294 & 5.131268001 & 2.246037 & -0.44523 & 12.38439098 & -5.513886114 \\
\hline 300 & 5.235987756 & 1.732051 & -0.57735 & 11.7469604 & -6.782110748 \\
\hline 306 & 5.340707511 & 1.376382 & -0.72654 & 10.85978048 & -7.890092361 \\
\hline 312 & 5.445427266 & 1.110613 & -0.9004 & 9.761625235 & -8.78940684 \\
\hline 318 & 5.550147021 & 0.900404 & -1.11061 & 8.500489326 & -9.440749827 \\
\hline 324 & 5.654866776 & 0.726543 & -1.37638 & 7.13149044 & -9.815654508 \\
\hline 330 & 5.759586532 & 0.57735 & -1.73205 & 5.714460399 & -9.897735748 \\
\hline 336 & 5.864306287 & 0.445229 & -2.24604 & 4.311330214 & -9.683406205 \\
\hline 342 & 5.969026042 & 0.32492 & -3.07768 & 2.983423408 & -9.182033108 \\
\hline 348 & 6.073745797 & 0.212557 & -4.70463 & 1.78877588 & -8.415528866 \\
\hline 354 & 6.178465552 & 0.105104 & -9.51436 & 0.77959946 & -7.417393393 \\
\hline 360 & 6.283185307 & $2.45 \mathrm{E}-16$ & $-4.1 \mathrm{E}+15$ & $1.52684 \mathrm{E}-15$ & -6.23125 \\
\hline
\end{tabular}




\section{Optimized Breast Immobilization Device Trajectory Calculations:}
$\mathrm{a}=$
$9.5525 \mathrm{~cm}$
Used $1 / 2$ the width of the $1100 \mathrm{~mL}$ gelatin breast phantom \& added 3/8" to account for height of the lip of the optimized breast immobilization device
$\mathrm{b}=\quad 5.3525 \mathrm{~cm}$
Used $1 / 2$ the thickness of the $1100 \mathrm{~mL}$ gelatin breast phantom \& added 3/8" to account for height of the lip of the optimized breast immobilization device

\begin{tabular}{|c|c|c|c|c|c|c|c|}
\hline $\begin{array}{c}\theta \\
\left({ }^{\circ}\right)\end{array}$ & $\Theta$ (radians) & m & mo & $\mathbf{x 1}$ & y1 & $\times 2$ & y2 \\
\hline 0 & 0 & 0 & 0 & 0 & 5.3525 & 0 & 5.3525 \\
\hline 6 & 0.104719755 & -0.1051 & 9.514364 & -1.76112 & 5.260749581 & -0.56613 & 5.386348147 \\
\hline 12 & 0.20943951 & -0.21256 & 4.70463 & -3.38811 & 5.00451599 & -1.16422 & 5.477218404 \\
\hline 18 & 0.314159265 & -0.32492 & 3.077684 & -4.79191 & 4.630325188 & -1.8184 & 5.596474257 \\
\hline 24 & 0.41887902 & -0.44523 & 2.246037 & -5.9427 & 4.190635092 & -2.54025 & 5.705501601 \\
\hline 29 & 0.506145483 & -0.55431 & 1.804048 & -6.71809 & 3.805159438 & -3.1925 & 5.759423456 \\
\hline 30 & 0.523598776 & -0.57735 & 1.732051 & -6.85496 & 3.727730789 & -3.32789 & 5.764080992 \\
\hline 36 & 0.628318531 & -0.72654 & 1.376382 & -7.56426 & 3.268772694 & -4.16778 & 5.73645934 \\
\hline 42 & 0.733038286 & -0.9004 & 1.110613 & -8.11032 & 2.828001875 & -5.03753 & 5.594748585 \\
\hline 48 & 0.837758041 & -1.11061 & 0.900404 & -8.52855 & 2.410969295 & -5.90889 & 5.32038993 \\
\hline 54 & 0.942477796 & -1.37638 & 0.726543 & -8.84745 & 2.018172782 & -6.75043 & 4.904473336 \\
\hline 60 & 1.047197551 & -1.73205 & 0.57735 & -9.08874 & 1.647488685 & -7.52994 & 4.347413204 \\
\hline 66 & 1.151917306 & -2.24604 & 0.445229 & -9.26844 & 1.295593683 & -8.21652 & 3.658231036 \\
\hline 72 & 1.256637061 & -3.07768 & 0.32492 & -9.39802 & 0.958719347 & -8.78235 & 2.853557241 \\
\hline 78 & 1.361356817 & -4.70463 & 0.212557 & -9.48546 & 0.63301243 & -9.20417 & 1.956405769 \\
\hline 84 & 1.466076572 & -9.51436 & 0.105104 & -9.53598 & 0.314676773 & -9.4645 & 0.994758812 \\
\hline 90 & 1.570796327 & $-1.6 \mathrm{E}+16$ & $6.13 \mathrm{E}-17$ & -9.5525 & 0 & -9.5525 & $5.85162 \mathrm{E}-16$ \\
\hline 96 & 1.675516082 & 9.514364 & -0.1051 & -9.53598 & -0.31467677 & -9.4645 & -0.994758812 \\
\hline 102 & 1.780235837 & 4.70463 & -0.21256 & -9.48546 & -0.63301243 & -9.20417 & -1.956405769 \\
\hline 108 & 1.884955592 & 3.077684 & -0.32492 & -9.39802 & -0.95871935 & -8.78235 & -2.853557241 \\
\hline 114 & 1.989675347 & 2.246037 & -0.44523 & -9.26844 & -1.29559368 & -8.21652 & -3.658231036 \\
\hline 120 & 2.094395102 & 1.732051 & -0.57735 & -9.08874 & -1.64748869 & -7.52994 & -4.347413204 \\
\hline 126 & 2.199114858 & 1.376382 & -0.72654 & -8.84745 & -2.01817278 & -6.75043 & -4.904473336 \\
\hline 132 & 2.303834613 & 1.110613 & -0.9004 & -8.52855 & -2.4109693 & -5.90889 & -5.32038993 \\
\hline 138 & 2.408554368 & 0.900404 & -1.11061 & -8.11032 & -2.82800188 & -5.03753 & -5.594748585 \\
\hline 144 & 2.513274123 & 0.726543 & -1.37638 & -7.56426 & -3.26877269 & -4.16778 & -5.73645934 \\
\hline 150 & 2.617993878 & 0.57735 & -1.73205 & -6.85496 & -3.72773079 & -3.32789 & -5.764080992 \\
\hline
\end{tabular}




\begin{tabular}{|c|c|c|c|c|c|c|c|}
\hline 156 & 2.722713633 & 0.445229 & -2.24604 & -5.9427 & -4.19063509 & -2.54025 & -5.705501601 \\
\hline 162 & 2.827433388 & 0.32492 & -3.07768 & -4.79191 & -4.63032519 & -1.8184 & -5.596474257 \\
\hline 168 & 2.932153143 & 0.212557 & -4.70463 & -3.38811 & -5.00451599 & -1.16422 & -5.477218404 \\
\hline 174 & 3.036872898 & 0.105104 & -9.51436 & -1.76112 & -5.26074958 & -0.56613 & -5.386348147 \\
\hline 180 & 3.141592654 & $1.23 \mathrm{E}-16$ & $-8.2 E+15$ & $-2.1 \mathrm{E}-15$ & -5.3525 & $-6.6 \mathrm{E}-16$ & -5.3525 \\
\hline 186 & 3.246312409 & -0.1051 & 9.514364 & 1.761119 & -5.26074958 & 0.566128 & -5.386348147 \\
\hline 192 & 3.351032164 & -0.21256 & 4.70463 & 3.388109 & -5.00451599 & 1.164219 & -5.477218404 \\
\hline 198 & 3.455751919 & -0.32492 & 3.077684 & 4.791906 & -4.63032519 & 1.818405 & -5.596474257 \\
\hline 204 & 3.560471674 & -0.44523 & 2.246037 & 5.942699 & -4.19063509 & 2.540253 & -5.705501601 \\
\hline 211 & 3.682644722 & -0.60086 & 1.664279 & 6.986181 & -3.65044128 & 3.464758 & -5.766325784 \\
\hline 216 & 3.769911184 & -0.72654 & 1.376382 & 7.564261 & -3.26877269 & 4.167782 & -5.73645934 \\
\hline 222 & 3.874630939 & -0.9004 & 1.110613 & 8.110318 & -2.82800188 & 5.037534 & -5.594748585 \\
\hline 228 & 3.979350695 & -1.11061 & 0.900404 & 8.528546 & -2.4109693 & 5.908892 & -5.32038993 \\
\hline 234 & 4.08407045 & -1.37638 & 0.726543 & 8.847449 & -2.01817278 & 6.750428 & -4.904473336 \\
\hline 240 & 4.188790205 & -1.73205 & 0.57735 & 9.088743 & -1.64748869 & 7.529941 & -4.347413204 \\
\hline 246 & 4.29350996 & -2.24604 & 0.445229 & 9.268 & -1.29559368 & 8.216521 & -3.658231036 \\
\hline 252 & 4.398229715 & -3.07768 & 492 & 9.398016 & -0.95871935 & 8.782346 & -2.853557241 \\
\hline 258 & 4.50294947 & -4.70463 & 0.212557 & 9.485461 & -0.63301243 & 9.204165 & -1.956405769 \\
\hline 264 & 4.607669225 & -9.51436 & 0.105104 & 9.535977 & -0.31467677 & 9.464498 & -0.994758812 \\
\hline 270 & 4.71238898 & $-5.4 \mathrm{E}+15$ & $1.84 \mathrm{E}-16$ & 9.5525 & 0 & 9.5525 & $-1.75548 \mathrm{E}-15$ \\
\hline 276 & 4.817108736 & 9.514364 & -0.1051 & 9.535977 & 0.314676773 & 9.464498 & 0.994758812 \\
\hline 282 & 4.921828491 & 4.70463 & -0.21256 & 9.485461 & 0.63301243 & 9.204165 & 1.956405769 \\
\hline 288 & 5.026548246 & 3.077684 & -0.32492 & 9.398016 & 0.958719347 & 8.782346 & 2.853557241 \\
\hline 294 & 5.131268001 & 2.246037 & -0.44523 & 9.268435 & 1.295593683 & 8.216521 & 3.658231036 \\
\hline 300 & 5.235987756 & 1.732051 & -0.57735 & 9.088743 & 1.647488685 & 7.529941 & 4.347413204 \\
\hline 306 & 5.340707511 & 1.376382 & -0.72654 & 8.847449 & 2.018172782 & 6.750428 & 4.904473336 \\
\hline 312 & 5.445427266 & 1.110613 & -0.9004 & 8.528546 & 2.410969295 & 5.908892 & 5.32038993 \\
\hline 318 & 5.550147021 & 0.900404 & -1.11061 & 8.110318 & 2.828001875 & 5.037534 & 5.594748585 \\
\hline 324 & 5.654866776 & 0.726543 & -1.37638 & 7.564261 & 3.268772694 & 4.167782 & 5.73645934 \\
\hline 330 & 5.759586532 & 0.57735 & -1.73205 & 6.854956 & 3.727730789 & 3.327894 & 5.764080992 \\
\hline 336 & 5.864306287 & 0.445229 & -2.24604 & 5.942699 & 4.190635092 & 2.540253 & 5.705501601 \\
\hline 342 & 5.969026042 & 0.32492 & -3.07768 & 4.791906 & 4.630325188 & 1.818405 & 5.596474257 \\
\hline 348 & 6.073745797 & 0.212557 & -4.70463 & 3.388109 & 5.00451599 & 1.164219 & 5.477218404 \\
\hline 354 & 6.178465552 & 0.105104 & -9.51436 & 1.761119 & 5.260749581 & 0.566128 & 5.386348147 \\
\hline 360 & 6.283185307 & $2.45 \mathrm{E}-16$ & $-4.1 E+15$ & 4.18E-15 & 5.3525 & $1.31 \mathrm{E}-15$ & 5.3525 \\
\hline
\end{tabular}




\section{Gamma Camera Trajectories}

$$
\text { Units }=\mathrm{cm}
$$

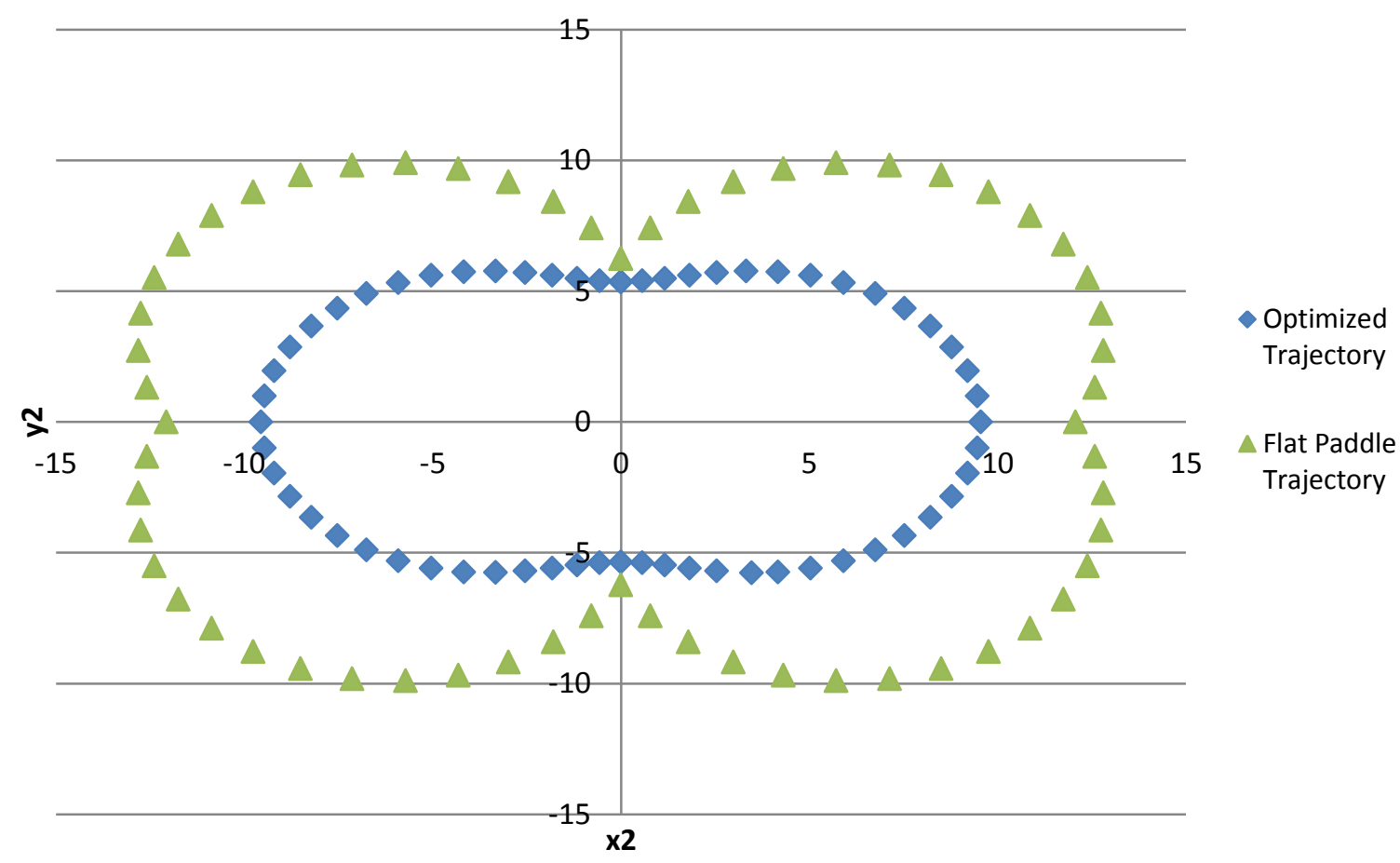




\section{APPENDIX E}

\section{(Image Contrast \& SNR Excel Files; Matlab Source code)}

Trajectory: Optimized Breast Immobilization Device $800 \%$ magnification in Image $\mathrm{J}$

Deep Lesion $(5 \mathrm{~cm})$

\begin{tabular}{|c|c|c|c|c|c|c|c|c|c|c|c|c|c|}
\hline Trial \# & $\begin{array}{c}\text { Lesion } \\
\text { ROI } \\
\text { Area }\end{array}$ & \begin{tabular}{|c|} 
Lesion \\
Mean \\
Pixel \\
Value \\
\end{tabular} & $\begin{array}{c}\text { Lesion } \\
\text { STD } \\
\text { Pixel } \\
\text { Value }\end{array}$ & $\begin{array}{c}\text { BKG } \\
\text { ROI } \\
\text { Area }\end{array}$ & $\begin{array}{c}\text { BKG } \\
\text { Mean } \\
\text { Pixel } \\
\text { Value }\end{array}$ & $\begin{array}{c}\text { BKG } \\
\text { STD } \\
\text { Pixel } \\
\text { Value }\end{array}$ & Slice \# & $\begin{array}{l}\text { x-cord of top } \\
\text { left corner of } \\
\text { ROI (Lesion) }\end{array}$ & $\begin{array}{l}y \text {-cord of top } \\
\text { left corner of } \\
\text { ROI (Lesion) }\end{array}$ & $\begin{array}{c}\text { X-cord of } \\
\text { top left } \\
\text { corner of } \\
\text { ROI (Bkg) }\end{array}$ & $\begin{array}{c}\text { y-cord of } \\
\text { top left } \\
\text { corner of } \\
\text { ROI (Bkg) }\end{array}$ & Contrast & SNR \\
\hline 1 & 16 & 54.685 & 9.821 & 600 & 10.229 & 1.975 & 42 of 94 & 33 & 25 & 44 & 5 & 4.346075 & 22.50937 \\
\hline 2 & 16 & 54.917 & 8.226 & 600 & 10.395 & 1.867 & 42 of 94 & 33 & 25 & 44 & 5 & 4.283021 & 23.84681 \\
\hline 3 & 16 & 52.459 & 9.813 & 600 & 10.093 & 1.982 & 42 of 94 & 33 & 25 & 44 & 5 & 4.197563 & 21.37538 \\
\hline 4 & 16 & 50.748 & 13.678 & 600 & 10.106 & 2.128 & 42 of 94 & 33 & 25 & 44 & 5 & 4.021571 & 19.09868 \\
\hline 5 & 16 & \begin{tabular}{|l|}
49.508 \\
\end{tabular} & 10.54 & 600 & 9.911 & 2.034 & 42 of 94 & 33 & 25 & 44 & 5 & 3.995258 & 19.46755 \\
\hline
\end{tabular}

Trajectory: Optimized Breast Immobilization Device $800 \%$ magnification in Image J

Shallow Lesion $(2 \mathrm{~cm})$

\begin{tabular}{|c|c|c|c|c|c|c|c|c|c|c|c|c|c|}
\hline Trial \# & $\begin{array}{c}\text { Lesion } \\
\text { ROI } \\
\text { Area } \\
\end{array}$ & \begin{tabular}{|c|} 
Lesion \\
Mean \\
Pixel \\
Value \\
\end{tabular} & $\begin{array}{c}\text { Lesion } \\
\text { STD } \\
\text { Pixel } \\
\text { Value } \\
\end{array}$ & $\begin{array}{c}\text { BKG } \\
\text { ROI } \\
\text { Area } \\
\end{array}$ & $\begin{array}{c}\text { BKG } \\
\text { Mean } \\
\text { Pixel } \\
\text { Value } \\
\end{array}$ & $\begin{array}{c}\text { BKG } \\
\text { STD } \\
\text { Pixel } \\
\text { Value } \\
\end{array}$ & Slice \# & $\begin{array}{l}\text { x-cord of top } \\
\text { left corner of } \\
\text { ROI (Lesion) }\end{array}$ & $\begin{array}{l}y \text {-cord of top } \\
\text { left corner of } \\
\text { ROI (Lesion) }\end{array}$ & $\begin{array}{c}\text { X-cord of } \\
\text { top left } \\
\text { corner of } \\
\text { ROI (Bkg) } \\
\end{array}$ & $\begin{array}{c}\mathrm{y} \text {-cord of } \\
\text { top left } \\
\text { corner of } \\
\mathrm{ROI}(\mathrm{Bkg})\end{array}$ & Contrast & SNR \\
\hline 1 & 16 & 71.533 & 15.911 & 600 & 10.769 & 2.037 & 54 of 94 & 65 & 22 & 23 & 7 & 5.642492 & 29.83014 \\
\hline 2 & 16 & 69.131 & 15.64 & 600 & 11.029 & 2.473 & 54 of 94 & 65 & 22 & 23 & 7 & 5.268111 & 23.49454 \\
\hline 3 & 16 & 66.24 & 17.803 & 600 & 10.735 & 2.286 & 54 of 94 & 65 & 22 & 23 & 7 & 5.17047 & 24.2804 \\
\hline 4 & 16 & 58.901 & 13.568 & 600 & 10.703 & 2.032 & 54 of 94 & 65 & 22 & 23 & 7 & 4.503223 & 23.71949 \\
\hline 5 & 16 & 59.075 & 18.506 & 600 & 10.848 & 2.114 & 54 of 94 & 65 & 22 & 23 & 7 & 4.445704 & 22.81315 \\
\hline
\end{tabular}

Trajectory: "Flat Paddle"

$800 \%$ magnification in Image J

Deep Lesion $(5 \mathrm{~cm})$

\begin{tabular}{|c|c|c|c|c|c|c|c|c|c|c|c|c|c|}
\hline Trial \# & $\begin{array}{c}\text { Lesion } \\
\text { ROI } \\
\text { Area } \\
\end{array}$ & $\begin{array}{c}\text { Lesion } \\
\text { Mean } \\
\text { Pixel } \\
\text { Value } \\
\end{array}$ & $\begin{array}{c}\text { Lesion } \\
\text { STD } \\
\text { Pixel } \\
\text { Value } \\
\end{array}$ & $\begin{array}{c}\text { BKG } \\
\text { ROI } \\
\text { Area } \\
\end{array}$ & $\begin{array}{c}\text { BKG } \\
\text { Mean } \\
\text { Pixel } \\
\text { Value } \\
\end{array}$ & $\begin{array}{c}\text { BKG } \\
\text { STD } \\
\text { Pixel } \\
\text { Value } \\
\end{array}$ & Slice \# & $\begin{array}{l}\text { x-cord of top } \\
\text { left corner of } \\
\text { ROI (Lesion) }\end{array}$ & $\begin{array}{l}\text { y-cord of top } \\
\text { left corner of } \\
\text { ROI (Lesion) }\end{array}$ & $\begin{array}{c}\text { X-cord of } \\
\text { top left } \\
\text { corner of } \\
\text { ROI (Bkg) } \\
\end{array}$ & $\begin{array}{c}\mathrm{y} \text {-cord of } \\
\text { top left } \\
\text { corner of } \\
\mathrm{ROI} \text { (Bkg) }\end{array}$ & Contrast & SNR \\
\hline 1 & 16 & 41.22 & 6.906 & 600 & 10.357 & 2.285 & 42 of 94 & 33 & 33 & 44 & 13 & \begin{tabular}{|l|}
2.979917 \\
\end{tabular} & 13.50678 \\
\hline 2 & 16 & 38.882 & 7.615 & 600 & 10.369 & 2.2 & 42 of 94 & 33 & 33 & 44 & 13 & \begin{tabular}{|l|}
2.749831 \\
\end{tabular} & 12.96045 \\
\hline 3 & 16 & 37.002 & 7.889 & 600 & 9.941 & 1.974 & 42 of 94 & 33 & 33 & 44 & 13 & 2.722161 & 13.70871 \\
\hline 4 & 16 & 32.179 & 7.683 & 600 & 9.411 & 1.859 & 42 of 94 & 33 & 33 & 44 & 13 & \begin{tabular}{|l|}
2.419297 \\
\end{tabular} & 12.24744 \\
\hline 5 & 16 & 33.485 & 6.601 & 600 & 9.304 & 1.825 & 42 of 94 & 33 & 33 & 44 & 13 & \begin{tabular}{|l|}
2.59899 \\
\end{tabular} & 13.24986 \\
\hline
\end{tabular}

Trajectory: "Flat Paddle"

$800 \%$ magnification in Image J

Shallow Lesion $(2 \mathrm{~cm})$

\begin{tabular}{|c|c|c|c|c|c|c|c|c|c|c|c|c|c|}
\hline Trial \# & $\begin{array}{c}\text { Lesion } \\
\text { ROI } \\
\text { Area }\end{array}$ & \begin{tabular}{|c|} 
Lesion \\
Mean \\
Pixel \\
Value \\
\end{tabular} & $\begin{array}{l}\text { Lesion } \\
\text { STD } \\
\text { Pixel } \\
\text { Value } \\
\end{array}$ & $\begin{array}{c}\text { BKG } \\
\text { ROI } \\
\text { Area } \\
\end{array}$ & $\begin{array}{c}\text { BKG } \\
\text { Mean } \\
\text { Pixel } \\
\text { Value }\end{array}$ & $\begin{array}{c}\text { BKG } \\
\text { STD } \\
\text { Pixel } \\
\text { Value }\end{array}$ & Slice \# & $\begin{array}{l}\text { x-cord of top } \\
\text { left corner of } \\
\text { ROI (Lesion) }\end{array}$ & $\begin{array}{l}\text { y-cord of top } \\
\text { left corner of } \\
\text { ROI (Lesion) }\end{array}$ & \begin{tabular}{|c|}
$x$-cord of \\
top left \\
corner of \\
ROI (Bkg) \\
\end{tabular} & $\begin{array}{c}\text {-cord of } \\
\text { top left } \\
\text { corner of } \\
\text { ROI (Bkg) } \\
\end{array}$ & Contrast & SNR \\
\hline 1 & 16 & 43.43 & 10.006 & 600 & 9.774 & 1.943 & 54 of 94 & 66 & 28 & 23 & 15 & 3.443421 & 17.32167 \\
\hline 2 & 16 & 46.993 & 10.965 & 600 & 9.807 & 2.195 & 54 of 94 & 66 & 28 & 23 & 15 & 3.791781 & 16.94123 \\
\hline 3 & 16 & 38.993 & 7.624 & 600 & 9.327 & 2.141 & 54 of 94 & 66 & 28 & 23 & 15 & 3.180658 & 13.85614 \\
\hline 4 & 16 & 41.566 & 7.338 & 600 & 9.134 & 2.052 & 54 of 94 & 66 & 28 & 23 & 15 & 3.55069 & 15.80507 \\
\hline 5 & 16 & 43.685 & 11.451 & 600 & 9.14 & 2.006 & 54 of 94 & 66 & 28 & 23 & 15 & 3.77954 & 17.22084 \\
\hline
\end{tabular}




\section{Matlab Source Code (Contrast Plots):}

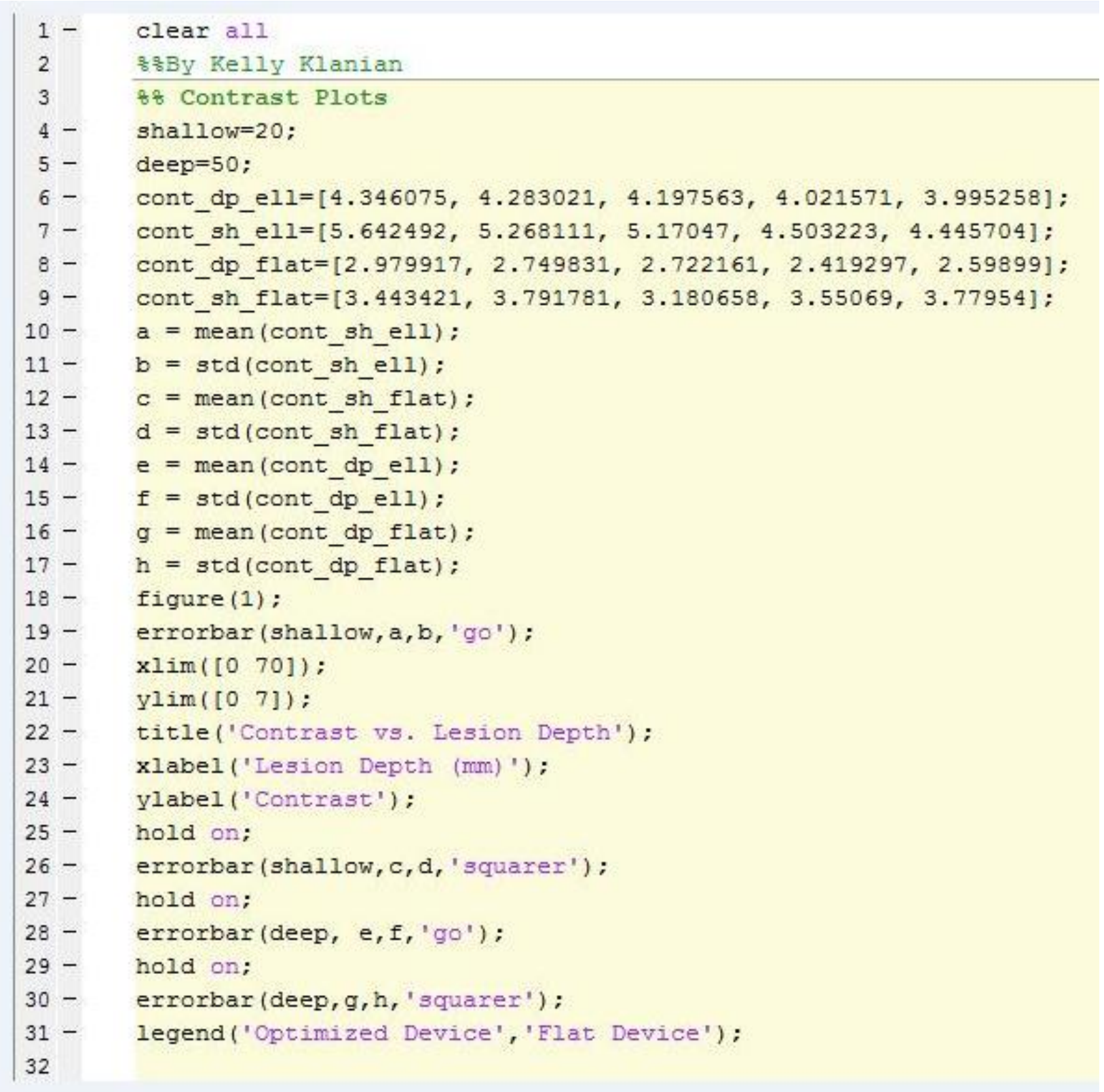




\section{Matlab Source Code (SNR Plots):}

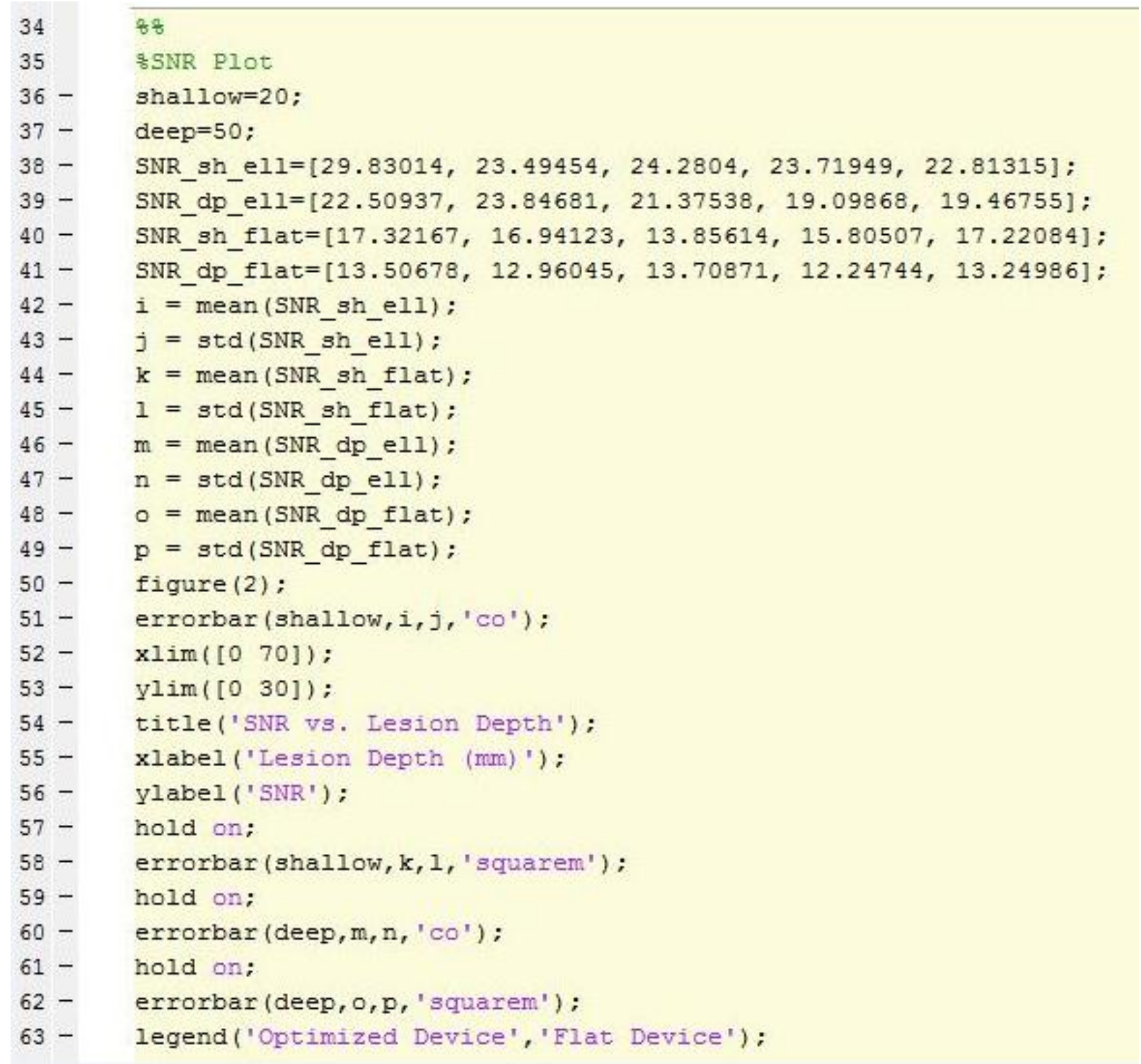




\section{Matlab Source Code (Kruska-Wallis Testing):}

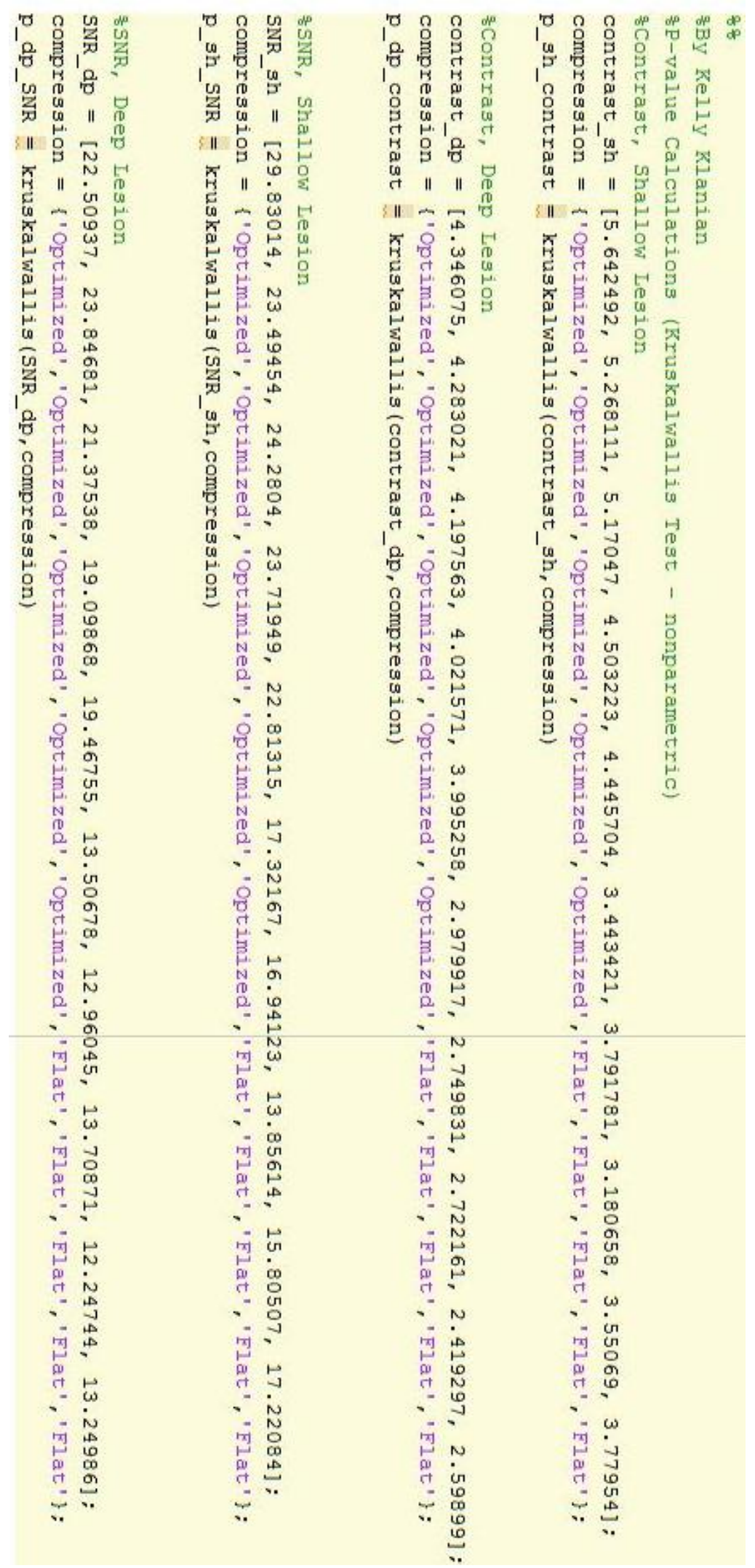




\section{Matlab Source Code (QQ Plots):}

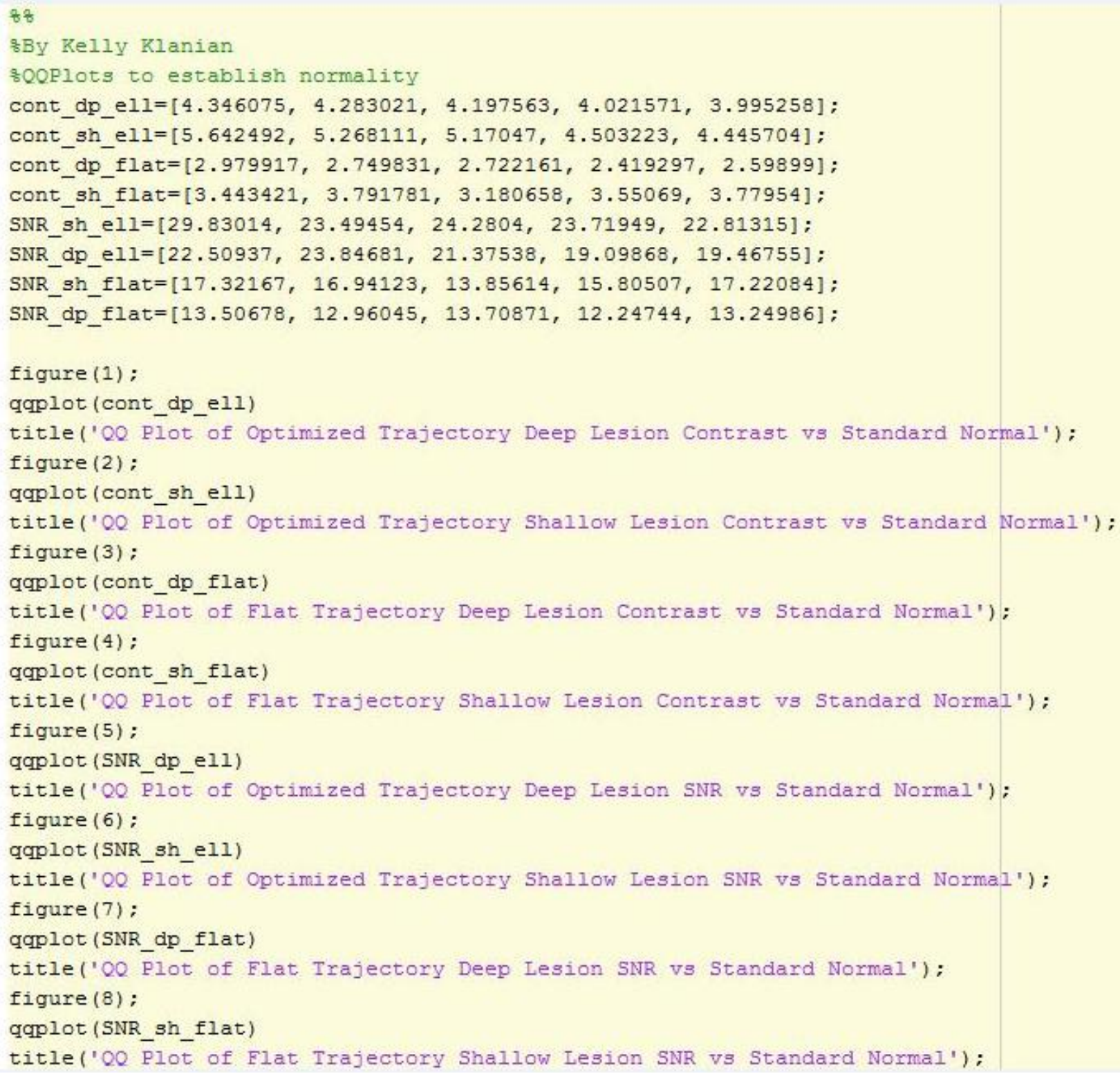




\section{QQ Plots:}
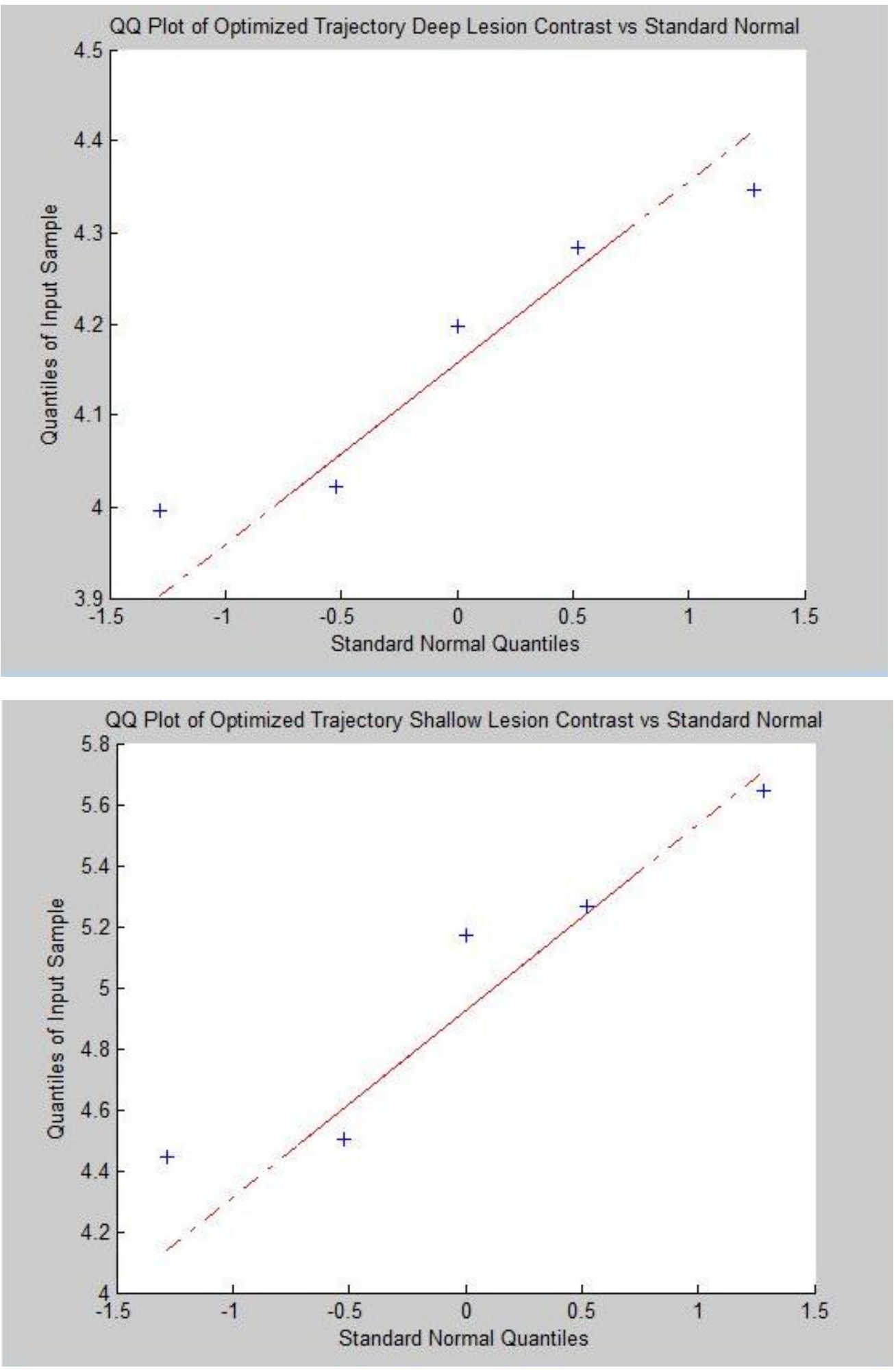

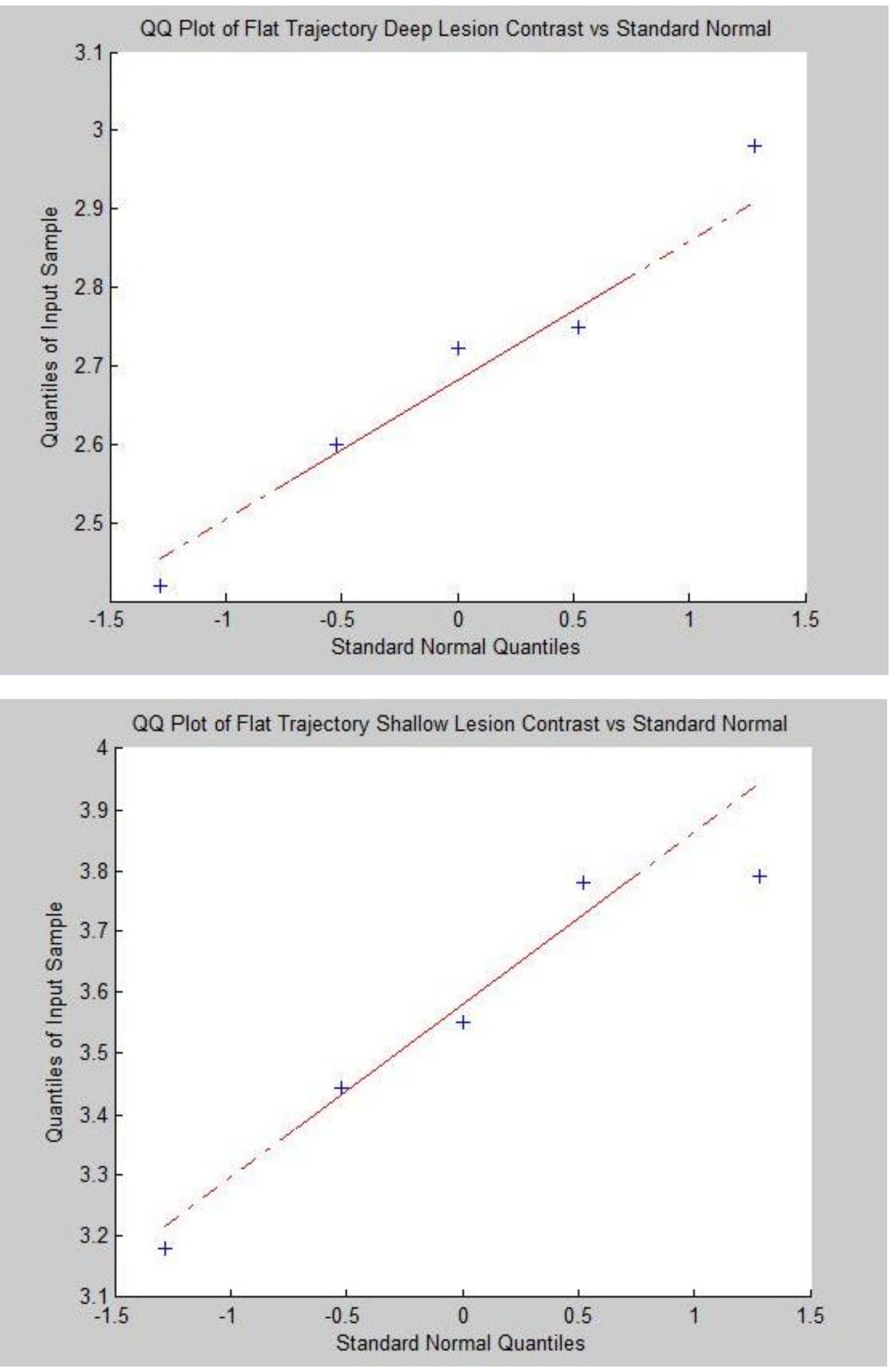

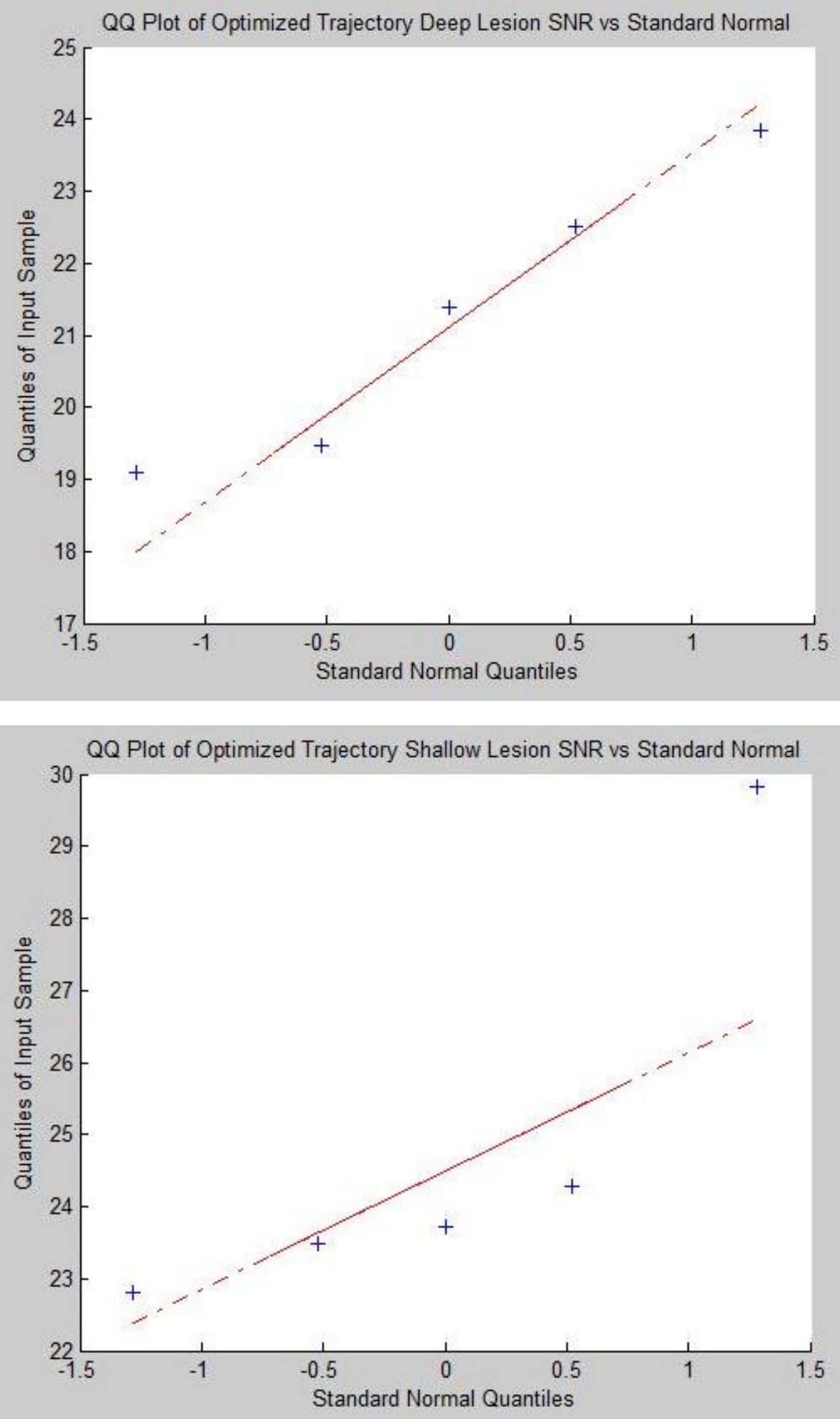

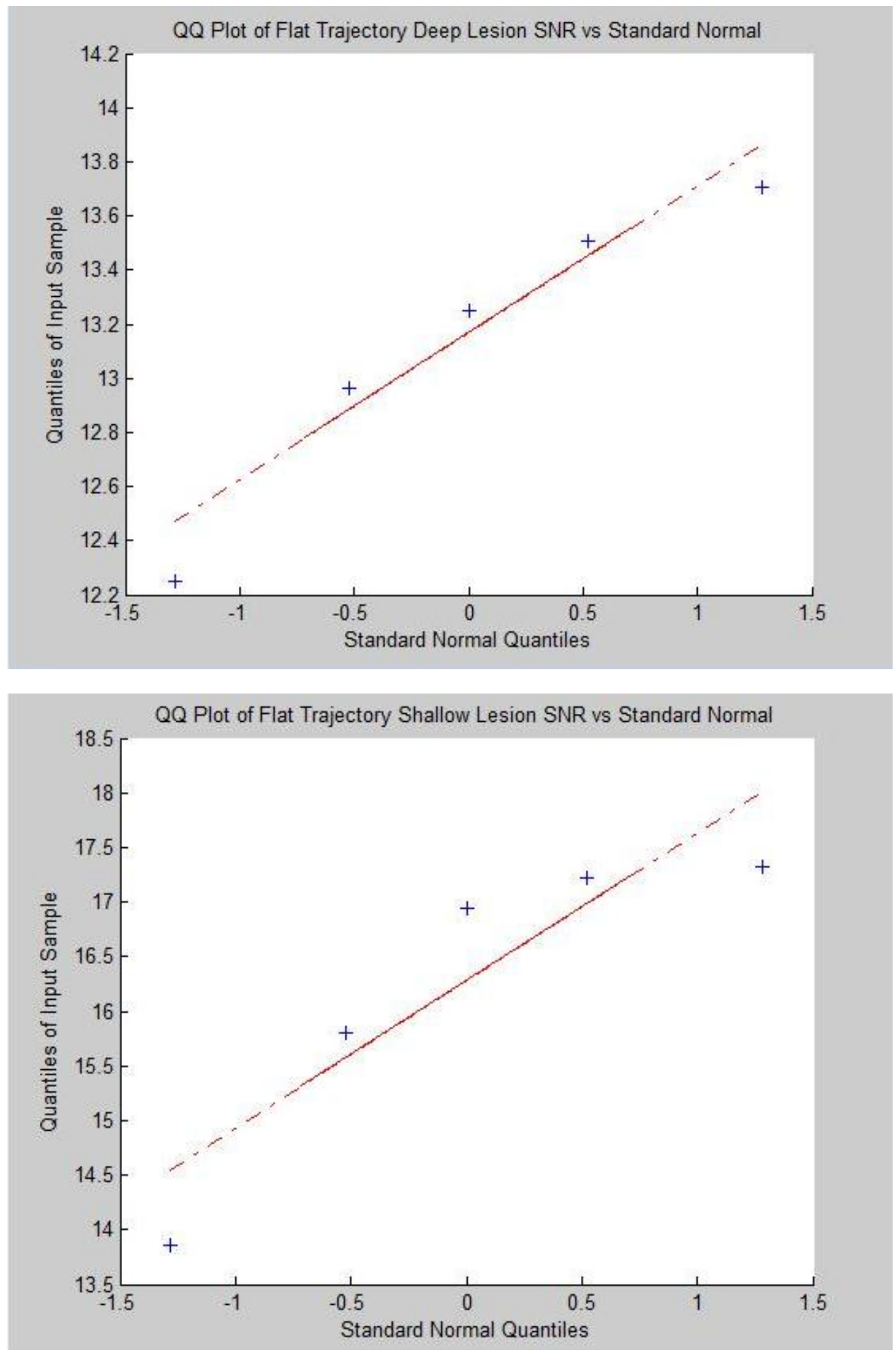


\section{Calculated Variance:}

Trajectory: Optimized Breast Immobilization Device $800 \%$ magnification in Image J

Deep Lesion $(5 \mathrm{~cm})$

\begin{tabular}{|c|c|c|c|c|c|c|}
\hline Trial \# & Contrast & SNR & $\begin{array}{c}\text { STD } \\
\text { Contrast } \\
\end{array}$ & STD SNR & $\begin{array}{l}\text { Variance } \\
\text { Contrast } \\
\end{array}$ & \begin{tabular}{|c} 
Variance \\
SNR
\end{tabular} \\
\hline 1 & 4.346075 & 22.50937 & 0.155799 & 2.009352 & 0.024273 & 4.037497 \\
\hline 2 & 4.283021 & 23.84681 & & & & \\
\hline 3 & 4.197563 & 21.37538 & & & & \\
\hline 4 & 4.021571 & 19.09868 & & & & \\
\hline 5 & 3.995258 & 19.46755 & & & & \\
\hline
\end{tabular}

Trajectory: Optimized Breast Immobilization Device $800 \%$ magnification in Image $J$

Shallow Lesion $(2 \mathrm{~cm})$

\begin{tabular}{|c|c|c|c|c|c|c|}
\hline & & & & & & \\
Trial \# & Contrast & SNR & $\begin{array}{c}\text { STD } \\
\text { Contrast }\end{array}$ & STD SNR & $\begin{array}{c}\text { Variance } \\
\text { Contrast }\end{array}$ & $\begin{array}{c}\text { Variance } \\
\text { SNR }\end{array}$ \\
\hline 1 & 5.642492 & 29.83014 & 0.516621 & 2.845515 & 0.266898 & 8.096955 \\
\hline 2 & 5.268111 & 23.49454 & & & & \\
\hline 3 & 5.17047 & 24.2804 & & & & \\
\hline 4 & 4.503223 & 23.71949 & & & & \\
\hline 5 & 4.445704 & 22.81315 & & & & \\
& & & & & \\
\end{tabular}

Trajectory: "Flat Paddle"

$800 \%$ magnification in Image J

Deep Lesion $(5 \mathrm{~cm})$

\begin{tabular}{|c|c|c|c|c|c|c|}
\hline & & & & & & \\
Trial \# & Contrast & SNR & $\begin{array}{c}\text { STD } \\
\text { Contrast }\end{array}$ & STD SNR & $\begin{array}{c}\text { Variance } \\
\text { Contrast }\end{array}$ & $\begin{array}{c}\text { Variance } \\
\text { SNR }\end{array}$ \\
\hline 1 & 2.979917 & 13.50678 & 0.206245 & 0.569821 & 0.042537 & 0.324696 \\
\hline 2 & 2.749831 & 12.96045 & & & & \\
\hline 3 & 2.722161 & 13.70871 & & & & \\
\hline 4 & 2.419297 & 12.24744 & & & & \\
\hline 5 & 2.59899 & 13.24986 & & & & \\
\cline { 1 - 3 } & & & & & \\
\end{tabular}

Trajectory: "Flat Paddle"

$800 \%$ magnification in Image J

Shallow Lesion $(2 \mathrm{~cm})$

\begin{tabular}{|c|c|c|c|c|c|c|}
\hline & & & & & \\
Trial \# & Contrast & SNR & $\begin{array}{c}\text { STD } \\
\text { Contrast }\end{array}$ & STD SNR & $\begin{array}{c}\text { Variance } \\
\text { Contrast }\end{array}$ & $\begin{array}{c}\text { Variance } \\
\text { SNR }\end{array}$ \\
\hline 1 & 3.443421 & 17.32167 & 0.254419 & 1.457319 & 0.064729 & 2.123777 \\
\hline 2 & 3.791781 & 16.94123 & & & & \\
\hline 3 & 3.180658 & 13.85614 & & & & \\
\hline 4 & 3.55069 & 15.80507 & & & & \\
\hline 5 & 3.77954 & 17.22084 & & & & \\
\hline
\end{tabular}




\section{APPENDIX F}

\section{(Large \& Medium Scaled Optimized Immobilization Device \& Support Drawings)}

Note: Vacu-formed from

0.125 " THK clear polycarbonate

Note: All dimensions in

inches;

Tolerance for all dimensions

$+/-0.05 "$

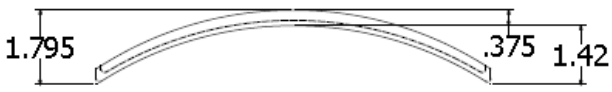

3/8" high lip surrounding

each edge of paddle
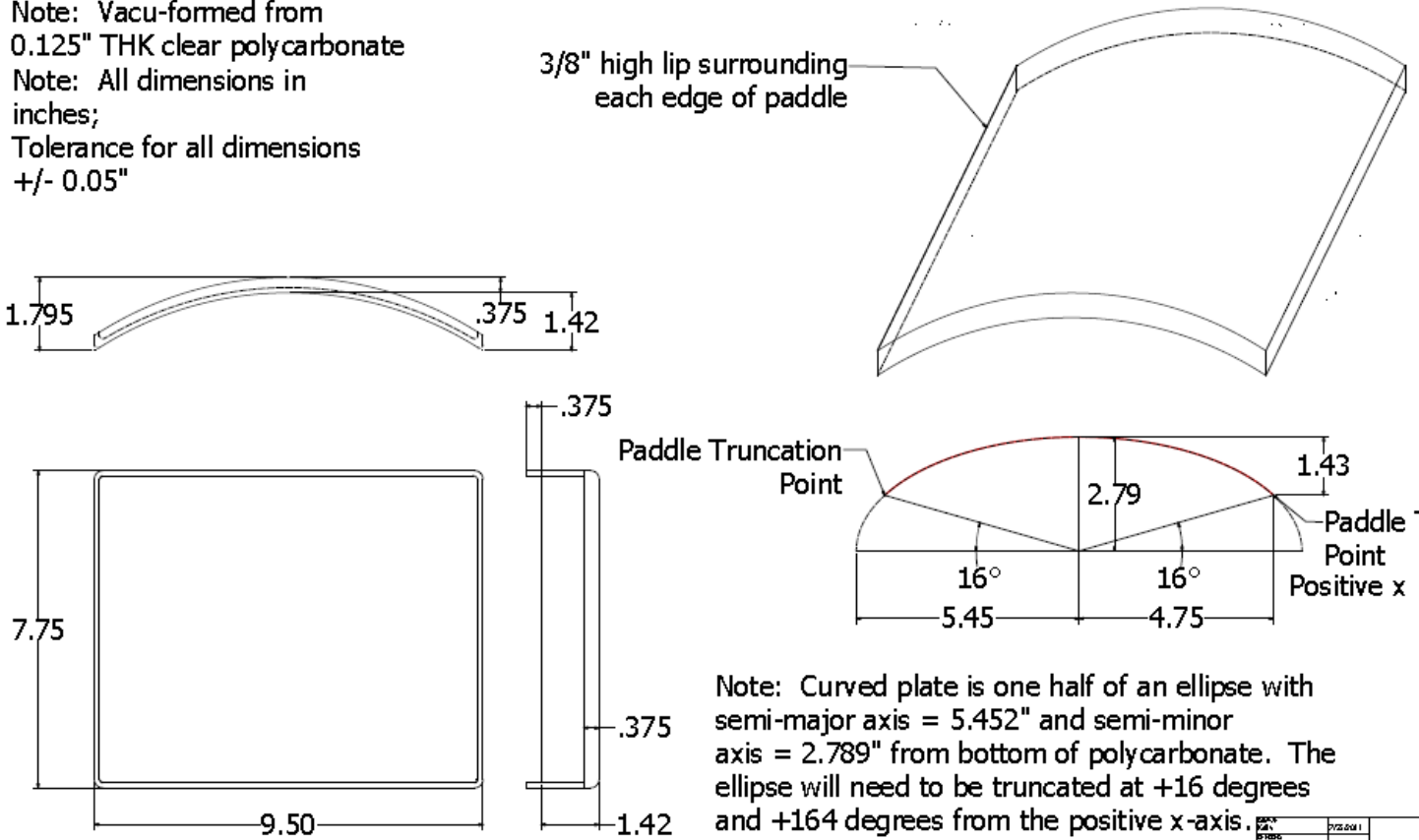

Note: Curved plate is one half of an ellipse with semi-major axis $=5.452 "$ and semi-minor axis $=2.789$ " from bottom of polycarbonate. The ellipse will need to be truncated at +16 degrees and +164 degrees from the positive $x$-axis.

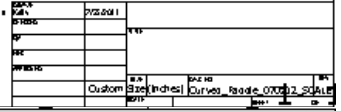

Note: Vacu-formed from

0.125 " THK clear polycarbonate

Note: All dimensions in

inches;

Tolerance for all dimensions

$+/-0.05 "$
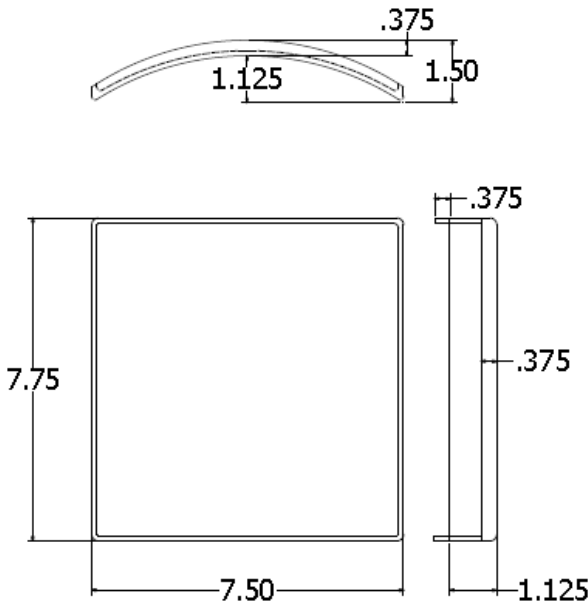

3/8" high lip surrounding each edge of paddle

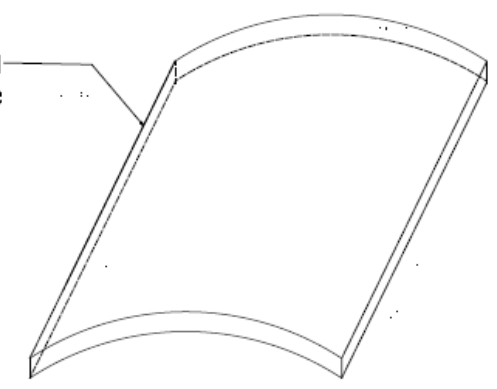

Paddle Truncation-

Point

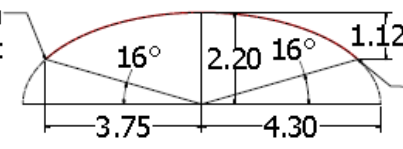

Paddle Truncation Point Positive $x$

Note: Curved plate is one half of an ellipse with semi-major axis $=4.304 "$ and semi-minor axis $=2.202$ " from bottom of polycarbonate. The ellipse will need to be truncated at +16 degrees and +164 degrees from the positive $x$-axis.

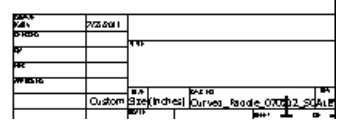




\section{$\underline{\text { Breast Immobilization Device Support System Redesign (Large) }}$}

Clearance hole for \#1032 counter sunk flat head screws

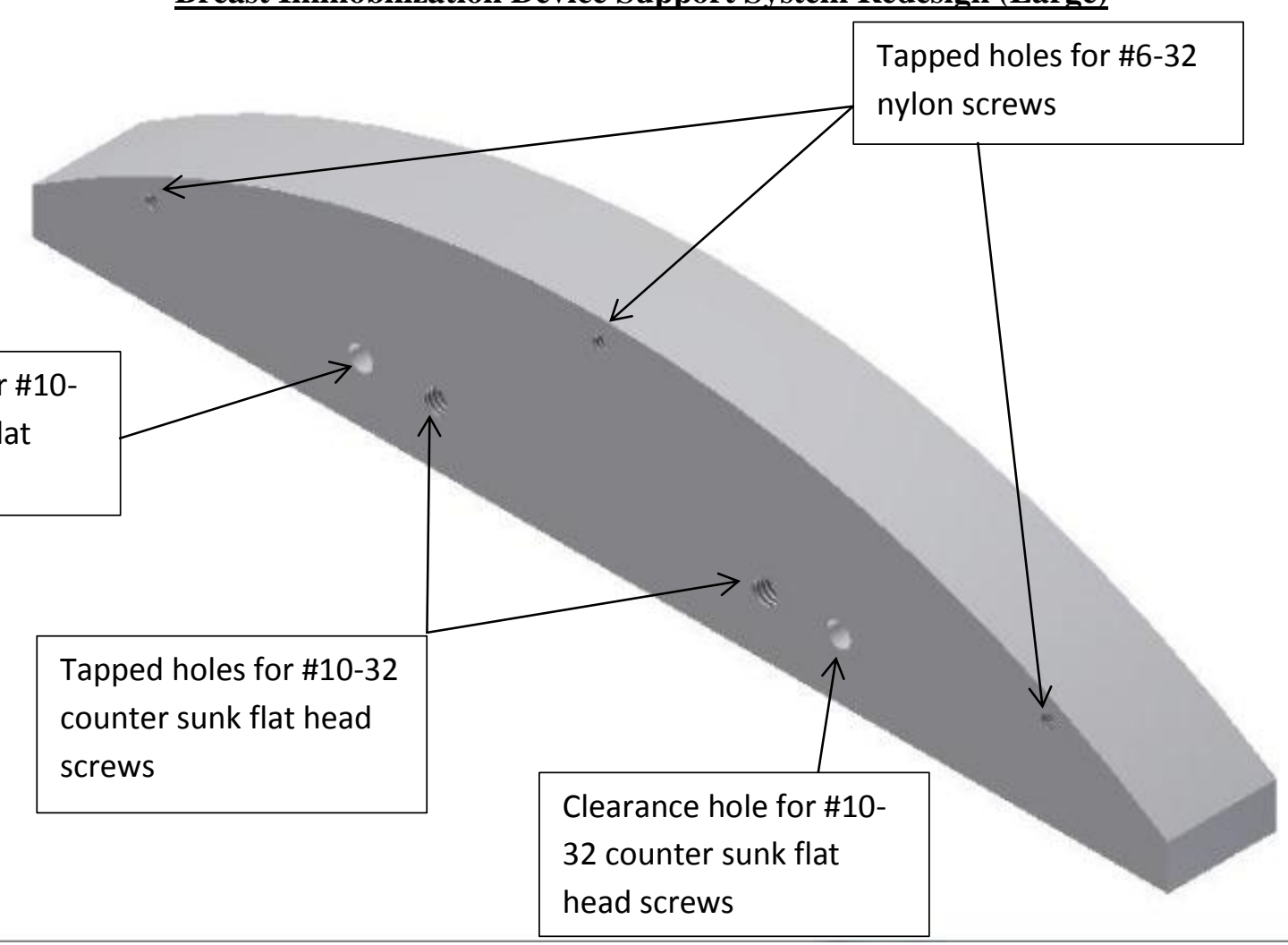




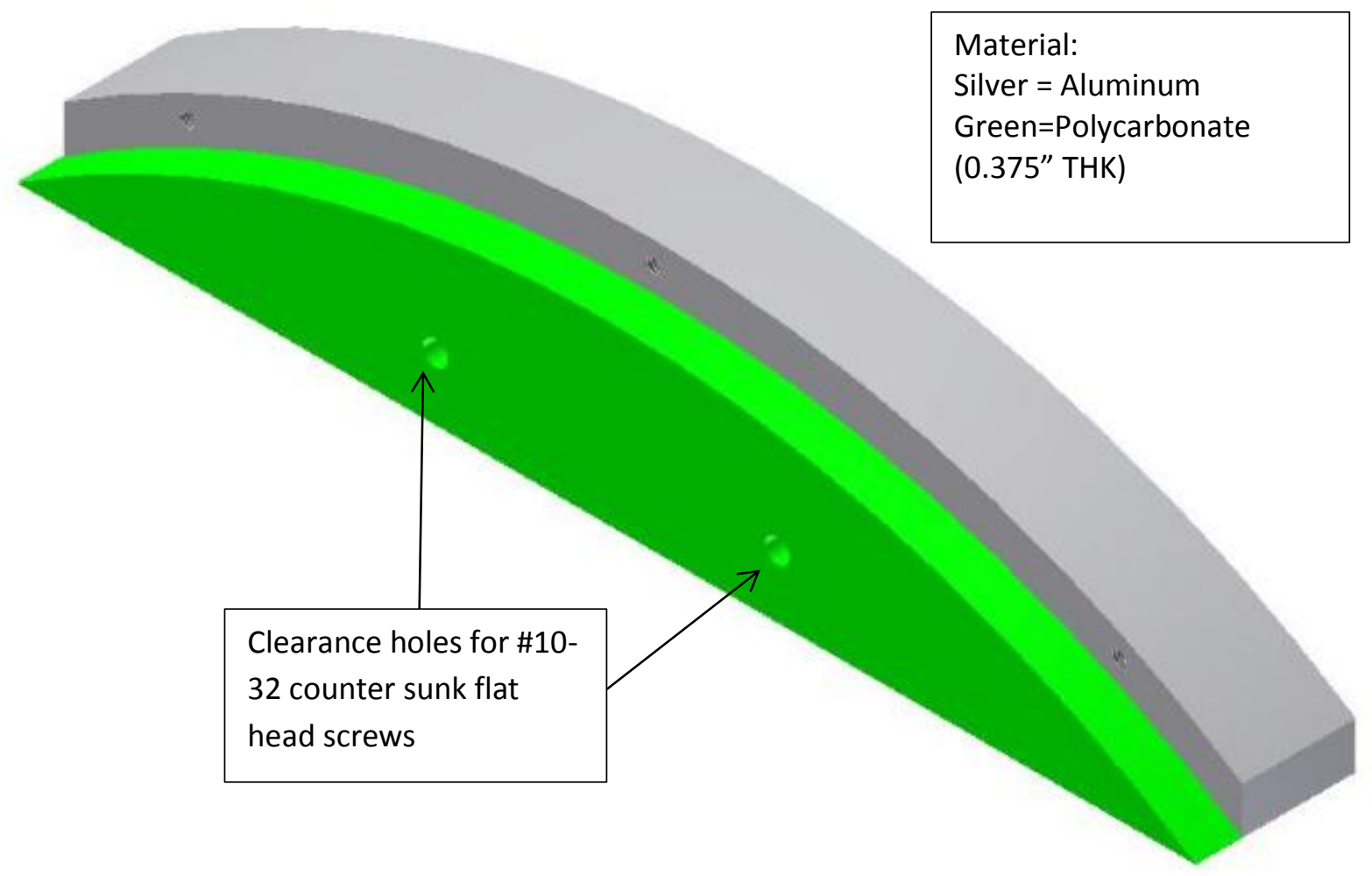




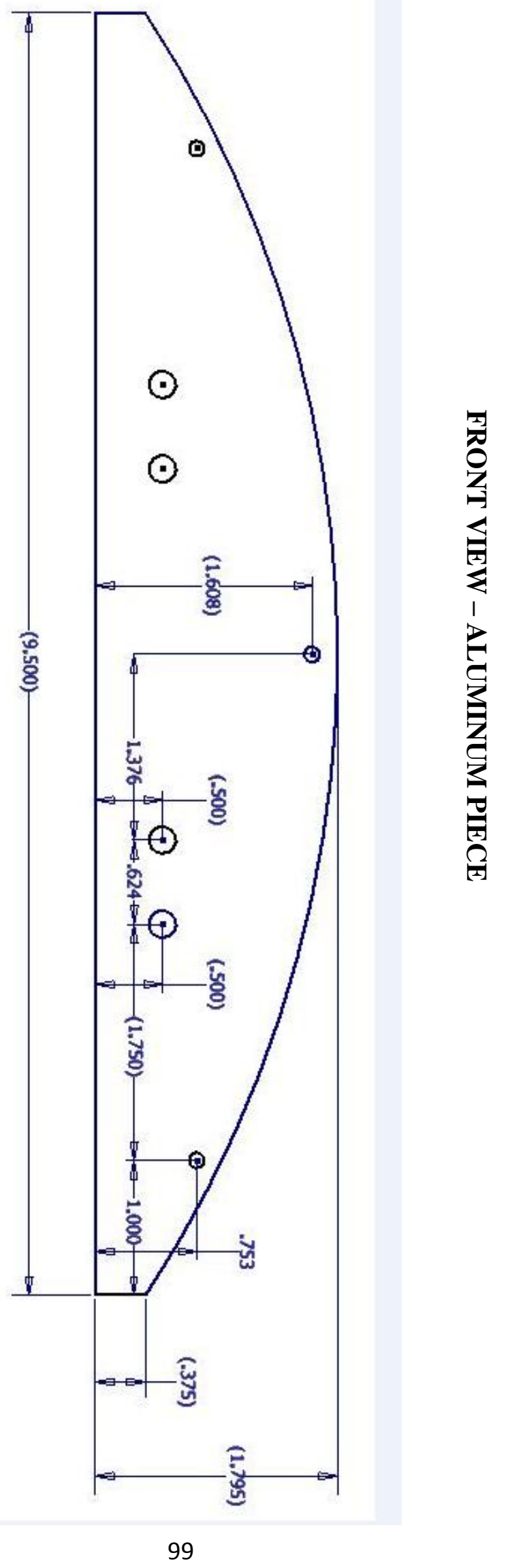




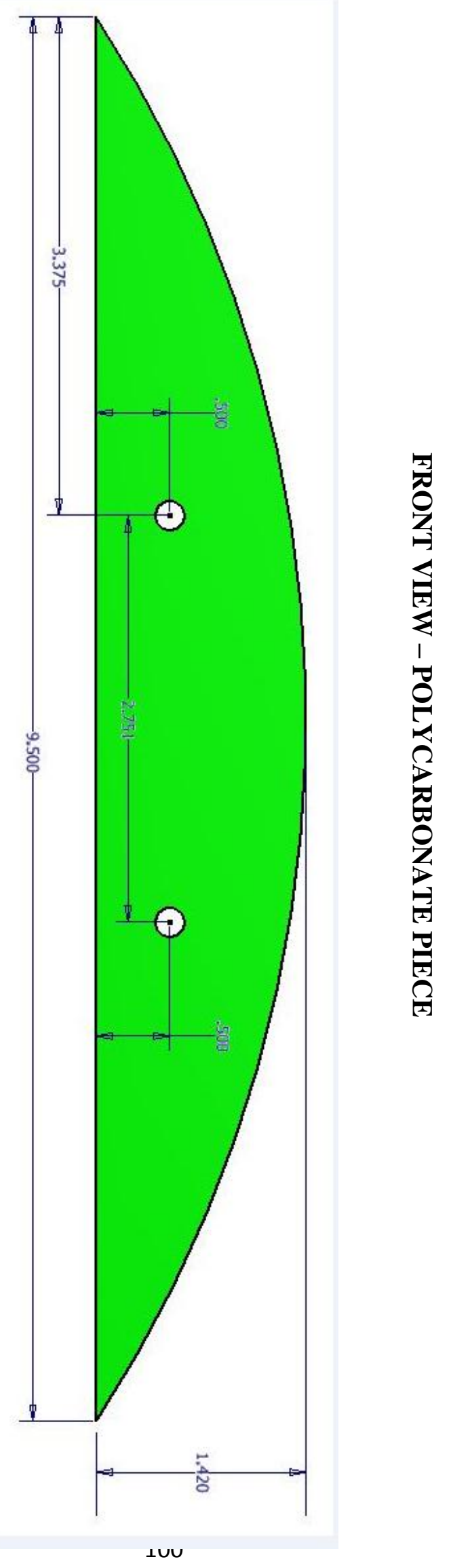


SIDE VIEW - BOTH ALUMINUM AND POLYCARBONATE PIECES

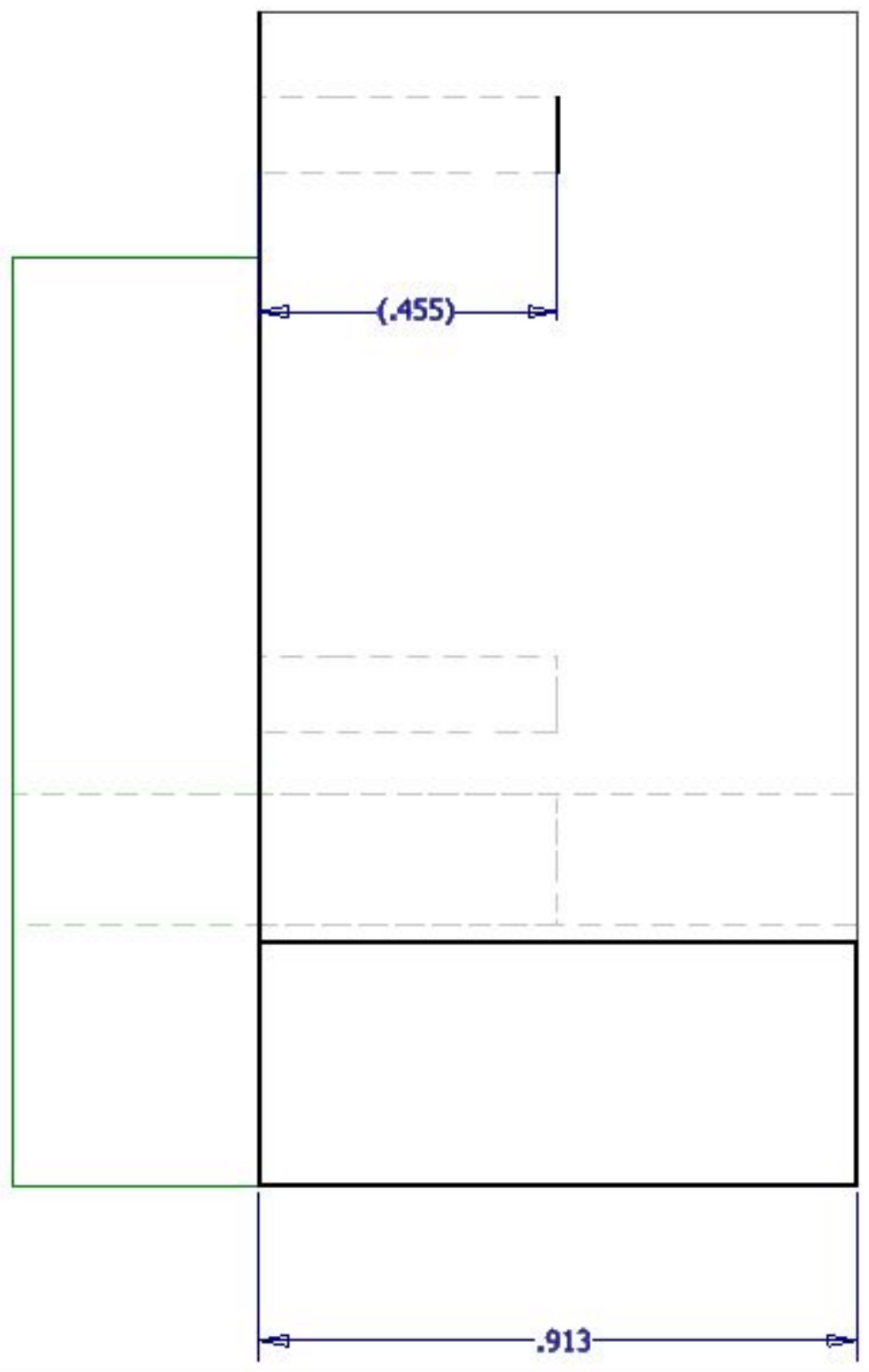




\section{$\underline{\text { Breast Immobilization Device Support System Redesign (Medium) }}$}
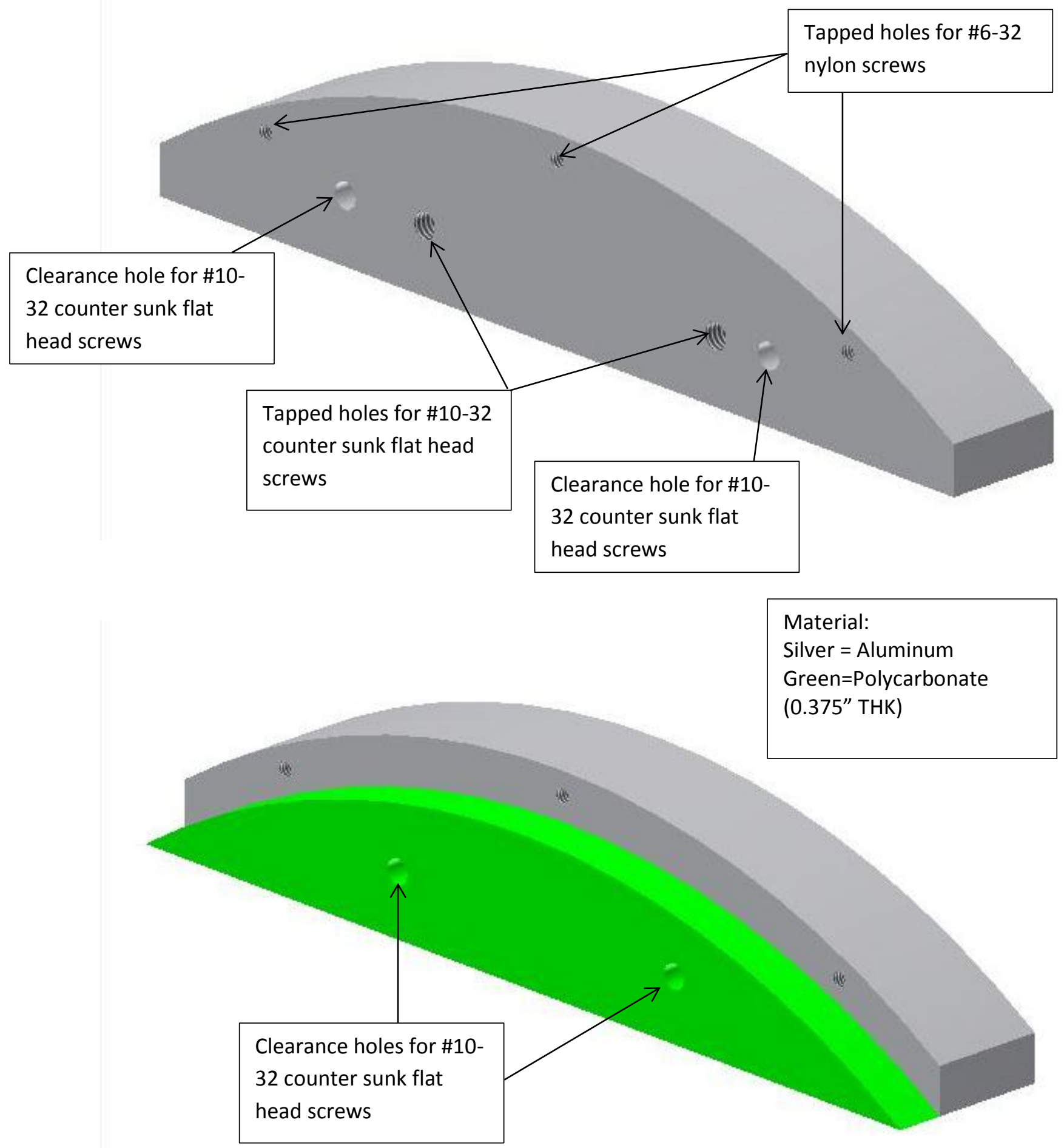


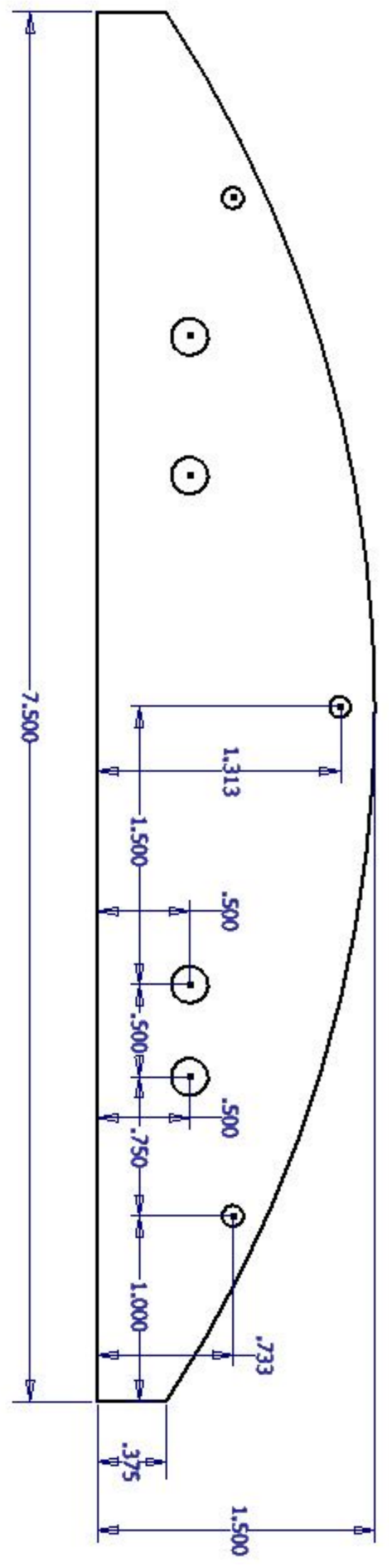

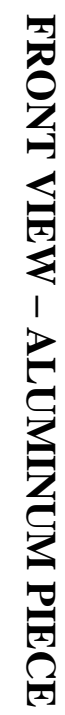




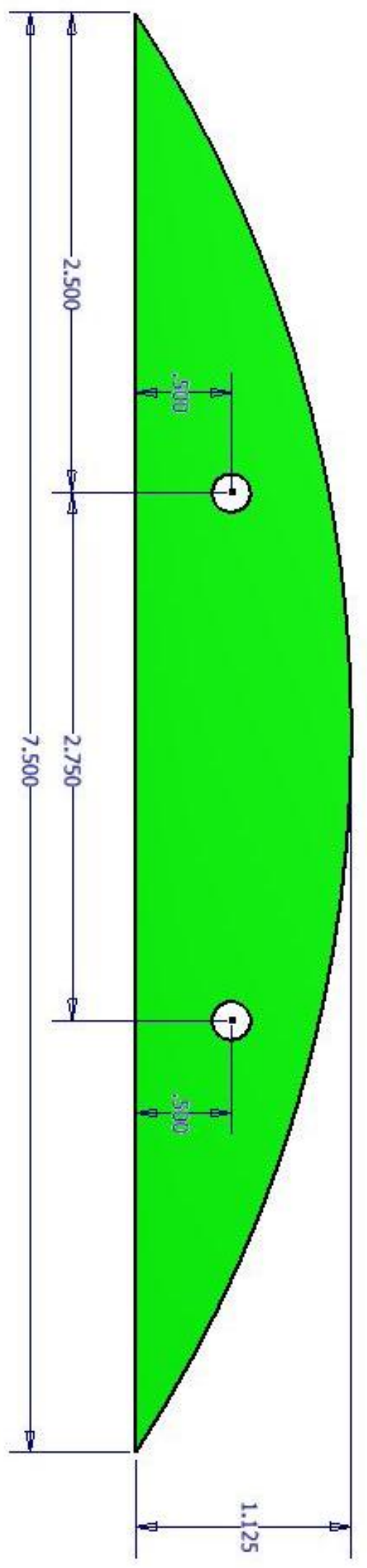

资 
SIDE VIEW - BOTH ALUMINUM AND POLYCARBONATE PIECES

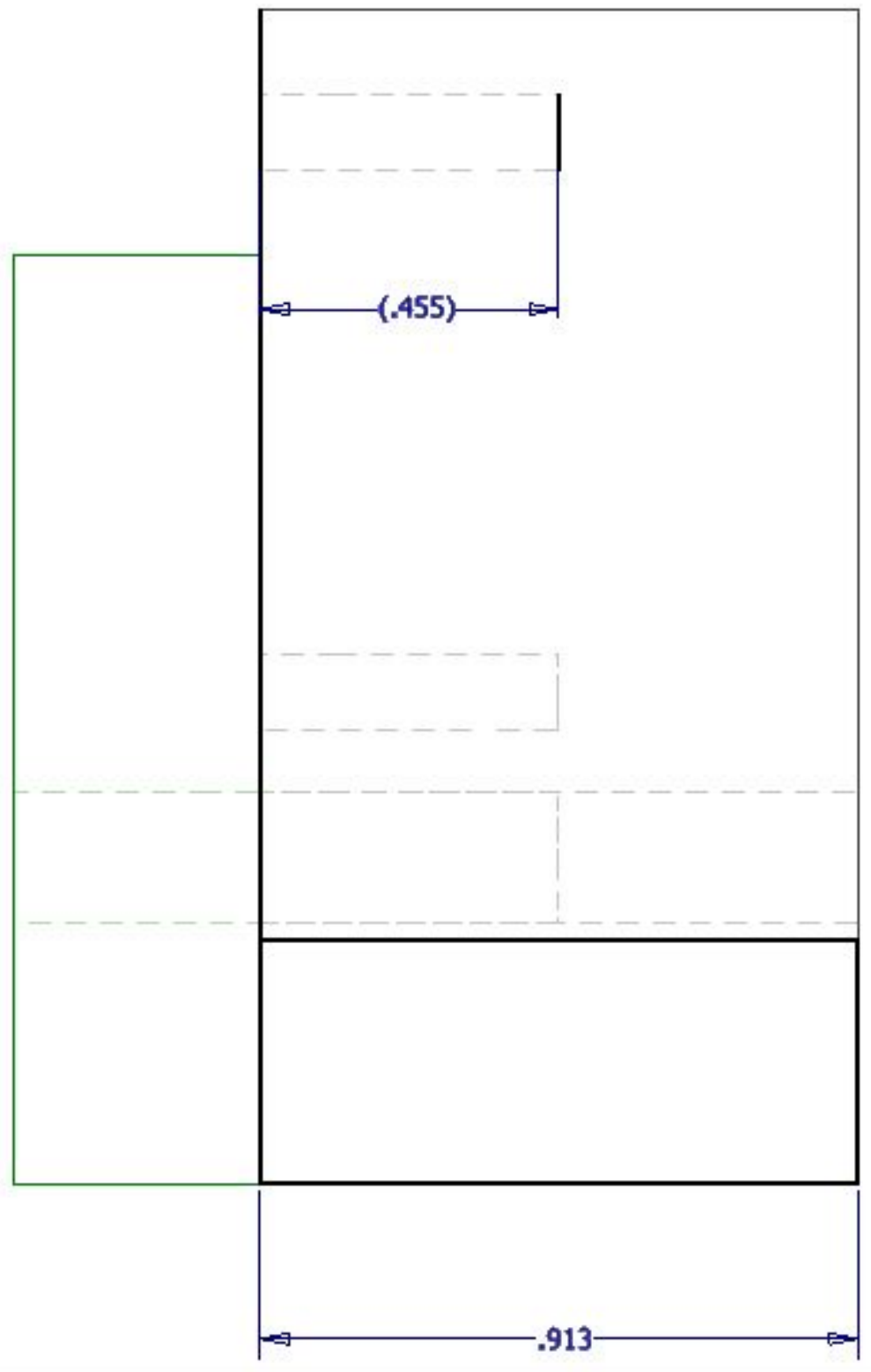




\section{APPENDIX G}

(X-ray detector hinge \& lead brick weight subassembly drawings)

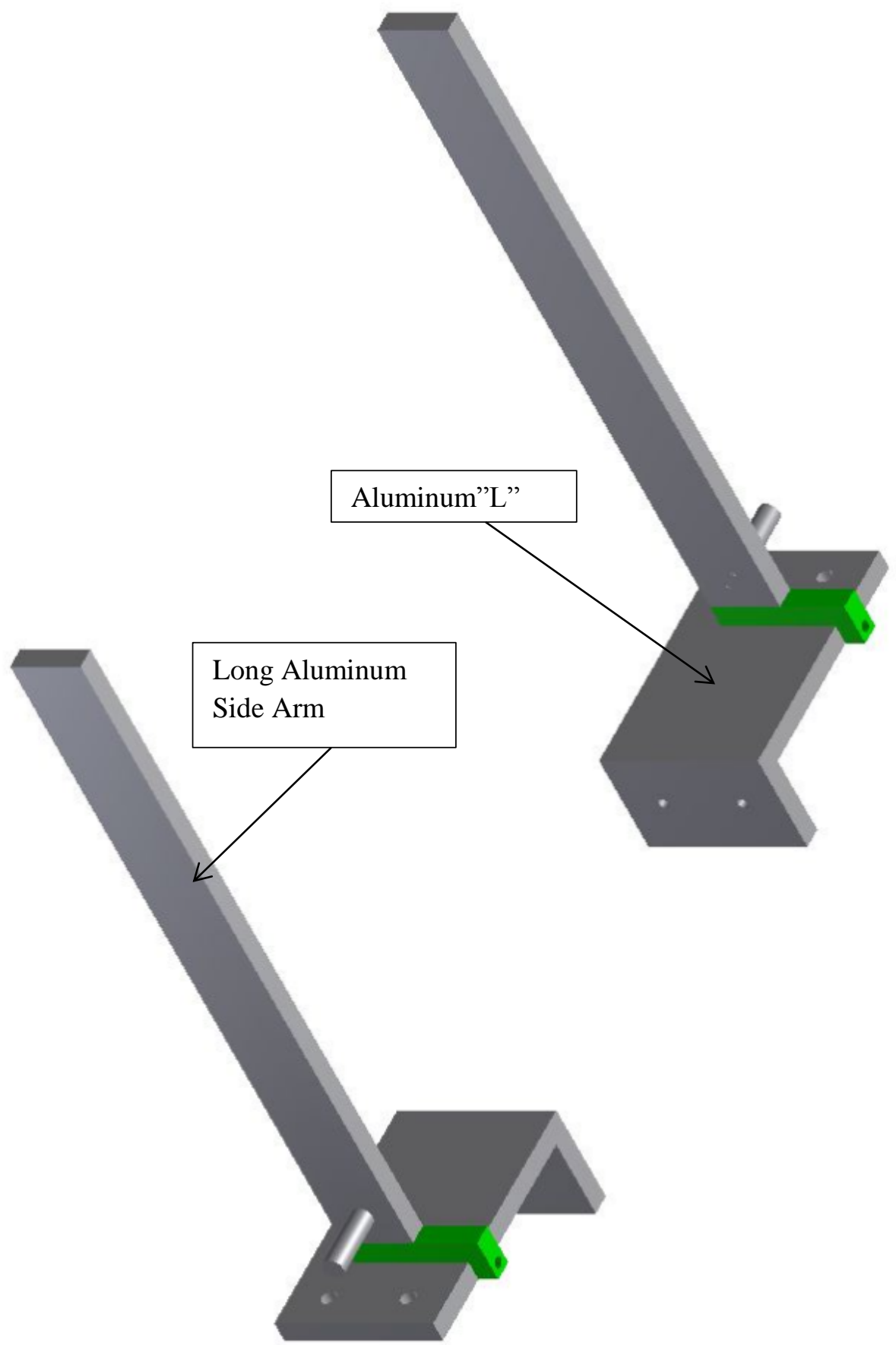




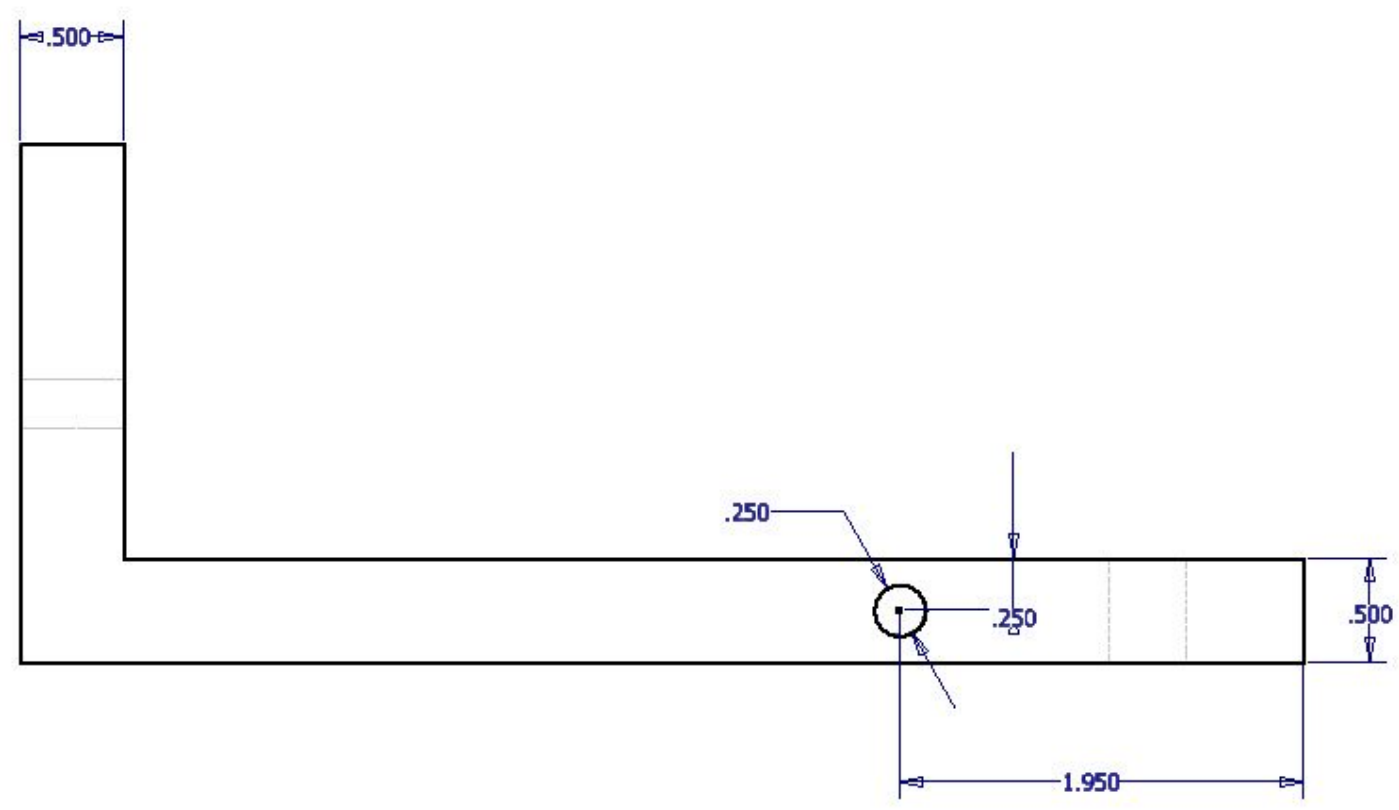

FRONT VIEW - ALUMINUM "L" PIECE

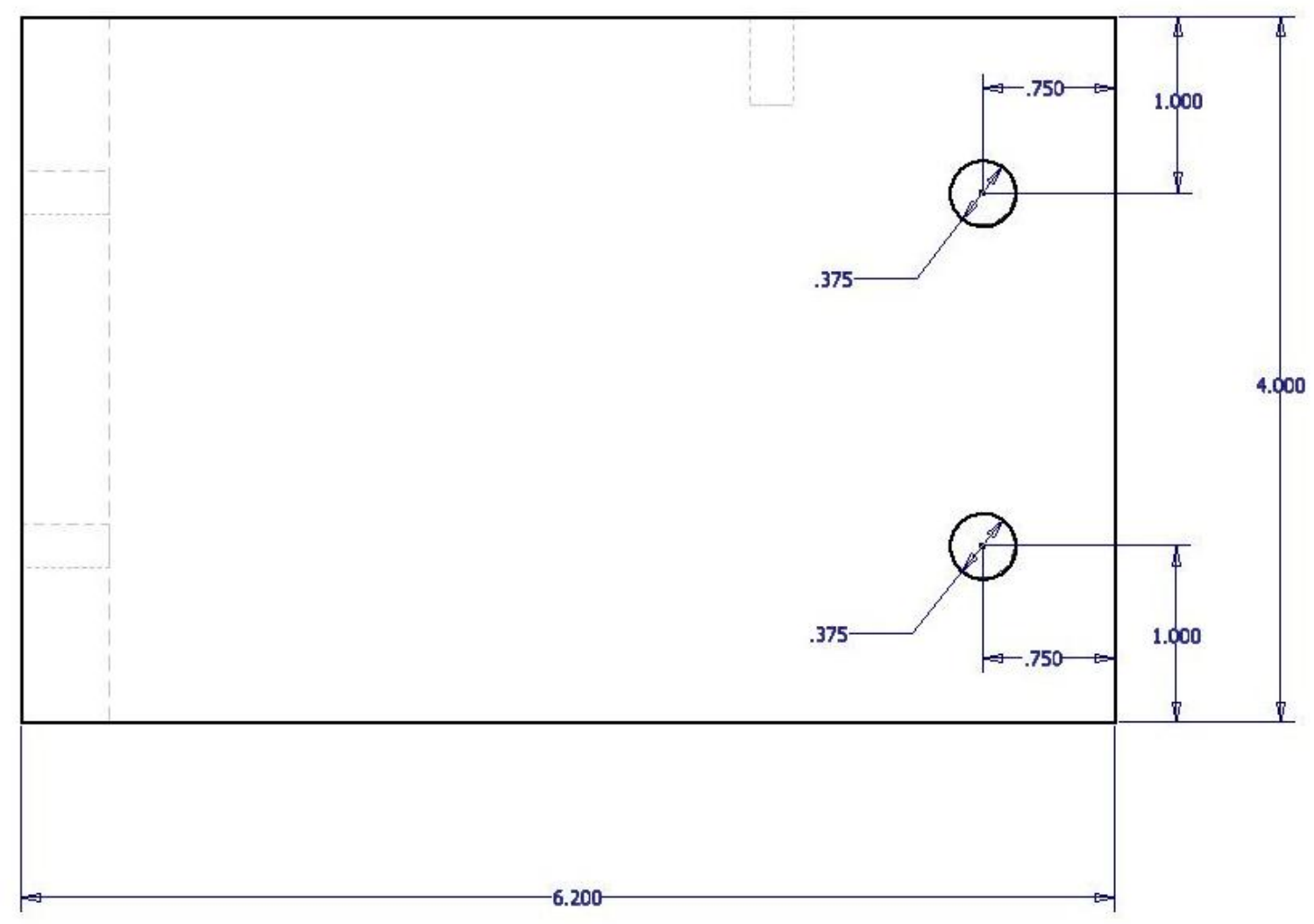

SIDE VIEW - ALUMINUM "L" PIECE 


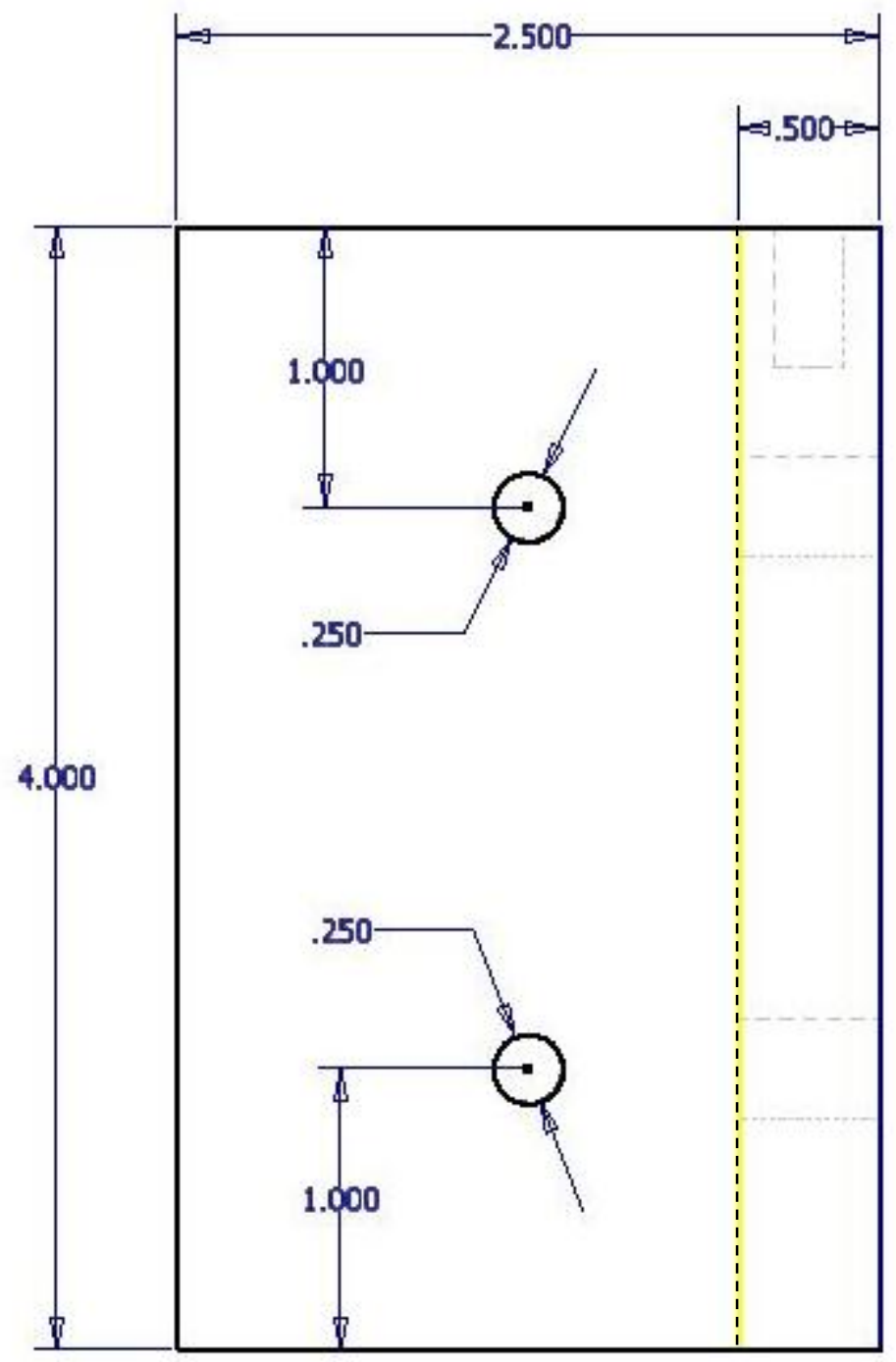

FRONT VIEW - LONG ALUMINUM SIDE ARM 


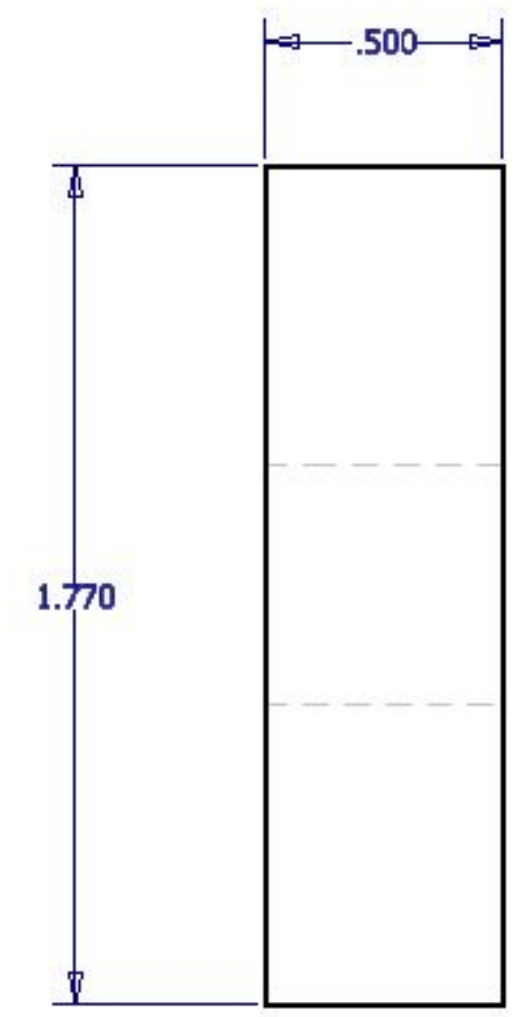

SIDE VIEW - LONG ALUMINUM SIDE ARM

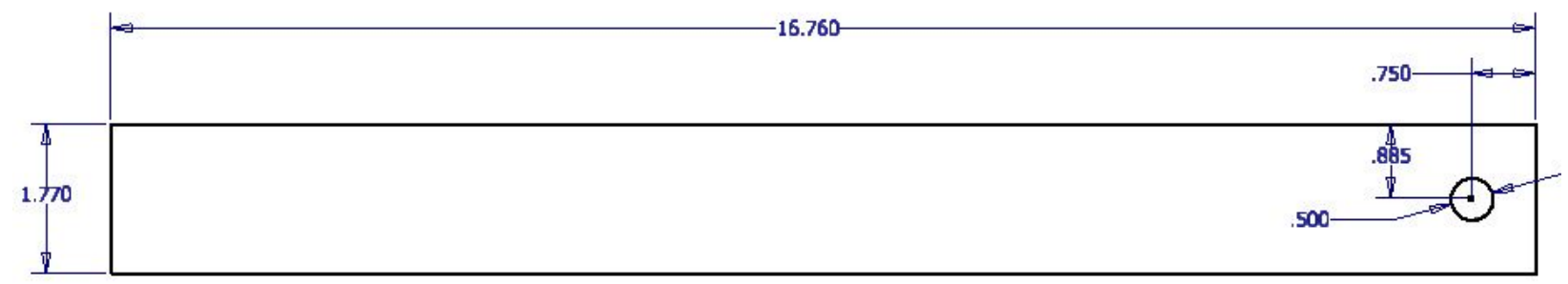

$\underline{\text { Lead brick weight subassembly }}$ 


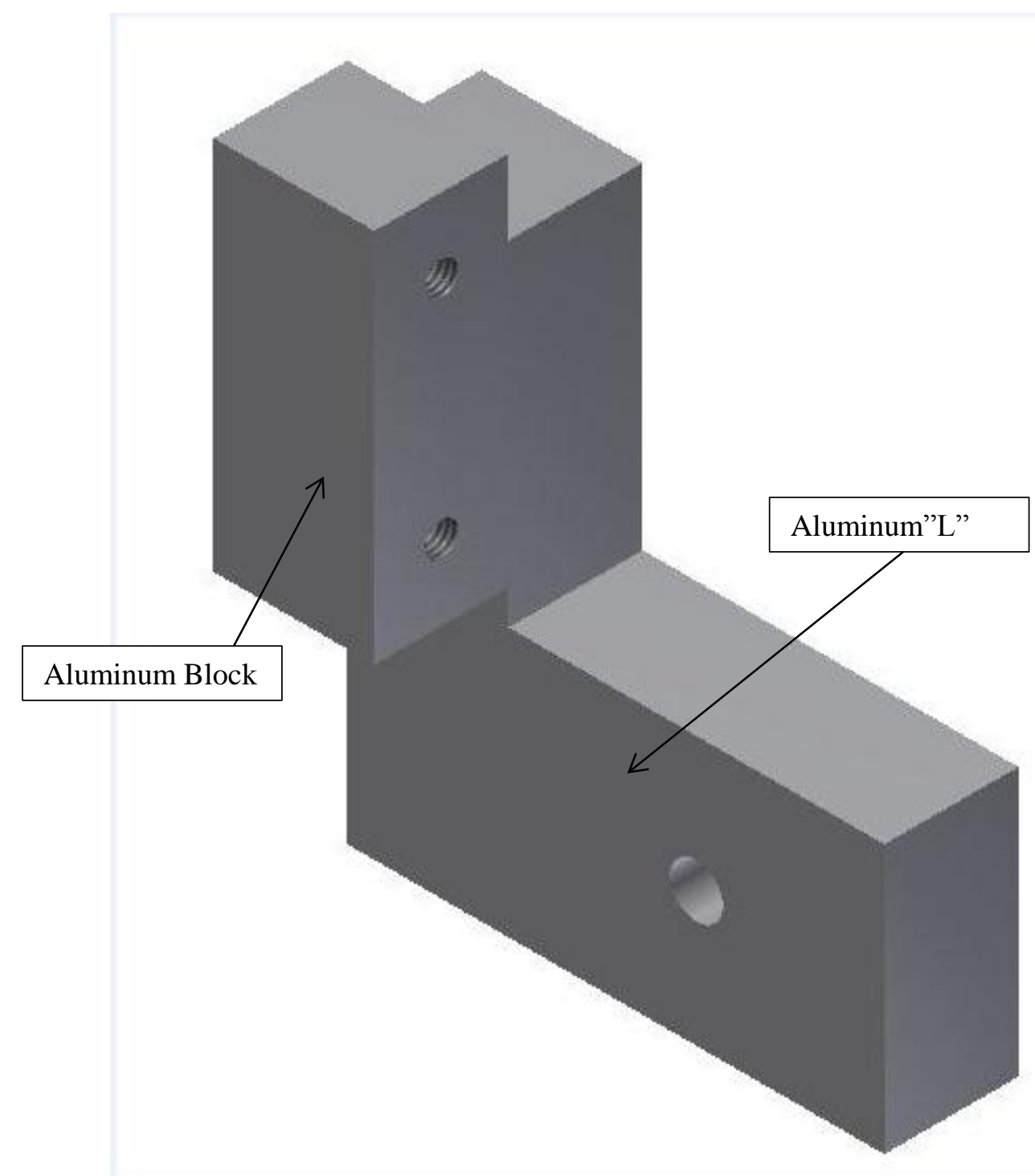


FRONT VIEW - ALUMINUM "L" PIECE

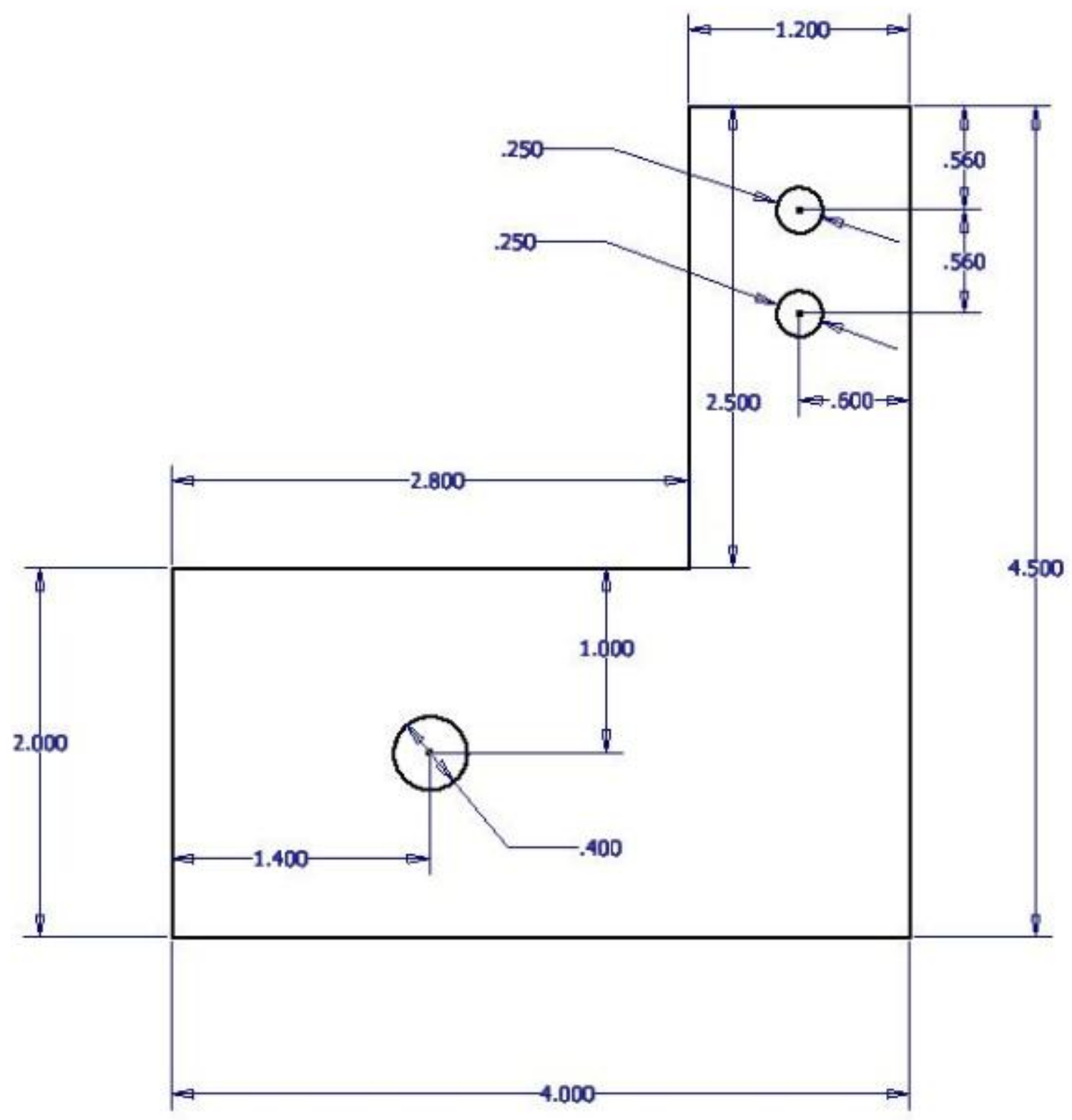




\section{SIDE VIEW - ALUMINUM "L" PIECE}

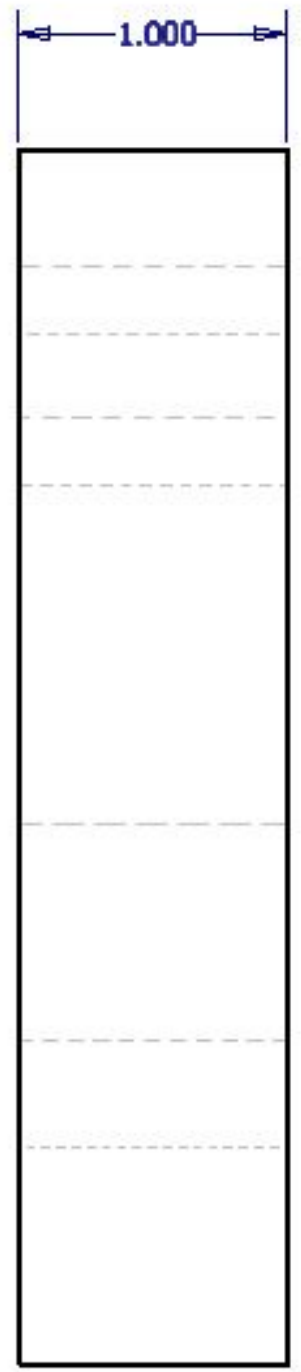




\section{FRONT VIEW - ALUMINUM BLOCK}

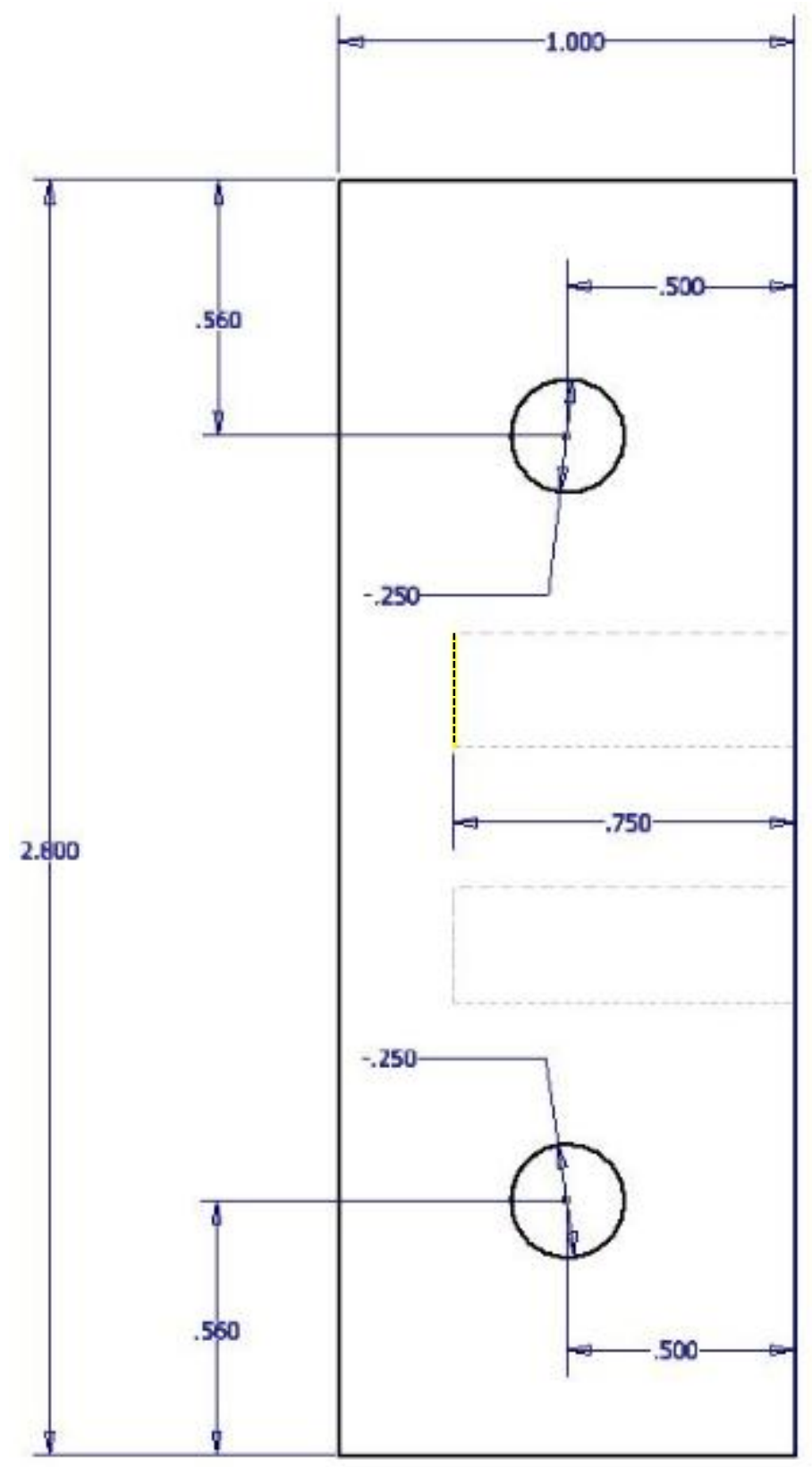




\section{SIDE VIEW - ALUMINUM BLOCK}

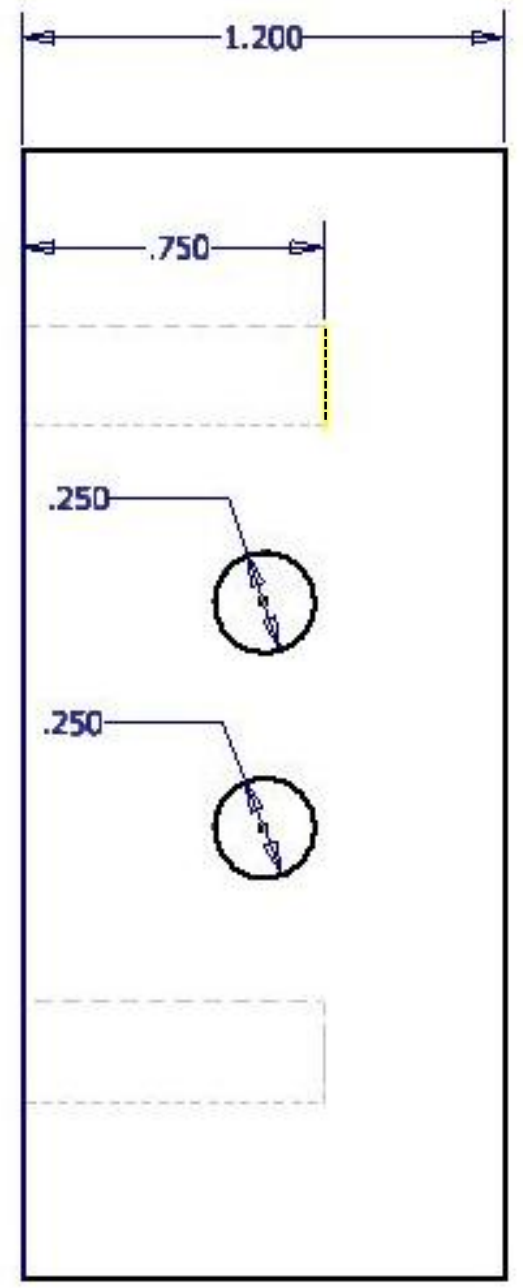




\section{References}

[1] Beaukbeuk (2009, March 13). Retrieved June 18, 2012 from http://en.wikipedia.org/wiki/File:Ameda_lactaline_personal_breast_pump.JPG\#file

[2] Blumenthal, W., Cady, C., Lopez, M., Gray, G., \& Idar, D. (2002). Influence of Temperature and Strain Rate on the Compressive Behavior of PMMA and Polycarbonate Polymers. American Institute of Physics, 620, 665-668. Retrieved from http://scitation.aip.org/getpdf/servlet/GetPDFServlet?filetype=pdf\&id=APCPCS0006200000010 $\underline{00665000001 \& \text { idtype }=\text { cvips } \& \text { doi }=10.1063 / 1.1483626 \& \text { prog }=\text { normal }}$

[3] Boas, G (2005, January). IMAGING TECHOLOGIES: Diffuse Optical Imaging. Retrieved from http://www.nmr.mgh.harvard.edu/martinos/research/technologiesDOI.php

[4] Boedeker Plastics (2012). Polycarbonate Specifications. Retrieved from http://www.boedeker.com/polyc_p.htm

[5] Breastcancer.org (2012). American College of Radiology Supports Starting Mammograms at 40. Retrieved from http://www.breastcancer.org/symptoms/testing/new_research/20100105.jsp?gclid=CJDJm_Wbq

\section{$\underline{\text { LACFQIN4AodxBuCXA }}$}

[6] Buitrago, Carlos (n.d.). Polycarbonate (PC). Retrieved from http://www.eng.buffalo.edu/Courses/ce435/PC_CB.pdf

[7] Cambridge University Engineering Dept (n.d.). Property Information: Young's Modulus and Specific Stiffness. Retrieved from http://www-materials.eng.cam.ac.uk/mpsite/properties/non$\underline{\text { IE/stiffness.html }}$

[8] Cedar-Sinai (2012). Breast MRI Procedure Information. Retrieved from http://www.cedarssinai.edu/Patients/Programs-and-Services/Imaging-Center/Imaging-Procedures/MRI/Breast/

[9] Cedar-Sinai (2012). Breast Ultrasound. Retrieved from http://www.cedarssinai.edu/Patients/Programs-and-Services/Imaging-Center/For-Patients/Exams-byProcedure/Ultrasound/Breast-Ultrasound/ 
[10] Centers for Disease Control and Prevention (CDC). Breast Cancer Statistics. Retrieved from http://www.cdc.gov/cancer/breast/statistics/

[11] Champaign, Judy L., \& Cederbom, Gunnar J. (2000, January). Advances in Breast Cancer Detection with Screening Mammography. Retrieved from http://www.ncbi.nlm.nih.gov/pmc/articles/PMC3117552/

[12] Dobbins, James T., \& Godfrey, Devon J. (2003, September 16). Digital X-ray Tomosynthesis:

Current State of the Art and Clinical Potential. Physics in Medicine and Biology, 48, R65-R106. Retrieved from http://iopscience.iop.org/0031-9155/48/19/R01

[13] Gong, Zongyi, Klanian, Kelly, Patel, Tushita, Sullivan, Olivia, \& Williams, Mark (Manuscript Submitted to Medical Physics for Publication). Implementation and Evaluation of an Expectation Maximization Reconstruction Algorithm for Gamma Emission Breast Tomosynthesis.

[14] Ideal Pharmacy (2012). Mammography: Patient Teaching Aid. Retrieved from http://www.idealpharmacyla.com/index.php?id=24

[15] Imaginis Corporation (2008). How Does Nuclear Medicine Work? Retrieved from http://www.imaginis.com/nuclear-medicine/how-does-nuclear-medicine-work

[16] Judy, Patricia G. (2010). Dual Modality Surgical Guidance. (Doctoral dissertation).

[17] Kuhl, Christiane K., Schrading, Simone, Leutner, Claudia C., Morakkabati-Spitz, Nuschin, Wardelmann, Eva, Fimmers, Rolf, Kuhn, Walther, \& Schild, Hans H. (2005, November 20). Mammography, Breast Ultrasound, and Magnetic Resonance Imaging for Surveillance of Women at High Familial Risk for Breast Cancer. Journal of Clinical Oncology, 23(33), 84698476. Retrieved from http://www.ncbi.nlm.nih.gov/pubmed/16293877

[18] Lee, Carol H., Dershaw, D., Kopans, Daniel, Evans, Phil, Monsees, Barbara, Monticciolo, Debra, Brenner, R., Bassett, Lawrence, Berg, Wendie, Feig, Stephen, Hendrick, Edward, Mendelson, Ellen, D'Orsi, Carl, Sickles, Edward, \& Burhenne, Linda (2010, January 2). Breast Cancer Screening With Imaging: Recommendations from the Society of Breast Imaging and the ACR on the Use of Mammography, Breast MRI, Breast Ultrasound, and Other Technologies for the 
Detection of Clinically Occult Breast Cancer. Journal of the American College of Radiology, 7(1), 18-27. Retrieved from

http://www.sciencedirect.com/science/article/pii/S1546144009004803

[19] Machinist-materials.com. Comparison Table for Plastics. Retrieved from http://www.machinist$\underline{\text { materials.com/comparison_table_for_plastics.htm }}$

[20] Mariani, John J. (2000). $2^{\text {nd }}$ Edition, Cracking the Boards: USMLE Step 2. Google Books, 91. Retrieved from http://books.google.com/books?id=NYAP52DQZsgC

[21] National Cancer Institute. SEER Training: Breast Anatomy. Retrieved from http://training.seer.cancer.gov/breast/anatomy/

[22] Pogue, Brian, Testorf, Markus, McBride, Troy, Osterberg, Ulf, \& Paulsen, Keith (1997, December 22). Instrumentation and Design of a Frequency-domain Diffuse Optical Tomography Imager for Breast Cancer Detection. Optics Express, 1(13), 391-403. Retrieved from http://www.opticsinfobase.org/view_article.cfm?gotourl=http $\% 3 \mathrm{~A} \% 2 \mathrm{~F} \% 2 \mathrm{Fwww} \% 2 \mathrm{Eopticsinfo}$ base\%2Eorg\%2FDirectPDFAccess\%2F7B51B16D\%2DC90F\%2DC461\%2D5C1CBFA9F3294 D00\%5F63220\%2Foe\%2D1\%2D13\%2D391\%2Epdf\%3Fda\%3D1\%26id\%3D63220\%26seq\%3 D0\%26mobile\%3Dno\&org=

[23] Poplack, Steven P., Tosteson, Tor D., Kogel, Christine A., \& Nagy, Helene M. (2007, April 19).

Digital Breast Tomosynthesis: Initial Experience in 98 Women with Abnormal Digital Screening Mammography. American Journal of Roentgenology, 189(3), 616-623. Retrieved from http://www.ajronline.org/content/189/3/616.short

[24] Schaefer, Jacob, Stejskal, E., Perchak, Dennis, Skolnick, Jeffery, \& Yaris, Robert (1995, March). Molecular Mechanism of the Ring-flip Process in Polycarbonate. Macromolecules (ACS Publications, 18(3), 368-373. Retrieved from http://pubs.acs.org/doi/abs/10.1021/ma00145a012

[25] Stanford University School of Medicine (2012). Patient Care: What is Nuclear Medicine? Retrieved from http://nuclearmedicine.stanford.edu/patient_care/

[26] Stony Brook University Physicians. Breast Cancer Center - Breast Lumps. Retrieved from http://www.stonybrookphysicians.com/center/breast-care-center-pi-breast-lumps.asp 
[27] Susan G. Komen for the Cure (2012). Breast Cancer Statistics. Retrieved from http://ww5.komen.org/BreastCancer/Statistics.html

[28] The Engineering Toolbox (n.d.). Elastic Properties and Young Modulus for Some Materials. Retrieved from http://www.engineeringtoolbox.com/young-modulus-d_417.html

[29] The Mathworks, Inc. (1994-2012). ANOVA1. Retrieved from http://www.mathworks.com/help/toolbox/stats/anova1.html

[30] The Mathworks, Inc. (1984-2012). Kruskalwallis. Retrieved from http://www.mathworks.com/help/toolbox/stats/kruskalwallis.html

[31] Virginia Industrial Plastics Inc. (n.d.). Retrieved from http://www.vaplastic.com/VacuumForming.html 